\title{
Computational Insight to Improve the Thermal Isomerisation Performance of Overcrowded Alkene-Based Molecular Motors through Structural Redesign
}

\author{
Baswanth Oruganti, Jun Wang and Bo Durbeej
}

\author{
Journal Article
}

\section{Tweet}

N.B.: When citing this work, cite the original article.

Original Publication:

Baswanth Oruganti, Jun Wang and Bo Durbeej, Computational Insight to Improve the Thermal Isomerisation Performance of Overcrowded Alkene-Based Molecular Motors through Structural Redesign, ChemPhysChem, 2016. 17(21), pp.3399-3408.

http://dx.doi.org/10.1002/cphc.201600766

Copyright: Wiley: 12 months

http://eu.wiley.com/WileyCDA/

Postprint available at: Linköping University Electronic Press

http://urn.kb.se/resolve?urn=urn:nbn:se:liu:diva-132609

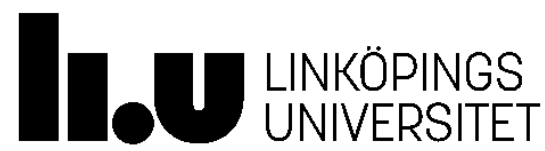




\section{Computational Insight to Improve the Thermal Isomerization Performance of Overcrowded Alkene-Based Molecular Motors through Structural Redesign}

Baswanth Oruganti, Jun Wang, and Bo Durbeej*[a]

[a] B. Oruganti, Dr. J. Wang, Prof. B. Durbeej

Division of Theoretical Chemistry, IFM

Linköping University

58183 Linköping (Sweden)

E-mail:bodur@ifm.liu.se

Supporting Information for this article can be found under http://dx.doi.org/x. 


\begin{abstract}
Synthetic overcrowded alkene-based molecular motors achieve $360^{\circ}$ unidirectional rotary motion of one motor half (rotator) relative to the other (stator) through sequential photochemical and thermal isomerization steps. In order to facilitate and expand the use of these motors for various applications, it is important to investigate ways to increase the rates and efficiencies of the reactions governing the rotary motion. Here, we use computational methods to explore whether the thermal isomerization performance of some of the fastest available motors of this type can be further improved by reducing the sizes of the motor halves. Presenting three new redesigned motors that combine an indanylidene rotator with a cyclohexadiene, pyran or thiopyran stator, we first use multiconfigurational quantum chemical methods to verify that the photoisomerizations of these motors sustain unidirectional rotary motion. Then, by performing density functional calculations, we identify both stepwise and concerted mechanisms for the thermal isomerizations of the motors and show that the rate-determining free-energy barriers of these processes are up to $25 \mathrm{~kJ} \mathrm{~mol}^{-1}$ smaller than those of the original motors. Furthermore, the thermal isomerizations of the redesigned motors proceed in fewer steps. Altogether, the results suggest that the redesigned motors are useful templates for improving the thermal isomerization performance of existing overcrowded alkene-based motors.
\end{abstract}




\section{Introduction}

The design and synthesis of molecules that can perform useful mechanical work by consuming energy is a major research field ${ }^{[1-4]}$ with potential applications in nanotechnology. ${ }^{[5-7]}$ Light is a clean and readily available source of energy for executing such work. Light-driven rotary molecular motors are molecules that exhibit repetitive unidirectional rotary motion about a carbon-carbon ${ }^{[8-24]}$ or carbon-nitrogen ${ }^{[25,26]}$ double bond through the absorption of UV or visible light. Motors of this type based on sterically overcrowded alkenes were first synthesized by Feringa and coworkers in the late nineties, ${ }^{[8,9]}$ and have subsequently attracted considerable interest. ${ }^{[10-23]}$ These motors contain two identical or distinct halves connected by a carbon-carbon double bond (axle) and achieve $360^{\circ}$ unidirectional rotation of one motor half (rotator) relative to the other half (stator) by means of consecutive photochemical and thermal isomerization steps.

An essential feature of overcrowded alkene-based molecular motors is the presence of at least one stereocenter, whose configuration $(R$ or $S)$ determines the direction of rotation (clockwise or counterclockwise). Another distinguishing chiral feature is the $P$ (right-handed) or $M$ (left-handed) helicity(ies) adopted by the motor half(ves) because of steric interactions between the rotator and stator in the so-called fjord regions (see Scheme 1). ${ }^{[8,9]}$ These interactions determine the rates of the thermal isomerizations, which are believed to limit the rotational frequencies that the motors can attain. ${ }^{[14,15,27,28]}$ Accordingly, a major experimental effort has been invested in optimizing the steric interactions between the motor halves in such a way that the thermal isomerizations are accelerated. ${ }^{[10-17,19-21]}$ Motors 1a-1c shown in Scheme 1, which have 
been found capable of achieving $\mathrm{MHz}$ rotational frequencies under suitable irradiation conditions $^{[15,19]}$ are key achievements of this work.

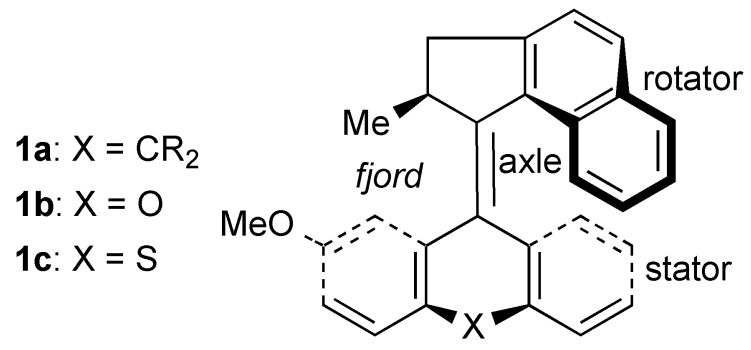

Scheme 1. Chemical structures of molecular motors 1a-1c.

Each $360^{\circ}$ rotation of motors of the type shown in Scheme 1 comprises two photoisomerizations and two thermal isomerizations that connect the $E$ and $Z$ isomers (with respect to the central olefinic bond) along the rotary cycle. This is illustrated in Scheme 2 for motor $\mathbf{1} \mathbf{c}$, which has a cyclopentanapthalynidene rotator and a thioxanthene stator. Each of the $E$ and $Z$ isomers of motor $1 \mathbf{c}$ has four possible conformations that differ in two ways. First, the stereogenic methyl substituent can adopt either a favorable pseudo-axial orientation, as in the "stable" isomers, or a strained (due to steric interactions) pseudo-equatorial orientation, as in the "unstable" isomers. Second, relative to the plane containing the central olefinic bond and the stereocenter (hereafter referred to as the olefinic plane), the rotator and stator can either fold toward the same side (synfolded) or toward opposite sides (anti-folded). In a recent computational study, the relative stabilities of these conformations and their roles in the rotary cycle of motor 1c were investigated by density functional theory (DFT) methods. ${ }^{[29]}$ 


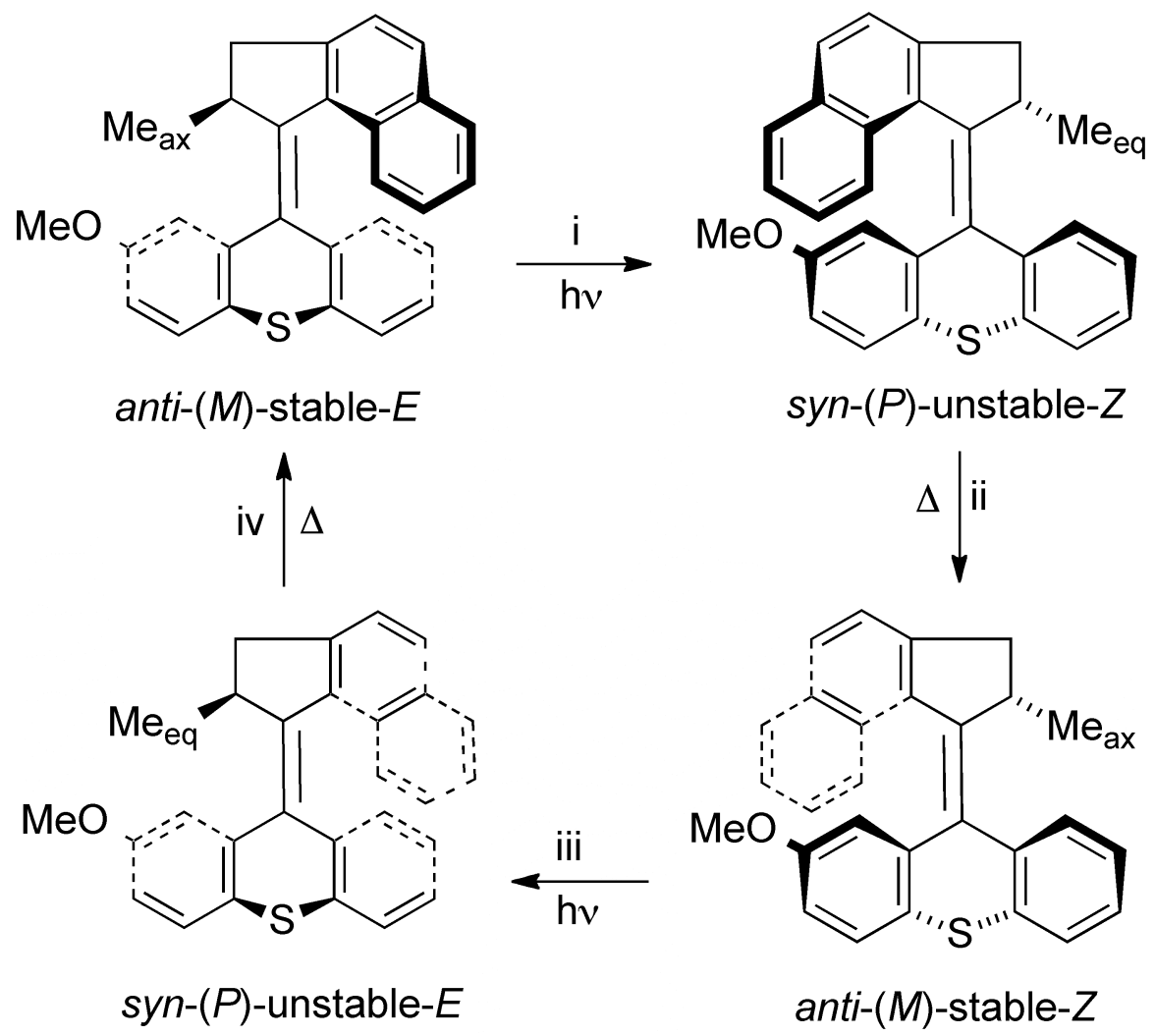

Scheme 2. Overall rotary cycle of molecular motor $\mathbf{1 c}$.

As shown in Scheme 2, the $E \rightarrow Z$ and $Z \rightarrow E$ photoisomerizations in the rotary cycle of motor 1c produce a strained unstable isomer, whereas the thermal isomerizations conversely release the strain and produce stable isomers. Further, each process involves a change in the rotator helicity $(M \rightarrow P$ or $P \rightarrow M)$ and a change in the relative rotatorstator folding (anti $\rightarrow$ syn or syn $\rightarrow$ anti). Overall, the rotary motion is governed by steric interactions between the rotator and stator in the fjord regions, which render the photoisomerizations unidirectional and the thermal isomerizations exergonic. Importantly, this exergonicity prevents photo-induced back rotations of the unstable isomers. $^{[9,14]}$ 
A key requirement for the possibility to efficiently use synthetic rotary molecular motors for applications (in, e.g., molecular transport ${ }^{[30]}$ or viscosity sensing,$^{[31,32]}$ ) is that they can achieve high rotational frequencies under ambient conditions. ${ }^{[14,33]}$ Therefore, many experimental studies have investigated how conformational, steric and electronic motor properties influence the rates of both the thermal isomerizations ${ }^{[10-17,19-21]}$ and photoisomerizations ${ }^{[27,28]}$ of overcrowded alkene-based motors. These studies have been complemented by several computational studies exploring the mechanisms of the rotary cycles of overcrowded alkenes, ${ }^{[21,29,34-42]}$ or proposing alternative light-driven motor designs with potentially more efficient rotary cycles. ${ }^{[43-47]}$

In our own computational work, we have established a three-step mechanism for the thermal isomerizations of motor $\mathbf{1 c}$ and several variants thereof, and also outlined systematic strategies for accelerating these processes by modulating the steric bulkiness of the rotator substituent. ${ }^{[29,42]}$ From a dynamical point of view, however, it would seem advantageous to develop motors whose thermal isomerizations occur in fewer than three steps. Furthermore, it would also be advantageous to eliminate the undesirable side reaction that was identified in both rotary half cycles of motor $\mathbf{1} \mathbf{c}$, and which is likely to impact the thermal isomerization rates negatively. ${ }^{[29]}$ In this paper, we present a computational study focused on these goals.

Specifically, we present a structural redesign of motors $\mathbf{1 a}-\mathbf{1 c}$, wherein the sizes of the rotator and stator are reduced to obtain motors $\mathbf{2 a - 2} \mathbf{c}$ shown in Scheme 3. In these motors, the cyclopentanapthalynidene rotator (3-ring system) of motors 1a-1c is replaced with a synthetically viable indanylidene $\operatorname{rotator}^{[16,24]}$ (2-ring system), and the phenanthrylidene, xanthene and thioxanthene stators (3-ring systems) of motors 1a-1c 
are replaced, respectively, with cyclohexadiene, pyran and thiopyran stators (1-ring systems).

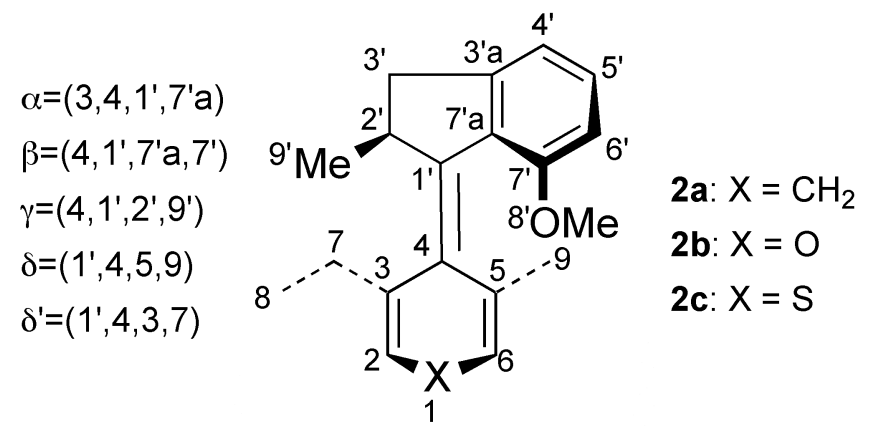

Scheme 3. Chemical structures, atom numbering and definition of dihedral angles in redesigned molecular motors $\mathbf{2 a}-\mathbf{2} \mathbf{c}$ considered in this work.

The paper is organized as follows. First, by performing multiconfigurational quantum chemical calculations, we show that the $E \rightarrow Z$ and $Z \rightarrow E$ photoisomerizations of motors $\mathbf{2 a}-\mathbf{2 c}$ occur in a unidirectional fashion and thus produce rotary motion. Then, by performing DFT calculations, we identify two possible mechanisms for the thermal isomerizations of these motors. Through the identified mechanisms, we find that the thermal isomerizations proceed in fewer steps than those of motor 1c and other overcrowded alkene-based motors, ${ }^{[21,41]}$ and furthermore have free-energy barriers that are up to $25 \mathrm{~kJ} \mathrm{~mol}^{-1}$ smaller. Furthermore, unlike motor 1c, the thermal isomerizations are not impeded by a side reaction. ${ }^{[29]}$ Thus, through this work, we propose that motors $\mathbf{2 a}-\mathbf{2 c}$ are members of a new family of overcrowded alkene-based rotary molecular motors capable of improving the thermal isomerization performance of some of the fastest motors of this type known to date (i.e., motors 1a-1c). 


\section{Computational Details}

The rotary cycles of motors $\mathbf{2 a - 2 c}$ were explored in the following way. First, the stationary points on the ground-state potential energy surfaces (PESs) corresponding to the stable and unstable $E$ and $Z$ isomers of the motors were located by performing geometry optimizations using the $\omega$ B97X-D range-separated hybrid density functional ${ }^{[48]}$ in combination with the SVP basis set. Frequency calculations were then performed at the same level of theory to ensure that the resulting geometries are potential-energy minima, and to derive Gibbs free energies at room temperature. Single point calculations, in turn, were carried out with $\omega$ B97X-D and Dunning's correlation-consistent polarized valence triple- $\zeta$ (cc-pVTZ) basis set. All calculations were performed with the $\mathrm{SMD}^{[49]}$ continuum solvation model to describe the dichloromethane solvent used in the experimental reference study of motor $1 \mathbf{c}^{[15]}$

Although it has been found that the calculated thermal barriers of motor 1c and several variants thereof are not very sensitive to the choice of density functional, ${ }^{[29]}$ there are at least three good reasons for using $\omega \mathrm{B} 97 \mathrm{X}-\mathrm{D}$ in the calculations. First, the merits of $\omega \mathrm{B} 97 \mathrm{X}-\mathrm{D}$ in organocatalytic modeling have been firmly established in an extensive benchmark study by Clark and coworkers. ${ }^{[50]}$ Second, it has been found that $\omega$ B97X-D yields thermal free-energy barriers for reference motor $1 \mathbf{c}^{[29]}$ that are in good agreement with available experimental data. ${ }^{[15]}$ Third, because of its inclusion of empirical atomatom dispersion corrections, ${ }^{[51,52]} \omega \mathrm{B} 97 \mathrm{X}-\mathrm{D}$ is well-suited to describe the critical intramolecular interactions between the motor halves during the rotary cycles.

From the calculations on the stable and unstable $E$ and $Z$ isomers of motors $2 \mathbf{2 a}-$ $\mathbf{2 c}$, it was observed that, for each of the three motors, the two lowest-energy isomers are 
anti-(M)-stable- $E$ and anti-( $(M)$-stable-Z. As further discussed in section 2.1 below, these isomers show a sterically favorable anti folding of the rotator and stator substituents relative to the olefinic plane. Also, these are the light-absorbing isomers of the rotary cycles.

As the main focus of this work is to offer guidelines for improving the thermal isomerization performance of overcrowded alkene-based motors, the photochemical modeling of motors $\mathbf{2 a}-\mathbf{2} \mathbf{c}$ was limited to demonstrating the unidirectionality of the $E \rightarrow$ $Z$ and $Z \rightarrow E$ photoisomerizations of the light-absorbing isomers. This was done by performing multiconfigurational quantum chemical calculations using the complete active space self-consistent field (CASSCF) ${ }^{[53]}$ and second-order perturbation theory (CASPT2 ${ }^{[54,55]}$ methods, as further described in the Supplementary Computational Details section of the Supporting Information. Furthermore, from these calculations, it was also established that the $E \rightarrow Z$ photoisomerization of anti-(M)-stable- $E$ produces the $(P)$-unstable- $Z$ ground-state isomer, and that the $Z \rightarrow E$ photoisomerization of anti-(M)stable- $Z$, analogously, yields the $(P)$-unstable- $E$ ground-state isomer. Notably, these photoisomerization products lack the type of rotator and stator folding manifested in the parent light-absorbing isomers. Below, we will return to this issue and its mechanistic implications.

Having found the $E \rightarrow Z$ and $Z \rightarrow E$ photoisomerization products of motors 2a2c, the mechanisms for the subsequent thermal $(P)$-unstable- $Z \rightarrow$ anti- $(M)$-stable- $Z$ and $(P)$-unstable- $E \rightarrow$ anti-( $(M)$-stable- $E$ isomerizations that complete the rotary cycles were explored by locating all the relevant transition structures (TSs) and intermediates through $\omega \mathrm{B} 97 \mathrm{X}-\mathrm{D}$ calculations as detailed above. The corresponding frequency calculations 
showed that all the resulting stationary points have either zero (for minima) or one (for TSs) imaginary vibrational frequency. In addition, intrinsic reaction coordinate (IRC) ${ }^{[56]}$ calculations were carried out to verify that the TSs do indeed mediate relevant chemical transformations.

All DFT calculations were performed with the Gaussian 09 suite of programs, ${ }^{[57]}$ and all CASSCF and CASPT2 calculations were performed with the MOLCAS 8.0 suite of programs. ${ }^{[58]}$

\section{Results and Discussion}

\subsection{Ground-State Minima of Motors 2a-2c}

Each ground-state minimum $(E$ or $Z$ ) of motors $\mathbf{2 a}-\mathbf{2} \mathbf{c}$ was found to have three possible conformations that differ in two ways. First, analogous to the situation for motor $\mathbf{1} \mathbf{c}^{[29]}$ the rotator methyl substituent can either adopt a pseudo-axial ("stable") or a pseudoequatorial ("unstable") orientation. Second, as illustrated in Scheme 4, the stable isomers have two different conformations with respect to the orientation of the rotator and stator substituents relative to the olefinic plane. In the syn-folded conformations, these substituents are oriented toward the same side of the olefinic plane, whereas they are oriented toward opposite sides in the anti-folded conformations. As a result, the antifolded conformations exhibit less steric overcrowding in the fjord regions than the synfolded conformations. For the unstable isomers, such folding is not manifested because the stator substituents are nearly co-planar with the central olefinic bond. This is a key structural feature that distinguishes motors $\mathbf{2 a - 2} \mathbf{c}$ from reference motor $\mathbf{1} \mathbf{c}^{[29]}$ and other overcrowded alkene-based motors, ${ }^{[21,59]}$ whose unstable isomers can be both anti-folded 
and syn-folded. As we will see, this feature has a significant impact on the mechanisms for the thermal isomerizations of motors $\mathbf{2 a}-\mathbf{2} \mathbf{c}$.
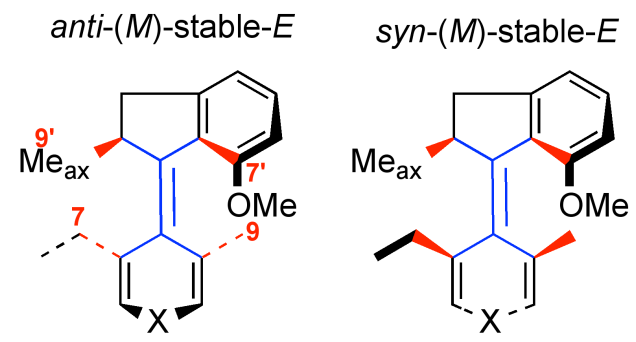

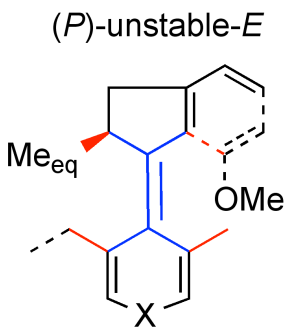

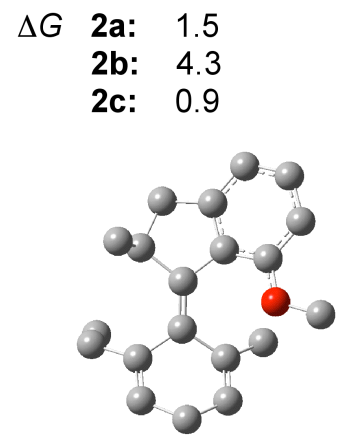

25.1

16.9

30.6

$$
\begin{array}{ll}
25.2 & \mathrm{X}=\mathrm{CH}_{2} \\
17.2 & \mathrm{X}=\mathrm{O} \\
37.5 & \mathrm{X}=\mathrm{S}
\end{array}
$$
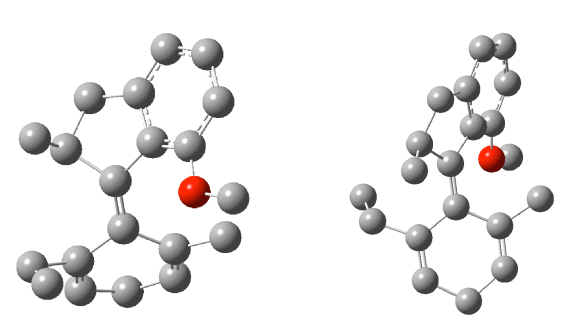

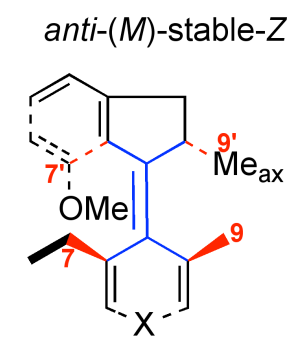

syn-(M)-stable-Z

(P)-unstable-Z<smiles>C=c1c(C)ccc(C)c1=C1c2c(cccc2OC)CC1C</smiles><smiles></smiles>

$\Delta G$ 2a: 0.0

2b: 0.0

2c: 0.0

$$
\begin{aligned}
& 31.8 \\
& 28.7 \\
& 27.8
\end{aligned}
$$
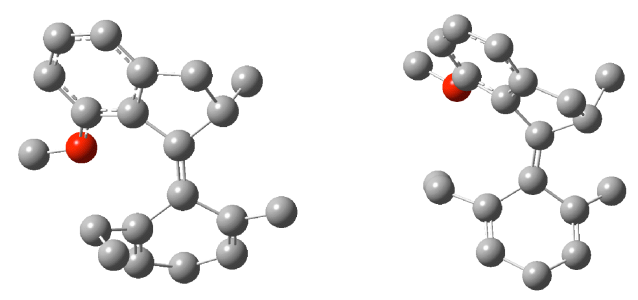

Scheme 4. Structures of the ground-state minima of motors $\mathbf{2 a - 2 c}$ and their relative $\omega \mathrm{B} 97 \mathrm{X}-\mathrm{D}$ free energies $\left(\Delta G\right.$, in $\left.\mathrm{kJ} \mathrm{mol}^{-1}\right)$. Shown also are the corresponding optimized structures of motor $\mathbf{2 a}$ (without hydrogen atoms). 
Scheme 4 also shows the relative $\omega \mathrm{B} 97 \mathrm{X}-\mathrm{D}$ free energies of the ground-state minima of motors $\mathbf{2 a - 2 c}$. As can be seen, the parent light-absorbing anti-(M)-stable- $E$ and anti- $(M)$-stable- $Z$ species are by some margin the most stable isomers. Comparing their energies with those of the $(P)$-unstable- $Z$ and $(P)$-unstable- $E$ isomers produced (see Section 2.2) by the $E \rightarrow Z$ and $Z \rightarrow E$ photoisomerizations, these energy differences vary considerably between the motors; from $15-17 \mathrm{~kJ} \mathrm{~mol}^{-1}$ for motor $\mathbf{2 b}$ (with $\mathrm{X}=\mathrm{O}$ ) to $\sim 25$ and $\sim 38 \mathrm{~kJ} \mathrm{~mol}^{-1}$ for motors $\mathbf{2 a}\left(\mathrm{X}=\mathrm{CH}_{2}\right)$ and $\mathbf{2 c}(\mathrm{X}=\mathrm{S})$, respectively. This is because the increase in the size of stator bridging atom $\mathrm{X}$ from oxygen in motor $\mathbf{2 b}$ to sulphur in motor $2 \mathbf{c}$ increases the steric repulsion in the fjord regions of the photoisomerization products. Thus, these results, which are corroborated by complementary CASPT2//CASSCF energies in Table S1 of the Supporting Information, suggest that the stator bridging atom is a suitable element for exerting control of the rotary cycle.

\subsection{Unidirectionality and Products of the Photoisomerizations of Motors 2a-2c}

Although this work focuses on improving the thermal isomerization performance of overcrowded alkene-based motors through structural redesign of existing motors, it is important to first verify that the $E \rightarrow Z$ and $Z \rightarrow E$ photoisomerizations of motor candidates $2 \mathbf{2}-\mathbf{2 c}$ actually produce unidirectional rotary motion. This was done by modeling, using CASSCF and CASPT2 as described in the Supplementary Computational Details section of the Supporting Information, the geometric relaxation of the light-absorbing anti-(M)-stable- $E$ and anti-(M)-stable- $Z$ isomers from the vertically excited Franck-Condon (FC) points in the lowest excited singlet state $\left(\mathrm{S}_{1}\right)$ populated by 
light absorption. Producing $\mathrm{S}_{1}$ minima henceforth labeled anti-(M)-stable- $E^{*}$ and anti$(M)$-stable- $Z^{*}$, the results of these calculations are presented in Figure 1.
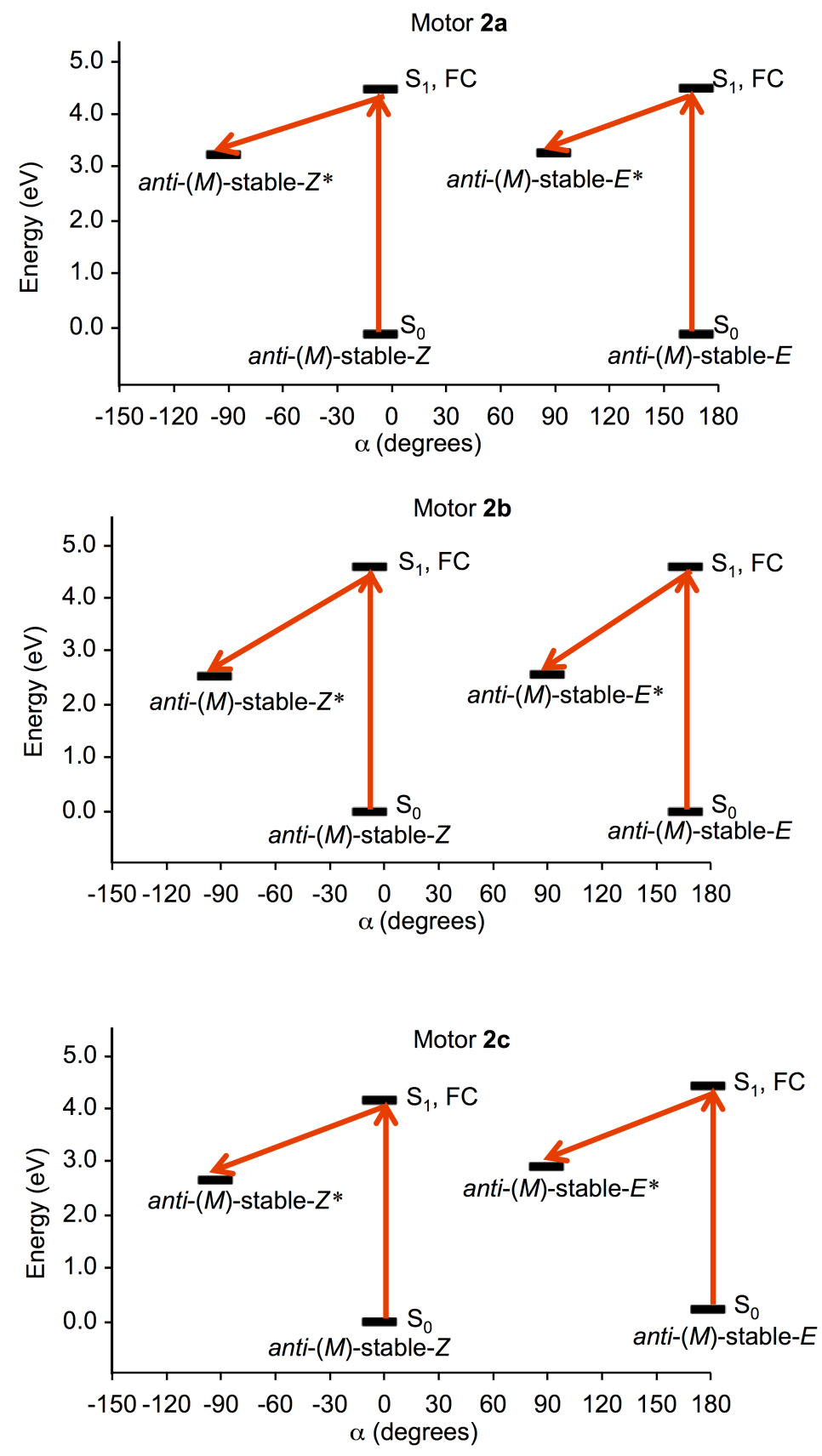

Figure 1. FC relaxation of the light-absorbing anti-( $(M)$-stable- $E$ and anti-( $(M)$-stable- $Z$ isomers of motors $\mathbf{2 a}-\mathbf{2} \mathbf{c}$. 
Notably, Figure 1 shows that the FC relaxation processes of all three motors involve appreciable torsional motion along the $\alpha$ photoisomerization coordinate (see Scheme 3). Specifically, the torsional motion amounts to $76-83^{\circ}$ for the $\mathrm{FC}$ relaxation of the anti-(M)-stable- $E$ isomers, and to $84-91^{\circ}$ for the FC relaxation of the anti-(M)-stable$Z$ isomers. This motion is facilitated by the concurrent elongation of the central olefinic bond by $0.12-0.14 \AA$ in the excited state, details of which are given in Table S2 of the Supporting Information. More importantly, however, for each of the three motors, the direction of photoinduced torsional motion is the same - toward decreasing $\alpha$ - for the anti-(M)-stable- $E$ and anti-( $(M)$-stable- $Z$ isomers. Thus, the associated $E \rightarrow Z$ and $Z \rightarrow E$ photoisomerizations occur in a unidirectional fashion and produce rotary motion.

From previous computational studies of overcrowded alkene-based motors, the formation of the ground-state $\left(\mathrm{S}_{0}\right)$ photoisomerization products is likely to be mediated by $\mathrm{S}_{1} / \mathrm{S}_{0}$ conical intersections at highly twisted molecular geometries along the $\alpha$ coordinate. ${ }^{[36,37]}$ Here, rather than firstly trying to locate such intersections, the photoproducts were located directly by performing CASSCF $\mathrm{S}_{0}$ geometry optimizations starting from the (already highly twisted) anti-(M)-stable- $E^{*}$ and anti-(M)-stable- $Z^{*} \mathrm{~S}_{1}$

minima. These calculations yielded $(P)$-unstable- $Z$ as the photoproduct of the $E \rightarrow Z$ isomerization and $(P)$-unstable- $E$ as the photoproduct of the $Z \rightarrow E$ isomerization. Thus, the photoisomerizations invert the rotator helicity from $M$ to $P$.

\subsection{The Thermal Isomerizations of Motors 2a-2c}

In the $(P)$-unstable- $Z$ and $(P)$-unstable- $E$ photoproducts of motors $\mathbf{2 a - 2}$, the stereogenic rotator methyl substituent adopts a strained pseudo-equatorial orientation because of 
steric interactions with the stator. During the subsequent thermal $(P)$-unstable- $Z \rightarrow$ anti$(M)$-stable- $Z$ and $(P)$-unstable- $E \rightarrow$ anti- $(M)$-stable- $E$ isomerizations that complete the rotary cycles, the methyl regains its preferred pseudo-axial orientation as the rotator helicity changes from $P$ to $M$. As a result, the thermal isomerizations are exergonic, which introduces a "forward" driving force in the rotary cycles and depletes the photoproducts, thereby preventing undesirable photo-induced back reactions. ${ }^{[9,14]}$

Two possible mechanisms were identified for the thermal isomerizations, one being concerted and occurring without any intermediate(s), and the other occurring through two separate steps. The calculated free-energy profiles for these mechanisms are presented in Figure 2. Associated changes in the $\beta, \gamma, \delta$ and $\delta^{\prime}$ (see Scheme 3) dihedral angles, which respectively reflect rotator helicity and orientations of the rotator methyl, stator methyl, and stator ethyl group, are given in Tables S3-S5 of the Supporting Information. Figure 3, finally, shows optimized TSs for the case of motor 2a. 
$(P)$-unstable- $Z \rightarrow$ anti- $(M)$-stable- $Z \quad(P)$-unstable- $E \rightarrow$ anti-(M)-stable- $E$
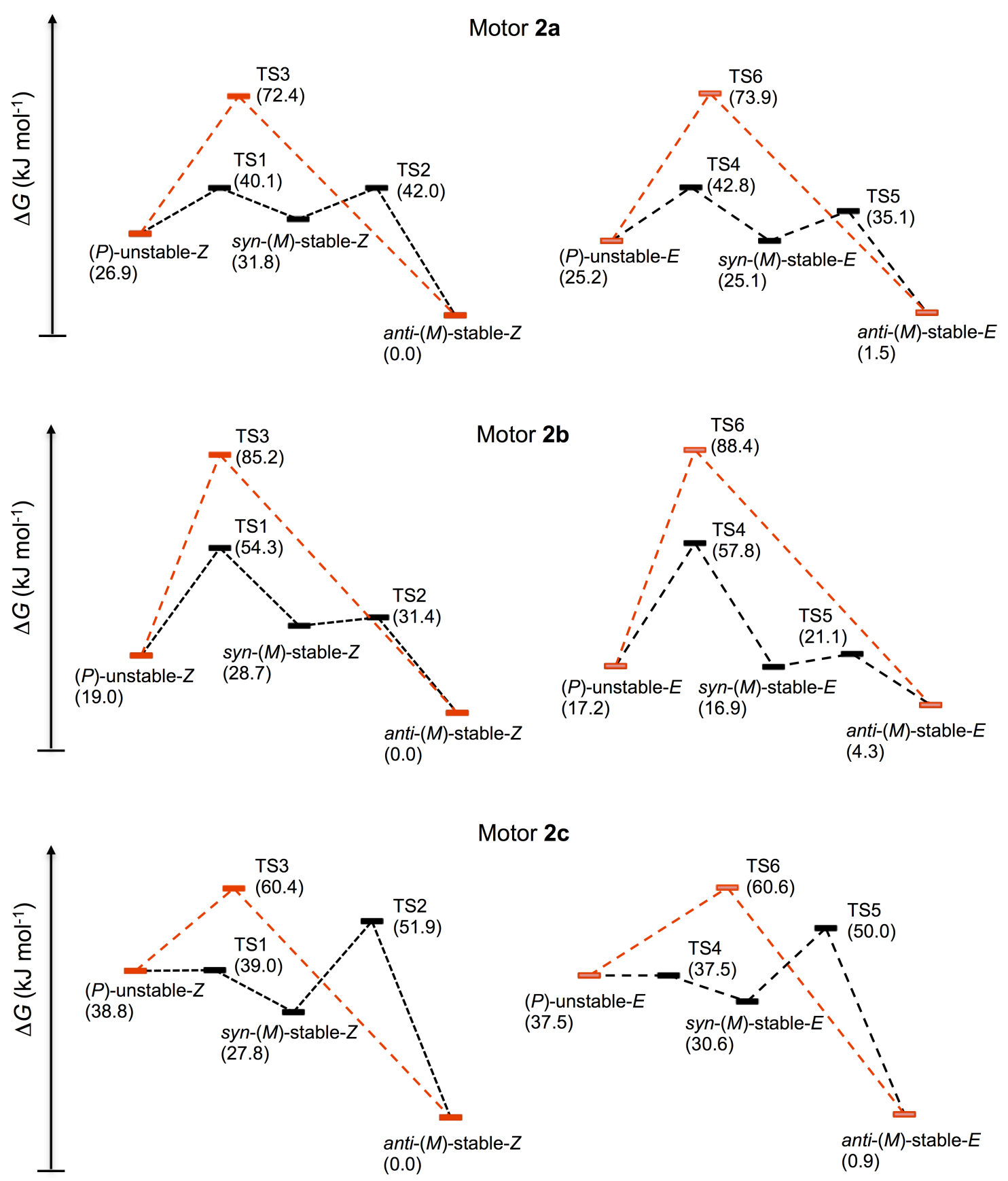

Figure 2. Free-energy profiles for stepwise and concerted mechanisms for the thermal isomerizations of motors $\mathbf{2 a}-\mathbf{2} \mathbf{c}$. 


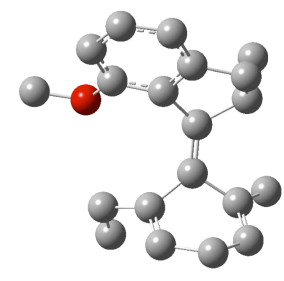

TS1

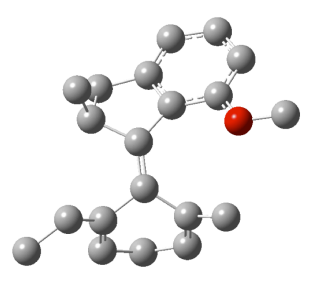

TS4

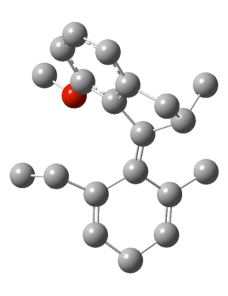

TS2

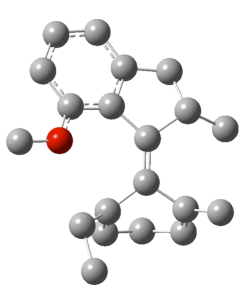

TS3

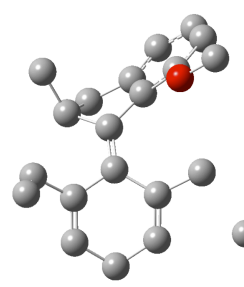

TS5

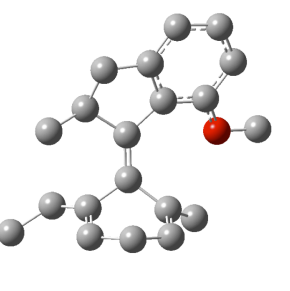

TS6

Figure 3. Optimized TSs for the thermal isomerizations of motor 2a (hydrogen atoms not shown).

Starting with the stepwise mechanism, for each of the motors, the TS for the first step is denoted TS1 for the isomerization of $(P)$-unstable- $Z$ and TS4 for the isomerization of $(P)$-unstable- $E$. This step involves a $P \rightarrow M$ change in rotator helicity that releases the conformational strain of the pseudo-equatorial stereogenic methyl in the initial species. Furthermore, while the rotator and stator substituents are neither syn- nor anti-folded in the initial species, the intermediates produced by this step are $s y n$-folded: $s y n$ - $(M)$-stable$Z$ and syn-(M)-stable-E. As can be seen from Figure 2, the TS1/TS4 free-energy barriers increase with decreasing size of the stator bridging atom $\mathrm{X}$ of the motor; from virtually zero for motor $\mathbf{2 c}(\mathrm{X}=\mathrm{S})$ to $13-18$ and $35-41 \mathrm{~kJ} \mathrm{~mol}^{-1}$ for motors $\mathbf{2 a}\left(\mathrm{X}=\mathrm{CH}_{2}\right)$ and $\mathbf{2 b}$ $(\mathrm{X}=\mathrm{O})$, respectively. Qualitatively, this can be understood by noting, in Table S6 of the Supporting Information, that the appreciable fjord-region steric repulsion in the $(P)$ unstable- $Z$ and $(P)$-unstable- $E$ reactant isomers of motor $\mathbf{2 c}$, is much reduced in TS1/TS4 
of this motor. Thereby, decreasing the size of the bridging atom from sulphur (motor $\mathbf{2 c}$ ) to oxygen (motor $\mathbf{2 b}$ ) has a stabilizing effect on $(P)$-unstable- $Z$ and $(P)$-unstable- $E$, which is not fully countered by a corresponding effect on TS1/TS4.

Continuing with the second step that proceeds via TS2/TS5 along the Z/E pathway, this step completes the thermal isomerizations by accomplishing a stator ring flip that changes the relative rotator-stator folding from syn in the intermediates to anti in the final anti-( $M)$-stable- $Z$ and anti- $(M)$-stable- $E$ isomers. By light absorption of these isomers, the rotary cycles are then continued photochemically. Notably, the TS2/TS5 barriers show a contrasting trend to the TS1/TS4 barriers; decreasing from 19-24 $\mathrm{kJ} \mathrm{mol}^{-}$ ${ }^{1}$ for motor $\mathbf{2 c}$ to 10 and $3-4 \mathrm{~kJ} \mathrm{~mol}^{-1}$ for motors $\mathbf{2 a}$ and $\mathbf{2 b}$, respectively. This, too, can be understood in terms of how the steric repulsion in motor $\mathbf{2} \mathbf{c}$ is different in the TSs than in the associated reactant species.

Overall, the rate-determining step of the stepwise mechanism is the first step for motors 2a $\left(\Delta G^{\ddagger}=13-18 \mathrm{~kJ} \mathrm{~mol}^{-1}\right)$ and $\mathbf{2 b}\left(\Delta G^{\ddagger}=35-41 \mathrm{~kJ} \mathrm{~mol}^{-1}\right)$, and the second step for motor $\mathbf{2 c}\left(\Delta G^{\ddagger}=19-24 \mathrm{~kJ} \mathrm{~mol}^{-1}\right)$. For motors $2 \mathbf{a}$ and $\mathbf{2 b}$, one potential caveat of this mechanism is the slight $\left(5-10 \mathrm{~kJ} \mathrm{~mol}^{-1}\right)$ endergonicity of the first step of the isomerization of $(P)$-unstable- $Z$, which means that the reverse isomerization of the syn$(M)$-stable- $Z$ intermediate back to $(P)$-unstable- $Z$ is predicted to have a smaller barrier than the forward reaction. However, the negative impact of this scenario seems to be nullified by the $29-32 \mathrm{~kJ} \mathrm{~mol}^{-1}$ exergonicity of the subsequent second step.

Turning to the concerted mechanism, for each of the motors, the corresponding TS is denoted TS3 for the isomerization of $(P)$-unstable- $Z$ and TS6 for the isomerization of $(P)$-unstable- $E$. In these TSs, the $P$ rotator helicity and the strained pseudo-equatorial 
orientation of the stereogenic methyl shown by the initial $(P)$-unstable- $Z$ and $(P)$ unstable- $E$ species are retained, contrary to the situation in TS1/TS4 of the stepwise mechanism. In this regard, TS3 and TS6 can be described as being highly asynchronous. This description is reminiscent of the way that organic pericyclic reactions oftentimes occur in a perfectly concerted fashion (i.e., without intermediates), despite that the corresponding TSs are asynchronous with respect to how far the bond formation processes mediated by the TSs have progressed. ${ }^{[60-62]}$ Notwithstanding, in TS3/TS6, the stator substituents are no longer co-planar with the olefinic bond, as they are in the initial species (e.g., for motor $\mathbf{2 a}$, the $\delta$ dihedral angle changes from $9^{\circ}$ in $(P)$-unstable- $Z$ to $75^{\circ}$ in TS3, see Table S3). This facilitates the $P \rightarrow M$ change in rotator helicity that releases the stereogenic methyl strain and, without any further barrier, completes the isomerizations by producing the anti- $(M)$-stable- $Z$ and anti-( $M)$-stable- $E$ isomers. Comparing the different motors, the TS3/TS6 barriers increase from $22-23 \mathrm{~kJ} \mathrm{~mol}^{-1}$ for motor $2 \mathbf{c}$ to $46-49$ and $66-71 \mathrm{~kJ} \mathrm{~mol}^{-1}$ for motors $\mathbf{2 a}$ and $\mathbf{2 b}$, respectively. The overall exergonicities of the isomerizations, in turn, decrease from $37-39 \mathrm{~kJ} \mathrm{~mol}^{-1}$ for motor $\mathbf{2 c}$ to $24-27$ and $13-19 \mathrm{~kJ} \mathrm{~mol}^{-1}$ for motors $\mathbf{2 a}$ and $\mathbf{2 b}$, respectively. This can be explained in terms of how the different sizes of the stator bridging atom of the motors introduce different amounts of steric repulsion in the $(P)$-unstable- $Z$ and $(P)$-unstable- $E$ reactants.

Comparing the calculated free-energy barriers of the stepwise and concerted mechanisms, the stepwise mechanism has a rate-determining barrier that - for motors 2a and $\mathbf{2 b}-$ is $\sim 30 \mathrm{~kJ} \mathrm{~mol}^{-1}$ smaller than the concerted barrier. For motor $\mathbf{2} \mathbf{c}$, an analogous comparison reveals that the two mechanisms are equally favorable in this respect. As explained above, a key difference between the mechanisms is that the conformational 
strain of the stereogenic methyl is already released in TS1/TS4 (stepwise), which is not the case in TS3/TS6 (concerted). For example, using motor $\mathbf{2 a}$ to illustrate this, Table S3 shows that the $\gamma$ dihedral angle changes from $40^{\circ}$ in $(P)$-unstable- $Z$ to $112^{\circ}$ in TS1, but only to $24^{\circ}$ in TS3. This difference may explain why the stepwise mechanism is preferred over the concerted ditto, at least as far as motors $\mathbf{2} \mathbf{a}$ and $\mathbf{2 b}$ are concerned.

Overall, for motors $\mathbf{2 a}$ and $\mathbf{2} \mathbf{b}$, the rate-determining barriers of the preferred stepwise mechanism are $18(\mathbf{2 a})$ and $41 \mathrm{~kJ} \mathrm{~mol}^{-1}(\mathbf{2 b})$. For motor $\mathbf{2 c}$, in turn, the ratedetermining barrier of the stepwise mechanism is $24 \mathrm{~kJ} \mathrm{~mol}^{-1}$, which is very similar to the barrier of $23 \mathrm{~kJ} \mathrm{~mol}^{-1}$ for the concerted process. Comparing these values with the calculated - using the same exact methodology - rate-determining barrier of $43 \mathrm{~kJ} \mathrm{~mol}^{-1}$ for reference motor $\mathbf{1 c},{ }^{[29]}$ which is close to the corresponding experimental estimate, ${ }^{[15]}$ no rate acceleration is observed for motor $\mathbf{2 b}$. For motors $\mathbf{2 a}$ and $\mathbf{2 c}$, on the other hand, the thermal isomerizations are kinetically favored by 25 and $19-20 \mathrm{~kJ} \mathrm{~mol}^{-1}$, respectively. From a dynamical point of view, it is also encouraging that the thermal isomerizations of motors $\mathbf{2 a}-\mathbf{2} \mathbf{c}$ proceed in fewer steps than those of motor $\mathbf{1} \mathbf{c}^{[29]}$ and other overcrowded alkene-based motors. ${ }^{[21,41]}$ Furthermore, as a consequence of the absence of syn- or antifolded conformations for the photoproduct isomers of motors $\mathbf{2 a - 2}$, there are no syn $\rightarrow$ anti equilibration side reactions to hamper the performance of these motors, which is a key advantage over motor $\mathbf{1} \mathbf{c}^{[29]}$ and related motors. ${ }^{[21,59]}$ Altogether, based on the results of this study, we propose that motors $\mathbf{2 a} \mathbf{a}-\mathbf{2}$ constitute a new family of overcrowded alkene-based motors with the potential to improve the thermal isomerization performance of existing motors of this type. 
Finally, to solidify this proposal, the thermal isomerizations of motors $\mathbf{2 a}-\mathbf{2 c}$ were also explored using the M06-2X global hybrid density functional, ${ }^{[63]}$ as a complement to the $\omega \mathrm{B} 97 \mathrm{X}-\mathrm{D}$ calculations. All M06-2X calculations were performed in the same way as the $\omega \mathrm{B} 97 \mathrm{X}-\mathrm{D}$ ones with respect to choice of basis set and treatment of solvent effects, as already described in the Computational Details section. The resulting free-energy profiles for both the stepwise and concerted mechanisms are shown in Figure S2 of the Supporting Information. Pleasingly, these profiles support the same mechanistic features as the $\omega$ B97X-D calculations summarized in Figure 2. For example, for motors 2a and $\mathbf{2 b}$, the stepwise mechanism is favored over the concerted one by $29-31 \mathrm{~kJ} \mathrm{~mol}^{-1}$, which is close to the value of $31 \mathrm{~kJ} \mathrm{~mol}^{-1}$ predicted by $\omega \mathrm{B} 97 \mathrm{X}-\mathrm{D}$. Overall, at the M06-2X level, the rate-determining barriers of the preferred stepwise mechanism for motors $\mathbf{2 a}-\mathbf{2} \mathbf{c}$ are 24, 44 and $13 \mathrm{~kJ} \mathrm{~mol}^{-1}$, respectively, which are similar to (or even smaller than) the corresponding $\omega \mathrm{B} 97 \mathrm{X}-\mathrm{D}$ estimates of 18,41 and $24 \mathrm{~kJ} \mathrm{~mol}^{-1}$. Given that M06-2X yields a rate-determining barrier of $39 \mathrm{~kJ} \mathrm{~mol}^{-1}$ for reference motor $\mathbf{1 c}{ }^{[29]}$ which is close to the $43 \mathrm{~kJ} \mathrm{~mol}^{-1}$ predicted by $\omega \mathrm{B} 97 \mathrm{X}-\mathrm{D},{ }^{[29]}$ our proposal that motors $2 \mathrm{a}$ and $2 \mathrm{c}$ achieve significant acceleration of the thermal isomerizations relative to reference motor $\mathbf{1 c}$ is also supported by M06-2X.

\section{Conclusions}

We have reported a computational study aimed at improving the thermal isomerization performance of overcrowded alkene-based rotary molecular motors through a structural redesign of some of the fastest available motors of this type,${ }^{[15,19]}$ wherein the sizes of the rotator and stator motor halves are substantially reduced. To this end, we have designed 
three new motors $\mathbf{2 a}-\mathbf{2 c}$, all featuring a synthetically viable indanylidene rotator ${ }^{[16,24]}$ and a six-membered diene ring stator, but differing in the stator bridging atom $\left(\mathrm{X}=\mathrm{CH}_{2}\right.$ or $\mathrm{O}$ or S).

First, by performing multiconfigurational quantum chemical calculations, it is demonstrated that the $E \rightarrow Z$ and $Z \rightarrow E$ photoisomerizations of motors $\mathbf{2 a - 2 c}$ occur in a unidirectional fashion and produce rotary motion. Then, by using DFT calculations to explore the mechanisms for the thermal isomerizations, one stepwise and one concerted mechanism are identified for these processes. The stepwise mechanism occurs in two steps via a syn-folded intermediate, where the first step involves a $P \rightarrow M$ rotator helicity inversion and the second step a syn $\rightarrow$ anti change in relative rotator-stator folding. The concerted mechanism, in turn, proceeds without any intermediate(s) but through a highly asynchronous TS in which no change in rotator helicity is yet manifested.

From the calculations, it is observed that the free-energy barriers of the two thermal mechanisms vary considerably between the motors. Further, it is found that this variation can be explained in terms of how a change in the size of the stator bridging atom contributes differently to steric repulsion in reactants and TSs. For motors $\mathbf{2 a}$ and $\mathbf{2 b}$, the stepwise mechanism is kinetically preferred with a rate-determining barrier of 18 and $41 \mathrm{~kJ} \mathrm{~mol}^{-1}$, respectively. For motor $\mathbf{2 c}$, the two mechanisms show similarly favorable kinetics with barriers of $23-24 \mathrm{~kJ} \mathrm{~mol}^{-1}$.

Additionally, it is also observed that the calculated rate-determining thermal isomerization barriers for the newly designed motors $\mathbf{2 a}-\mathbf{2} \mathbf{c}$ are up to $25 \mathrm{~kJ} \mathrm{~mol}^{-1}$ smaller than the corresponding barrier previously calculated for reference motor $1 \mathbf{1 c}^{\left[{ }^{[2]}\right.}$ Also, the motors isomerize through fewer steps than motor $\mathbf{1 c}^{[29]}$ (and other overcrowded alkene- 
based motors $)^{[21,41]}$ and without the negative impact of a competing side reaction. ${ }^{[2]}$ These mechanistic differences can be attributed to the lack of syn- or anti-folded conformations of the photoisomerization products of motors $\mathbf{2 a - 2}$. Taken together, the results of this work suggest a conformational approach to the design of overcrowded alkene-based motors with better thermal isomerization performance than existing motors of this type.

\section{Acknowledgements}

We acknowledge financial support from the Swedish Research Council (grant 621-20114353), the Olle Engkvist Foundation (grant 2014/734), the Carl Trygger Foundation (grant CTS 15:134) and Linköping University, as well as grants of computing time at the National Supercomputer Centre (NSC) in Linköping.

Keywords: density functional calculations $\bullet$ isomerization $\bullet$ molecular motors $\bullet$ rotary rates $\bullet$ stepwise or concerted 


\section{References}

[1] G. S. Kottas, L. I. Clarke, D. Horinek, J. Michl, Chem. Rev. 2005, 105, 12811376.

[2] J. Michl, E. C. H. Sykes, ACS Nano 2009, 3, 1042-1048.

[3] S. Erbas-Cakmak, D. A. Leigh, C. T. McTernan, A. L. Nussbaumer, Chem. Rev. 2015, 115, 10081-10206.

[4] C. Cheng, J. F. Stoddart, ChemPhysChem 2016, 17, 1780-1793.

[5] W. R. Browne, B. L. Feringa, Nat. Nanotechnol. 2006, 1, 25-35.

[6] E. R. Kay, D. A. Leigh, F. Zerbetto, Angew. Chem. Int. Ed. 2007, 46, 72-191.

[7] V. Balzani, A. Credi, M. Venturi, Chem. Soc. Rev. 2009, 38, 1542-1550.

[8] N. Harada, N. Koumura, B. L. Feringa, J. Am. Chem. Soc. 1997, 119, 7256-7264.

[9] N. Koumura, R. W. J. Zijlstra, R. A. van Delden, N. Harada, B. L. Feringa, Nature 1999, 401, 152-155.

[10] N. Koumura, E. M. Geertsema, M. B. van Gelder, A. Meetsma, B. L. Feringa, J. Am. Chem. Soc. 2002, 124, 5037-5051.

[11] M. K. J. ter Wiel, R. A. van Delden, A. Meetsma, B. L. Feringa, J. Am. Chem. Soc. 2003, 125, 15076-15086.

[12] D. Pijper, R. A. van Delden, A. Meetsma, B. L. Feringa, J. Am. Chem. Soc. 2005, 127, 17612-17613.

[13] J. Vicario, M. Walko, A. Meetsma, B. L. Feringa, J. Am. Chem. Soc. 2006, 128, 5127-5135.

[14] M. M. Pollard, M. Klok, D. Pijper, B. L. Feringa, Adv. Funct. Mater. 2007, 17, 718-729.

[15] M. Klok, N. Boyle, M. T. Pryce, A. Meetsma, W. R. Browne, B. L. Feringa, J. Am. Chem. Soc. 2008, 130, 10484-10485.

[16] M. M. Pollard, A. Meetsma, B. L. Feringa, Org. Biomol. Chem. 2008, 6, 507-512.

[17] M. M. Pollard, P. V. Wesenhagen, D. Pijper, B. L. Feringa, Org. Biomol. Chem. 2008, 6, 1605-1612.

[18] N. Ruangsupapichat, M. M. Pollard, S. R. Harutyunyan, B. L. Feringa, Nat. Chem. 2010, 3, 53-60.

[19] J. Vachon, G. T. Carroll, M. M. Pollard, E. M. Mes, A. M. Brouwer, B. L. Feringa, Photochem. Photobiol. Sci. 2014, 13, 241-246.

[20] J. Bauer, L. Hou, J. C. M. Kistemaker, B. L. Feringa, J. Org. Chem. 2014, 79, 4446-4455.

[21] A. Cnossen, J. C. M. Kistemaker, T. Kojima, B. L. Feringa, J. Org. Chem. 2014, 79, 927-935.

[22] J. Conyard, A. Cnossen, W. R. Browne, B. L. Feringa, S. R. Meech, J. Am. Chem. Soc. 2014, 136, 9692-9700.

[23] J. C. M. Kistemaker, P. Štacko, J. Visser, B. L. Feringa, Nat. Chem. 2015, 7, 890896.

[24] M. Guentner, M. Schildhauer, S. Thumser, P. Mayer, D. Stephenson, P. J. Mayer, H. Dube, Nat. Commun. 2015, 6, 8406. 
[25] L. Greb, J.-M. Lehn, J. Am. Chem. Soc. 2014, 136, 13114-13117.

[26] L. Greb, A. Eichöfer, J.-M. Lehn, Angew. Chem. Int. Ed. 2015, 54, 14345-14348.

[27] R. Augulis, M. Klok, B. L. Feringa, P. H. M. van Loosdrecht, Phys. Status Solidi C 2009, 6, 181-184.

[28] J. Conyard, K. Addison, I. A. Heisler, A. Cnossen, W. R. Browne, B. L. Feringa, S. R. Meech, Nat. Chem. 2012, 4, 547-551.

[29] B. Oruganti, C. Fang, B. Durbeej, Phys. Chem. Chem. Phys. 2015, 17, 2174021751.

[30] P.-T. Chiang, J. Mielke, J. Godoy, J. M. Guerrero, L. B. Alemany, C. J. Villagómez, A. Saywell, L. Grill, J. M. Tour, ACS Nano 2012, 6, 592-597.

[31] J. Chen, J. C. M. Kistemaker, J. Robertus, B. L. Feringa, J. Am. Chem. Soc. 2014, 136, 14924-14932.

[32] K. Gavvala, S. Satpathi, P. Hazra, RSC Adv. 2015, 5, 72793-72800.

[33] M. Klok, W. R. Browne, B. L. Feringa, Phys. Chem. Chem. Phys. 2009, 11, 91249131.

[34] S. Grimm, C. Bräuchle, I. Frank, ChemPhysChem 2005, 6, 1943-1947.

[35] A. Kazaryan, M. Filatov, J. Phys. Chem. A 2009, 113, 11630-11634.

[36] A. Kazaryan, J. C. M. Kistemaker, L. V Schäfer, W. R. Browne, B. L. Feringa, M. Filatov, J. Phys. Chem. A 2010, 114, 5058-5067.

[37] A. Kazaryan, Z. Lan, L. V. Schäfer, W. Thiel, M. Filatov, J. Chem. Theory Comput. 2011, 7, 2189-2199.

[38] F. Liu, K. Morokuma, J. Am. Chem. Soc. 2012, 134, 4864-4876.

[39] M. Filatov, M. Olivucci, J. Org. Chem. 2014, 79, 3587-3600.

[40] R. W. J. Zijlstra, W. F. Jager, B. de Lange, P. Th. van Duijnen, B. L. Feringa, H. Goto, A. Saito, N. Koumura, N. Harada, J. Org. Chem. 1999, 64, 1667-1674.

[41] G. Pérez-Hernández, L. González, Phys. Chem. Chem. Phys. 2010, 12, 1227912289.

[42] C. Fang, B. Oruganti, B. Durbeej, RSC. Adv. 2014, 4, 10240-10251.

[43] Y. Amatatsu, J. Phys. Chem. A 2011, 115, 13611-13618.

[44] Y. Amatatsu, J. Phys. Chem. A 2012, 116, 10182-10193.

[45] C. García-Iriepa, M. Marazzi, F. Zapata, A. Valentini, D. Sampedro, L. M. Frutos, J. Phys. Chem. Lett. 2013, 4, 1389-1396.

[46] G. Marchand, J. Eng, I. Schapiro, A. Valentini, L. M. Frutos, M. Olivucci, J. Léonard, E. Gindensperger, J. Phys. Chem. Lett. 2015, 6, 599-604.

[47] A. Nikiforov, J. A. Gamez, W. Thiel, M. Filatov, J. Phys. Chem. Lett. 2016, 7, $105-110$.

[48] J.-D. Chai, M. Head-Gordon, Phys. Chem. Chem. Phys. 2008, 10, 6615-6620.

[49] A. V Marenich, C. J. Cramer, D. G. Truhlar, J. Phys. Chem. B 2009, 113, 63786396.

[50] S. Schenker, C. Schneider, S. B. Tsogoeva, T. Clark, J Chem. Theory Comput. 2011, 7, 3586-3595.

[51] S. Grimme, J. Comput. Chem. 2004, 25, 1463-1473.

[52] J. Antony, S. Grimme, Phys. Chem. Chem. Phys. 2006, 8, 5287-5293. 
[53] B. O. Roos, P. R. Taylor, P. E. M. Siegbahn, Chem. Phys. 1980, 48, 157-173.

[54] K. Andersson, P.-Å. Malmqvist, B. O. Roos, A. J. Sadlej, K. Wolinski, J. Phys. Chem. 1990, 94, 5483-5488.

[55] K. Andersson, P.-Å. Malmqvist, B. O. Roos, J. Chem. Phys. 1992, 96, 1218-1226.

[56] H. P. Hratchian, H. B. Schlegel, J. Chem. Phys. 2004, 120, 9918-9924.

[57] Gaussian 09 (Revision D.01), M. J. Frisch, G. W. Trucks, H. B. Schlegel, G. E. Scuseria, M. A. Robb, J. R. Cheeseman, G. Scalmani, V. Barone, B. Mennucci, G. A. Petersson, H. Nakatsuji, M. Caricato, X. Li, H. P. Hratchian, A. F. Izmaylov, J. Bloino, G. Zheng, J. L. Sonnenberg, M. Hada, M. Ehara, K. Toyota, R. Fukuda, J. Hasegawa, M. Ishida, T. Nakajima, Y. Honda, O. Kitao, H. Nakai, T. Vreven, J. A. Montgomery, Jr., J. E. Peralta, F. Ogliaro, M. Bearpark, J. J. Heyd, E. Brothers, K. N. Kudin, V. N. Staroverov, R. Kobayashi, J. Normand, K. Raghavachari, A. Rendell, J. C. Burant, S. S. Iyengar, J. Tomasi, M. Cossi, N. Rega, J. M. Millam, M. Klene, J. E. Knox, J. B. Cross, V. Bakken, C. Adamo, J. Jaramillo, R. Gomperts, R. E. Stratmann, O. Yazyev, A. J. Austin, R. Cammi, C. Pomelli, J. W. Ochterski, R. L. Martin, K. Morokuma, V. G. Zakrzewski, G. A. Voth, P. Salvador, J. J. Dannenberg, S. Dapprich, A. D. Daniels, Ö. Farkas, J. B. Foresman, J. V. Ortiz, J. Cioslowski, D. J. Fox, Gaussian, Inc., Wallingford CT, USA, 2009.

[58] F. Aquilante, J. Autschbach, R. K. Carlson, L. F. Chibotaru, M. G. Delcey, L. De Vico, I. Fdez. Galván, N. Ferré, L. M. Frutos, L. Gagliardi, M. Garavelli, A. Giussani, C. E. Hoyer, G. Li Manni, H. Lischka, D. Ma, P.-Å. Malmqvist, T. Müller, A. Nenov, M. Olivucci, T. B. Pedersen, D. Peng, F. Plasser, B. Pritchard, M. Reiher, I. Rivalta, I. Schapiro, J. Segarra-Martí, M. Stenrup, D. G. Truhlar, L. Ungur, A. Valentini, S. Vancoillie, V. Veryazov, V. P. Vysotskiy, O. Weingart, F. Zapata, R. Lindh, J. Comput. Chem. 2016, 37, 506-541.

[59] M. Klok, M. Walko, E. M. Geertsema, N. Ruangsupapichat, J. C. M. Kistemaker, A. Meetsma, B. L. Feringa, Chem. Eur. J. 2008, 14, 11183-11193.

[60] B. A. Hess Jr., L. Smentak, Org. Biomol. Chem. 2012, 10, 7503-7509.

[61] M. G. Memeo, P. Quadrelli, Chem. Eur. J. 2012, 18, 12554-12582.

[62] E. H. Krenske, S. He, J. Huang, Y. Du, K. N. Houk, R. P. Hsung, J. Am. Chem. Soc. 2013, 135, 5242-5245.

[63] Y. Zhao, D. G. Truhlar, Theor. Chem. Acc. 2008, 120, 215-241. 


\section{Table of Contents}

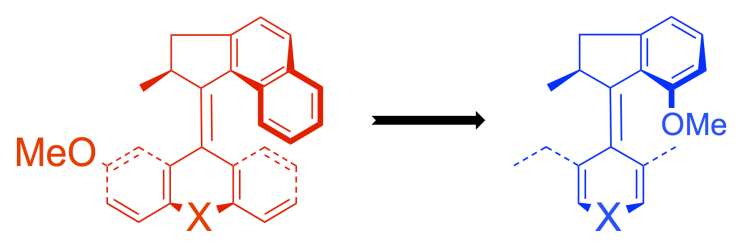

Smaller but faster: Reducing the sizes of the rotator and stator halves (see picture) of some of the fastest available overcrowded alkene-based molecular motors is found to lower the thermal isomerization barriers by up to $25 \mathrm{~kJ} \mathrm{~mol}^{-1}$ and facilitate the isomerization process by reducing the number of steps involved. 


\section{Computational Insight to Improve the Thermal Isomerization Performance of Overcrowded Alkene-Based Molecular Motors through Structural Redesign}

Baswanth Oruganti, Jun Wang, and Bo Durbeej*[a]

Supporting Information

[a] B. Oruganti, Dr. J. Wang, Prof. B. Durbeej

Division of Theoretical Chemistry, IFM

Linköping University

58183 Linköping (Sweden)

E-mail:bodur@ifm.liu.se 


\section{Contents}

Supplementary Computational Details

Figure S1

Figure S2

Table S1

Table S2

Table S3

Table S4

Table S5

Table S6

References for this document

Cartesian coordinates for stationary points of motors $\mathbf{2 a - 2 c}$ pages $\mathrm{S} 3$

pages $\mathrm{S} 4-\mathrm{S} 9$

page $\mathrm{S} 10$

page $\mathrm{S} 11$

page S12

page $\mathrm{S} 13$

page S14

page S15

page S16

page S17

pages S18-S56 


\section{Supplementary Computational Details}

To verify that the $E \rightarrow Z$ and $Z \rightarrow E$ photoisomerizations of motors $2 \mathbf{2 a}-\mathbf{2 c}$ produce unidirectional rotary motion, the geometric relaxation of the light-absorbing anti-(M)stable- $E$ and anti-( $(M)$-stable- $Z$ isomers from the vertically excited Franck-Condon (FC) points in the lowest excited singlet state $\left(\mathrm{S}_{1}\right)$ populated by light absorption was modeled using $\mathrm{CASSCF}^{[1]}$ and CASPT2 ${ }^{[2-4]}$ in combination with the SVP basis set. Throughout, active spaces comprising either $12 \pi$-electrons in 12 orbitals (for motor $\mathbf{2 a}$, see Figure S1 below), or 14 electrons ( $12 \pi+$ one lone pair of the stator heteroatom) in 13 orbitals (for motors $\mathbf{2 b}$ and $\mathbf{2 c}$ ), were employed. The procedure was as follows.

First, the ground-state $\left(\mathrm{S}_{0}\right)$ geometries of anti- $(M)$-stable- $E$ and anti- $(M)$-stable- $Z$ were optimized with CASSCF. Single point calculations were then performed to obtain the $\mathrm{S}_{0}$ and $\mathrm{S}_{1}$ energies at the resulting geometries. For the ground state, this was achieved by performing (single-state) CASPT2 calculations. For the excited state, this was achieved by first performing state-averaged CASSCF (SA-CASSCF) calculations with equal (0.5) weights for $\mathrm{S}_{0}$ and $\mathrm{S}_{1}$, followed by multi-state CASPT2 (MS-CASPT2) ${ }^{[4]}$ calculations. Second, the FC relaxation processes were modeled by optimizing the $S_{1}$ geometries of anti-(M)-stable- $E$ and anti-( $M)$-stable- $Z$ using SA-CASSCF, again with equal weights for $S_{0}$ and $S_{1}$. Finally, the $S_{1}$ energies of the resulting minima, denoted anti-(M)-stable- $E^{*}$ and anti-( $\left.M\right)$-stable- $Z^{*}$, were obtained from MS-CASPT2 single point calculations. 


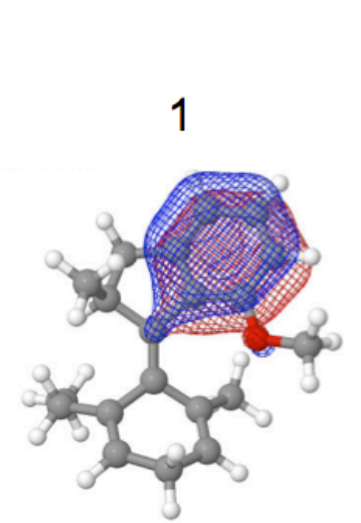

1.96

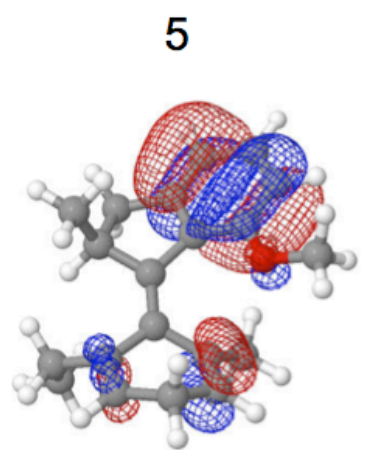

1.91

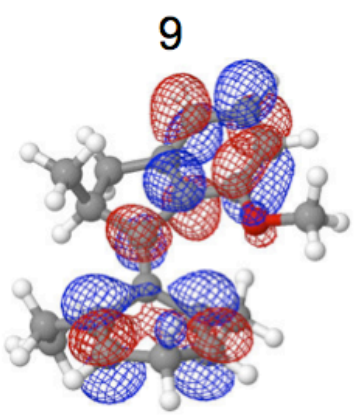

0.09

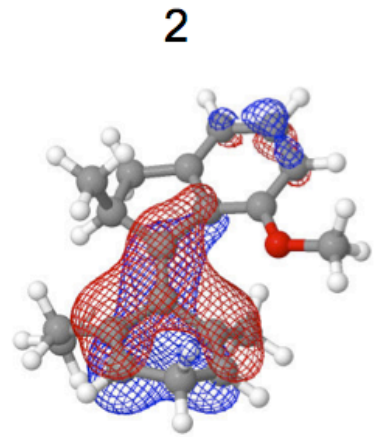

1.93

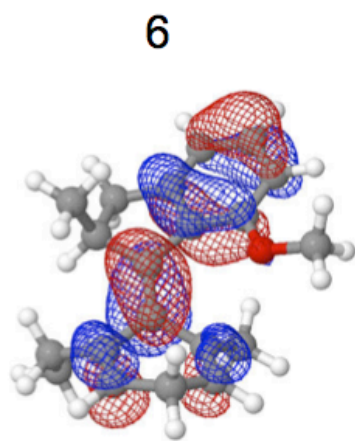

1.88

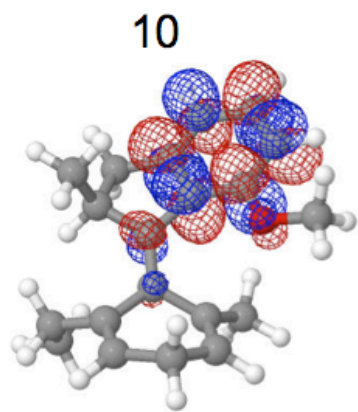

0.04
3

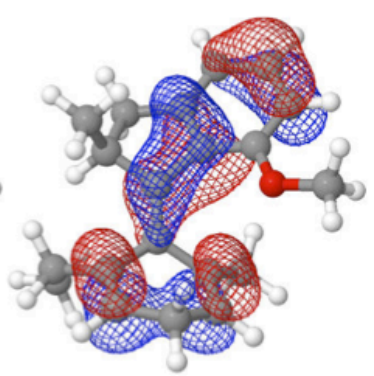

1.91

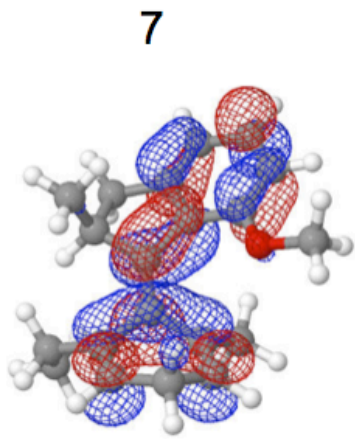

0.13

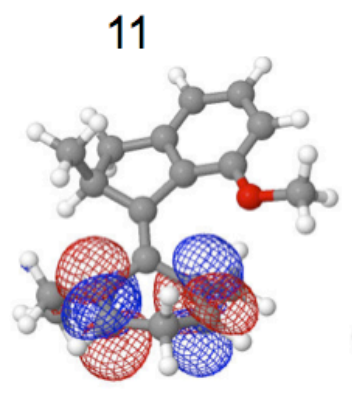

0.09

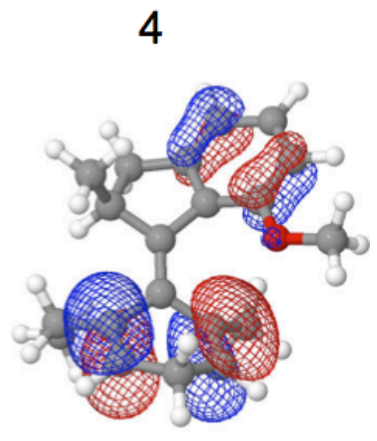

1.91

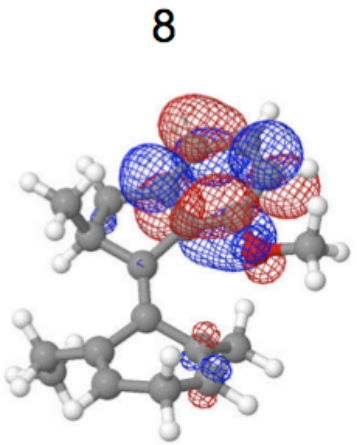

0.09

12

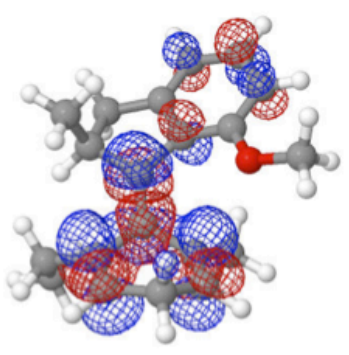

0.07

Figure S1. Active molecular orbitals and their occupation numbers in different CASSCFoptimized species of motor $\mathbf{2 a}$. 


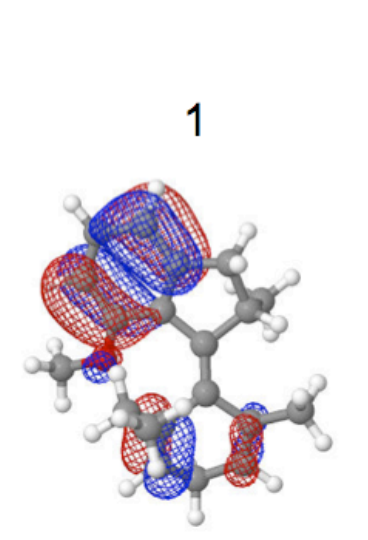

1.91

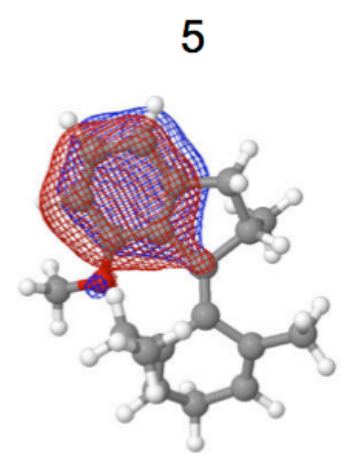

1.96

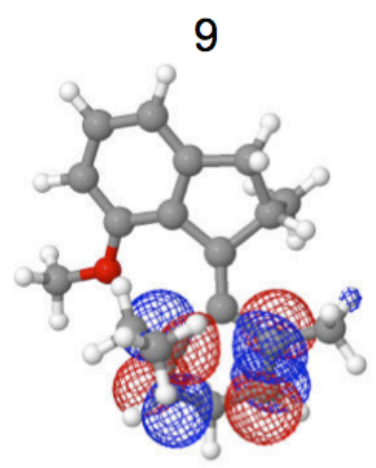

0.09
2

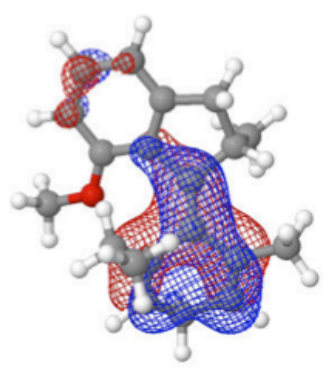

1.93

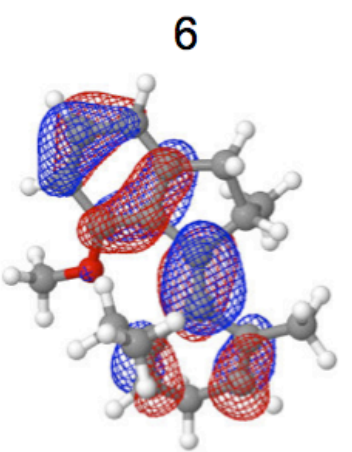

1.88

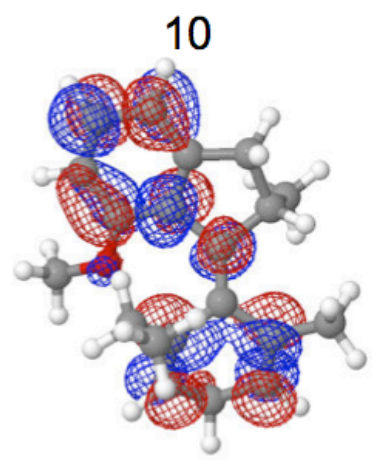

0.09
3

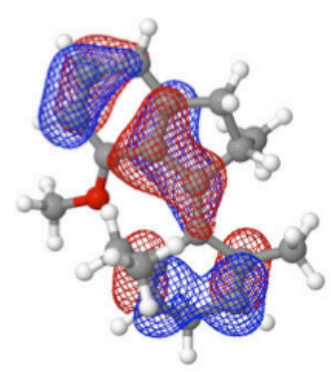

1.91

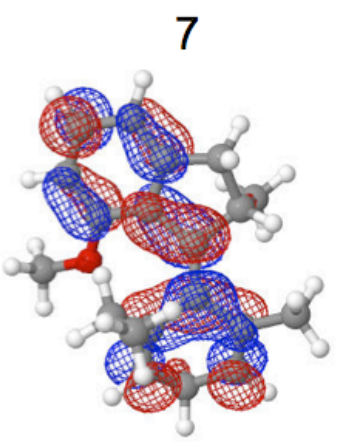

0.13

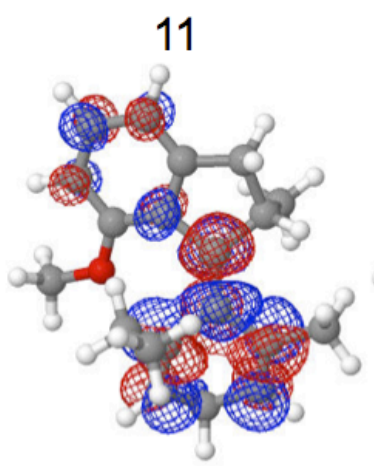

0.07
4

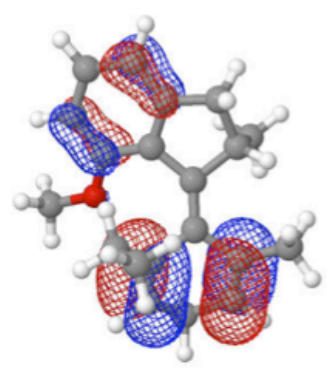

1.91
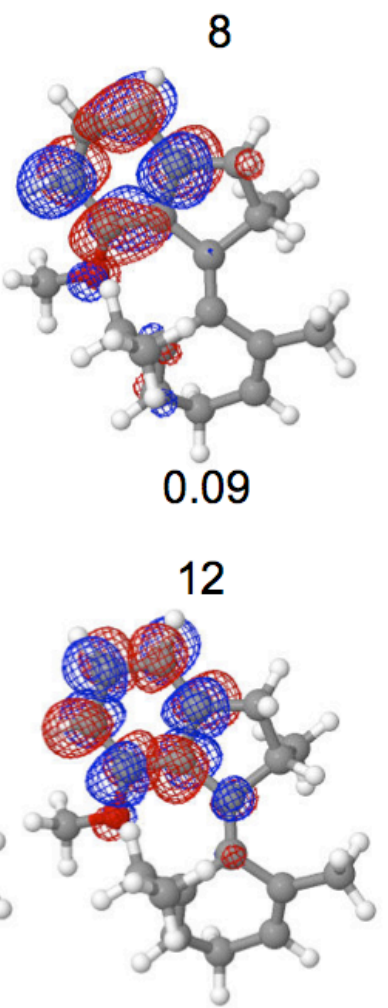

0.04

Figure S1. Continued 

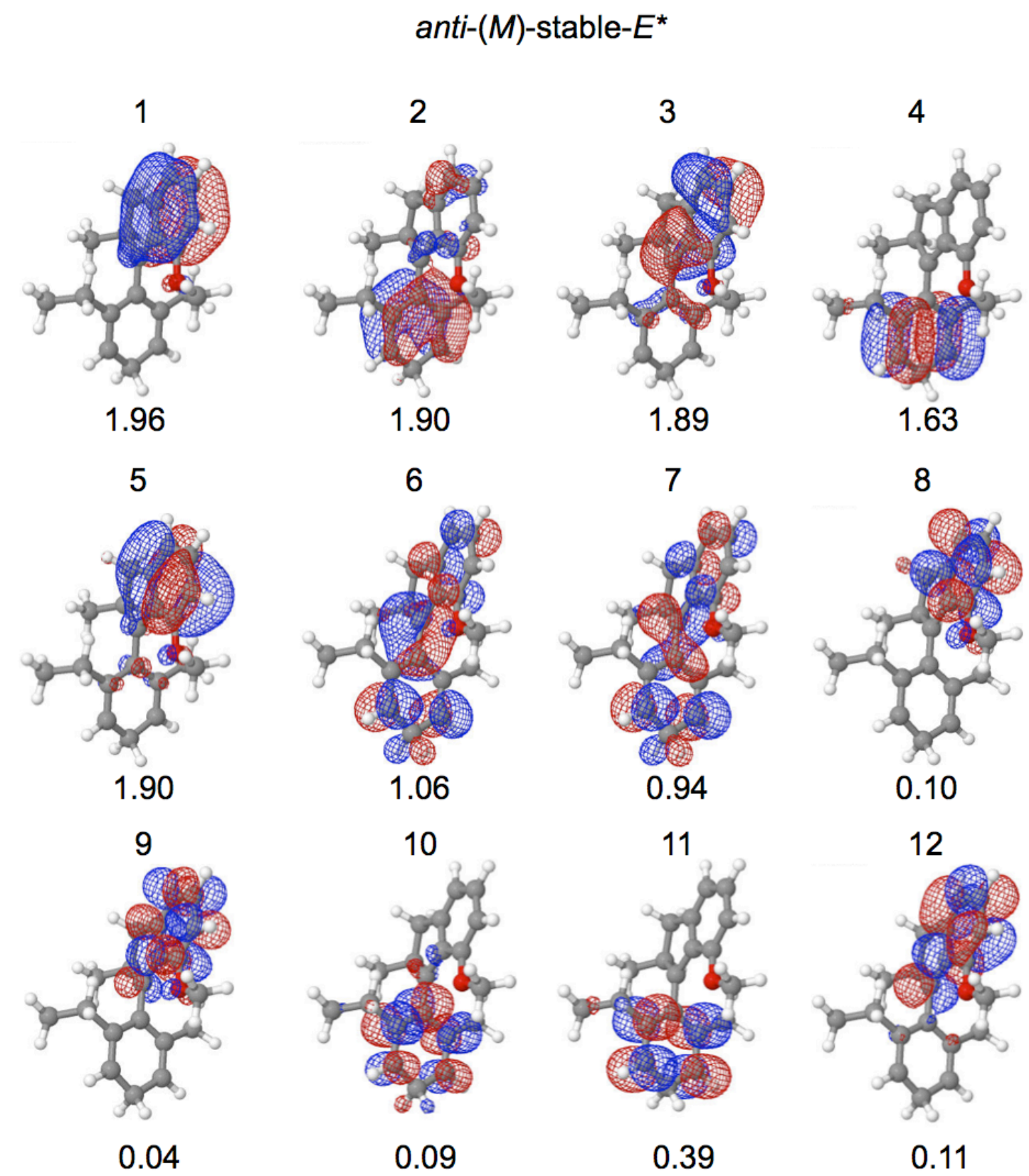

Figure S1. Continued
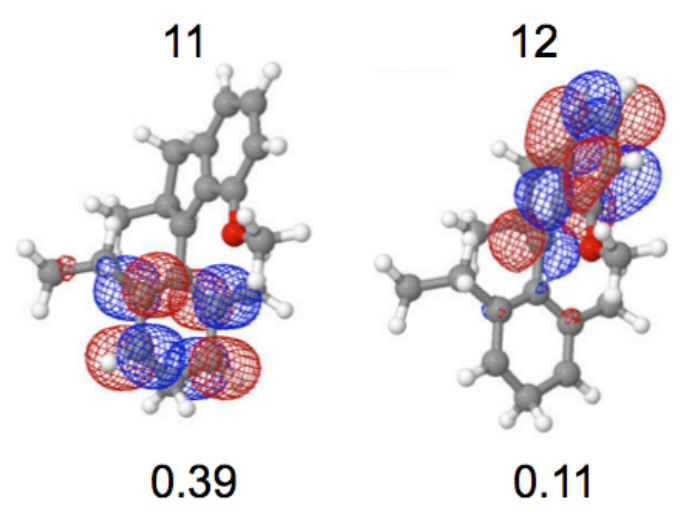

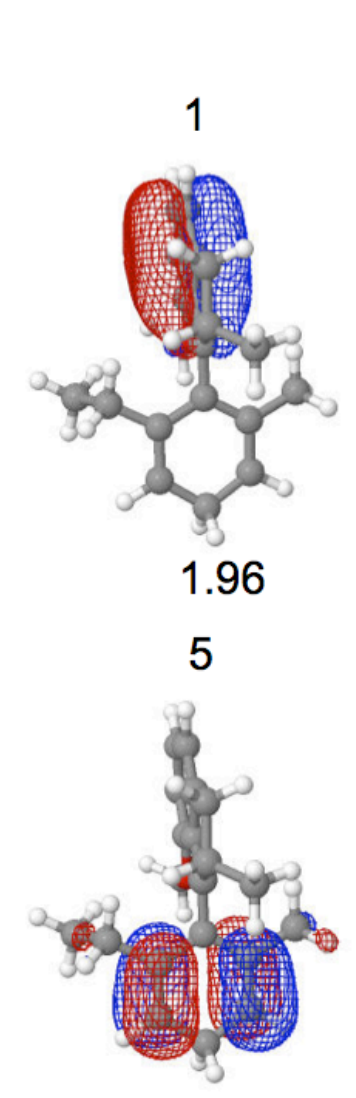

1.63

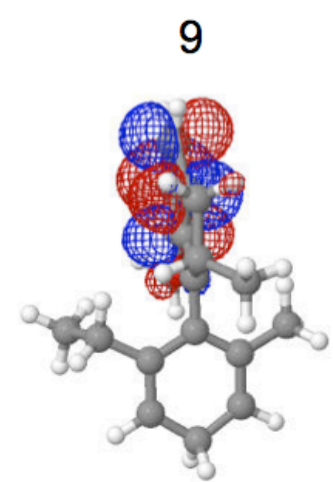

0.10
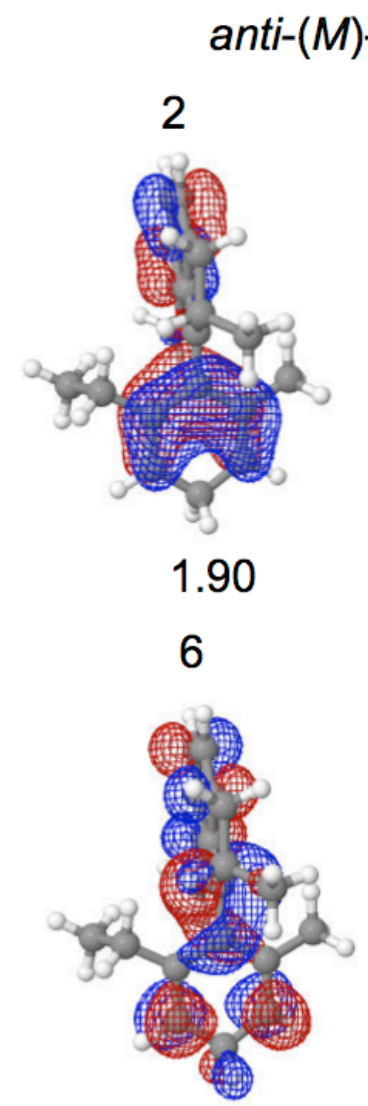

1.05

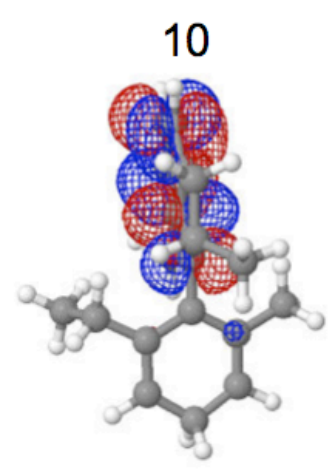

0.11

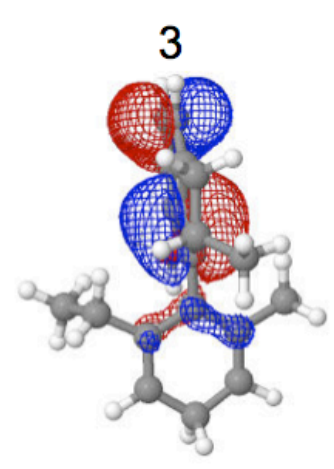

1.89

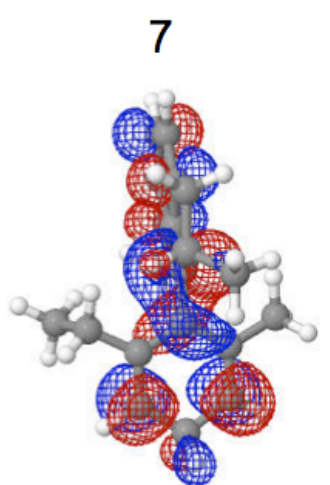

0.95

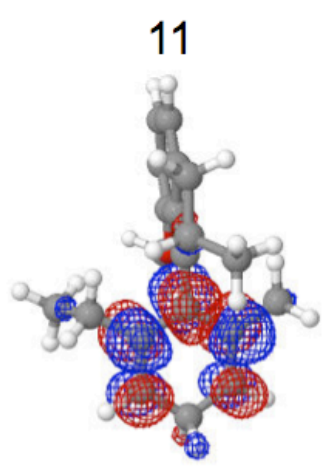

0.09

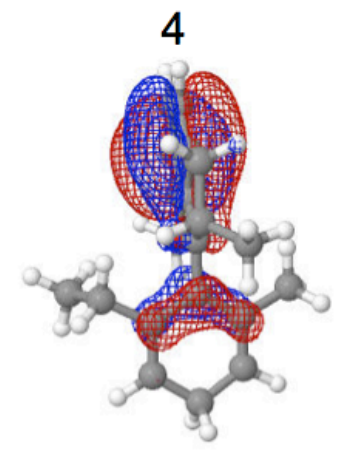

1.90

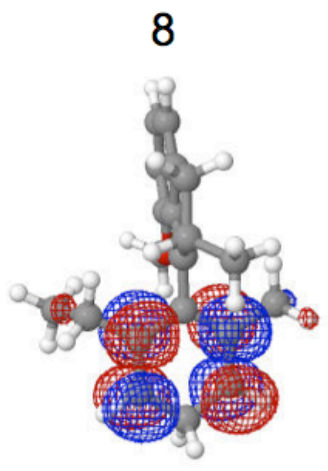

0.39

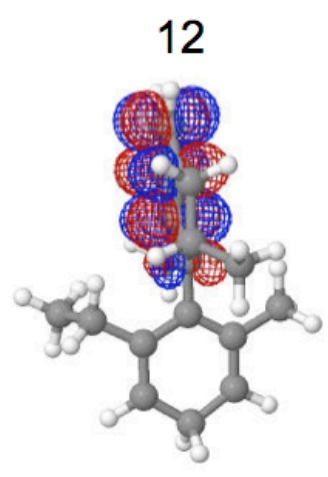

0.04

Figure S1. Continued 
$(P)$-unstable-Z

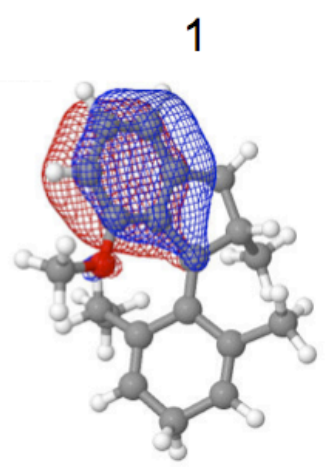

1.96

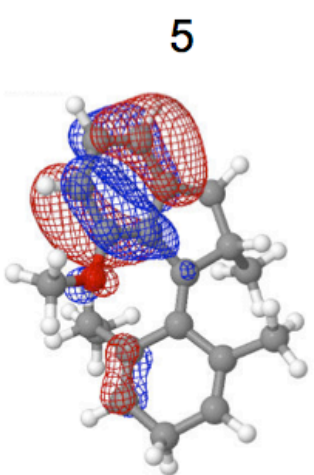

1.90

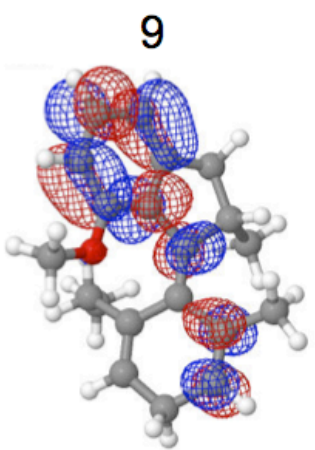

0.09

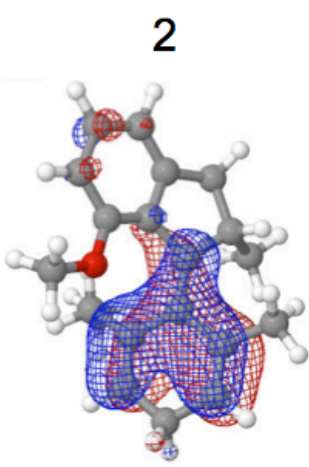

1.94

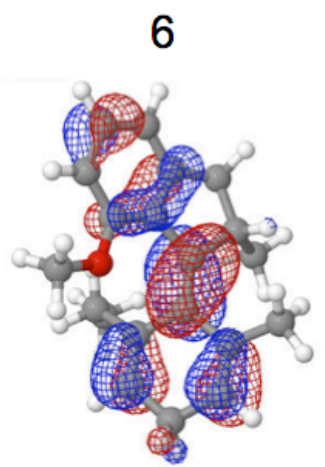

1.83

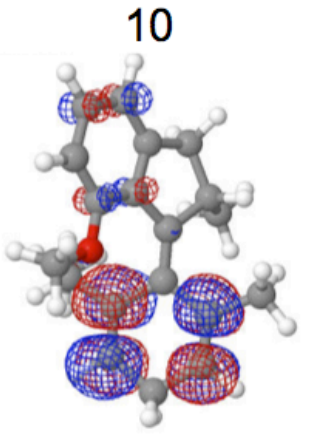

0.09

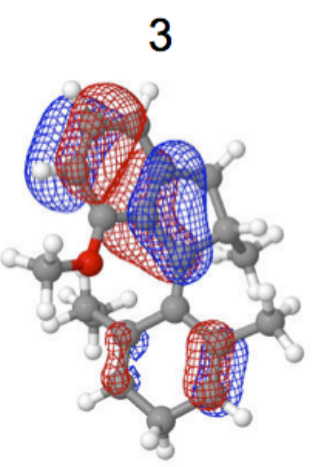

1.91

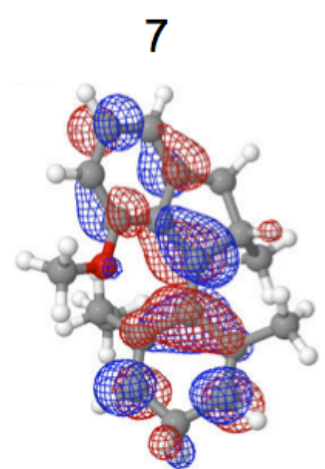

0.17

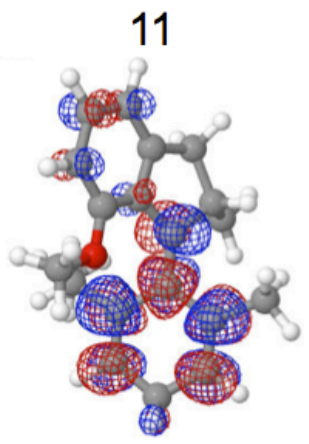

0.06

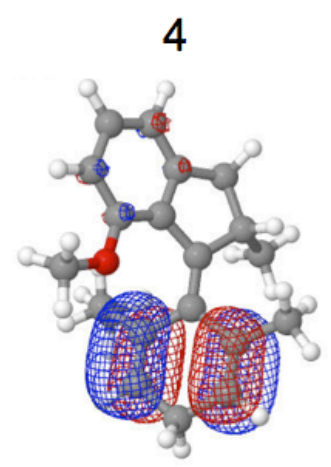

1.91

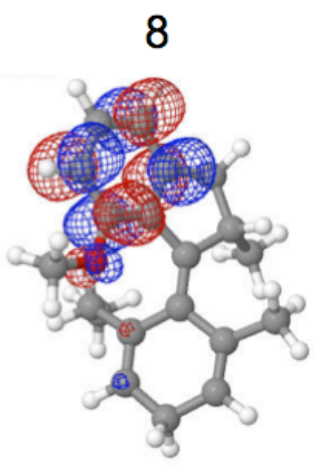

0.09

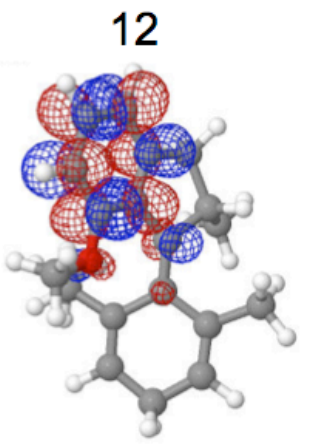

0.04

Figure S1. Continued 

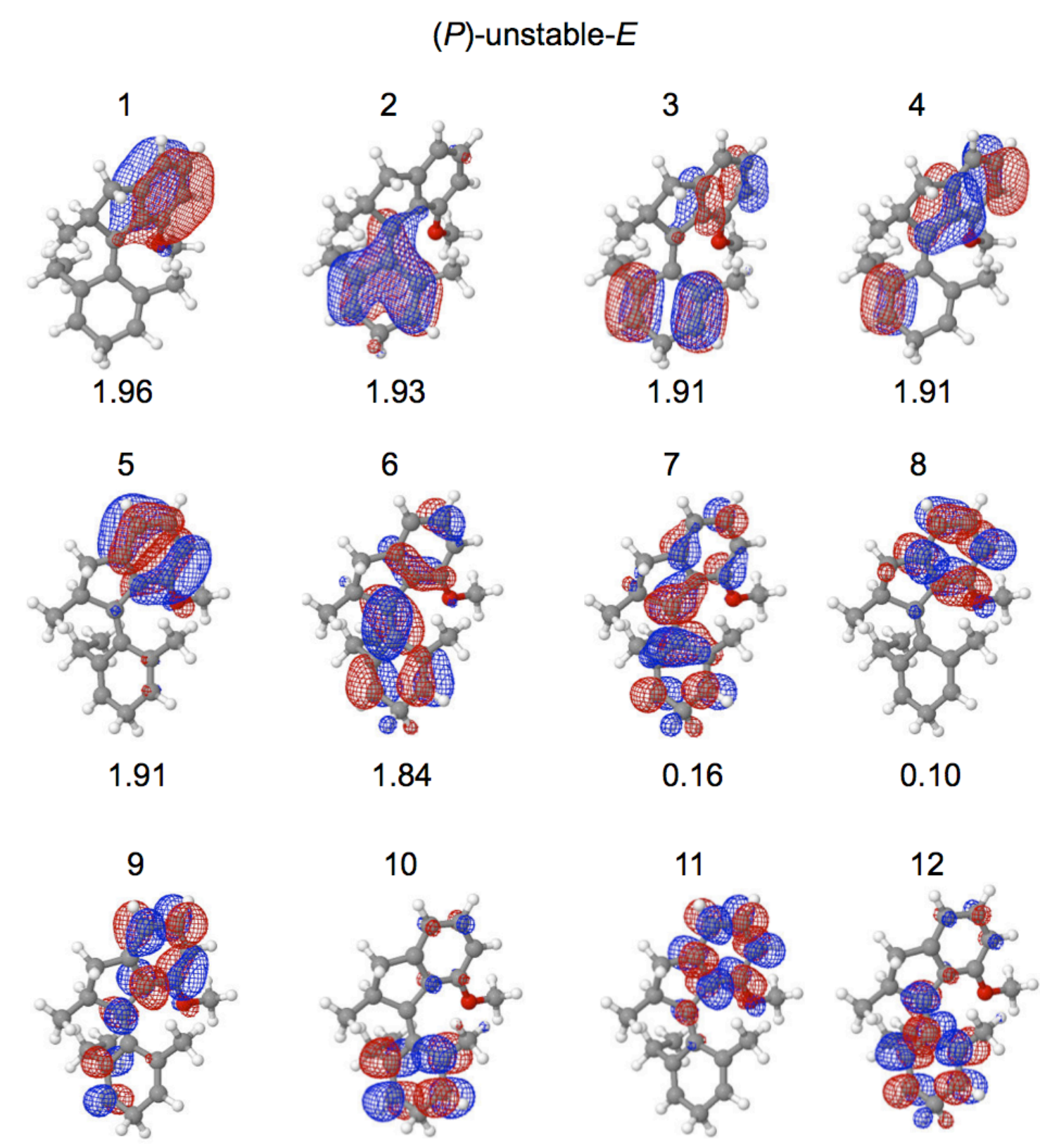

1.84

0.16

0.10

0.09
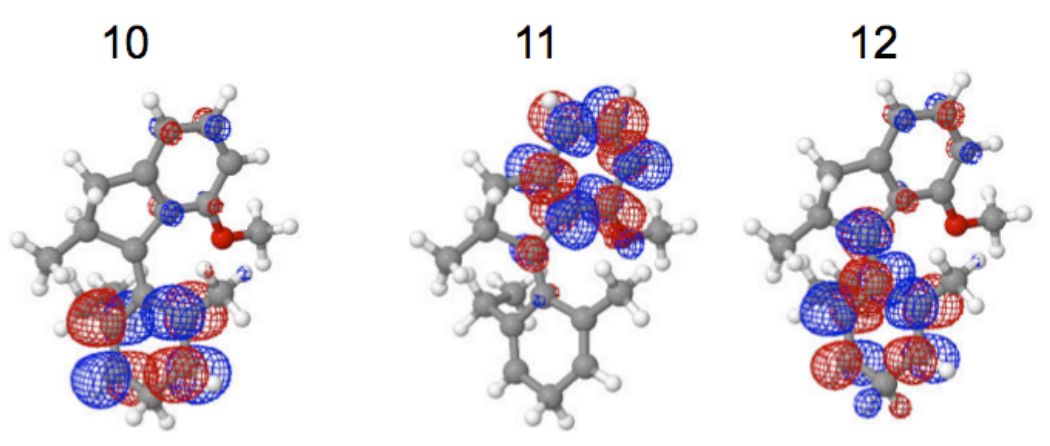

0.09

0.04

0.06

Figure S1. Continued 
$(P)$-unstable- $Z \rightarrow$ anti- $(M)$-stable- $Z \quad(P)$-unstable- $E \rightarrow$ anti- $(M)$-stable- $E$
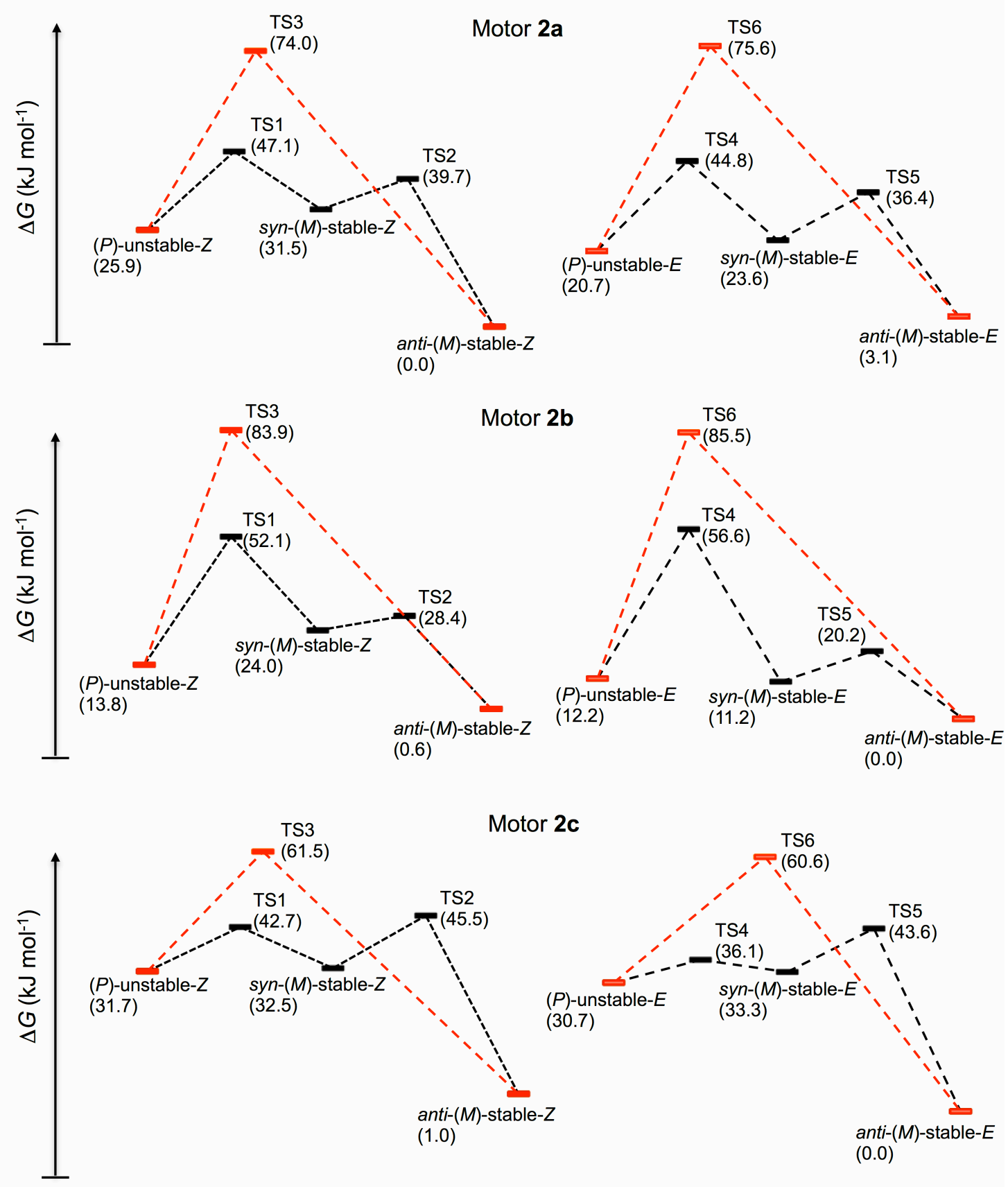

Figure S2. M06-2X free-energy profiles for stepwise and concerted mechanisms for the thermal isomerizations of motors $\mathbf{2 a}-\mathbf{2 c}$. 
Table S1. Relative CASPT2//CASSCF energies (in $\mathrm{kJ} \mathrm{mol}^{-1}$ ) of the lightabsorbing and photoproduct isomers of motors $2 \mathbf{a}-\mathbf{2} \mathbf{c}^{[\mathrm{a}]}$

$\begin{array}{lllll}\text { Motor } & \text { anti- }(M) \text {-stable- } E & (P) \text {-unstable- } E & \text { anti- }(M) \text {-stable- } Z & (P) \text {-unstable- } Z\end{array}$

$\begin{array}{lllll}2 \mathbf{a} & 1.2 & 24.3 & 0.0 & 27.0\end{array}$

2b $\quad 0.0$

11.7

2.2

16.0

2c 0.0

36.1

9.6

38.0

[a] All calculations carried out using the SVP basis set. 
Table S2. CASSCF geometric parameters (in $\AA$ and degrees) of the lightabsorbing isomers and the corresponding excited-state minima of motors $\mathbf{2 a}-\mathbf{2} \mathbf{c}^{[\mathrm{a}]}$

Motor

Stationary point

C4-C1'

$\alpha$

2a

anti-( $M)$-stable- $E$

1.365

167.6

anti- $(M)$-stable- $E^{*}$

1.496

89.1

anti-(M)-stable- $Z$

1.366

$-6.1$

anti- $(M)$-stable- $Z^{*}$

1.496

$-92.7$

2b

anti- $(M)$-stable- $E$

1.367

165.9

anti- $(M)$-stable- $E^{*}$

1.495

89.6

anti-(M)-stable-Z

1.367

$-9.2$

anti- $(M)$-stable- $Z^{*}$

1.494

$-93.5$

2c

anti-(M)-stable- $E$

1.362

172.6

anti- $(M)$-stable- $E^{*}$

1.499

89.4

anti-(M)-stable-Z

1.362

$-2.2$

anti- $(M)$-stable- $Z^{*}$

1.499

$-93.2$

[a] All calculations carried out using the SVP basis set. 
Table S3. Geometric parameters (in $\AA$ and degrees) of stationary points relevant for the thermal isomerizations of motors $2 \mathbf{a}^{[\mathrm{a}]}$

\begin{tabular}{lcccccc} 
Stationary point & C4-C1' & $\alpha$ & $\beta$ & $\gamma$ & $\delta$ & $\delta^{\prime}$ \\
\hline$(P)$-unstable- $Z$ & 1.377 & 36.5 & 25.1 & 40.2 & 9.1 & 16.1 \\
syn- $(M)$-stable- $Z$ & 1.361 & -9.1 & -60.6 & 119.0 & -51.9 & 51.0 \\
anti- $(M)$-stable- $Z$ & 1.362 & -10.7 & -29.3 & 90.6 & 38.4 & -46.0 \\
TS1 & 1.357 & 0.8 & -47.3 & 112.0 & -69.3 & 80.9 \\
TS2 & 1.372 & -20.7 & -52.6 & 115.7 & -25.4 & 10.5 \\
TS3 & 1.359 & 9.0 & 28.9 & 24.0 & 75.1 & -88.6 \\
$(P)$-unstable- $E$ & 1.376 & -144.0 & 29.3 & 34.8 & 6.0 & 17.7 \\
syn- $(M)$-stable- $E$ & 1.358 & -176.6 & -59.5 & 119.2 & 52.1 & -58.0 \\
anti- $(M)$-stable- $E$ & 1.362 & 166.0 & -28.8 & 90.1 & -46.9 & 43.1 \\
TS4 & 1.357 & 2.2 & -42.2 & 109.4 & 79.0 & -70.4 \\
TS5 & 1.365 & -177.4 & -61.4 & 122.4 & 36.1 & -44.7 \\
TS6 & 1.360 & 172.9 & 26.5 & 27.3 & -87.7 & 74.2 \\
\hline
\end{tabular}

[a] All calculations performed at the $\omega \mathrm{B} 97 \mathrm{X}-\mathrm{D} / \mathrm{SVP}$ level of theory using an SMD description of the dichloromethane solvent. 
Table S4. Geometric parameters (in $\AA$ and degrees) of stationary points relevant for the thermal isomerizations of motor $\mathbf{2} \mathbf{b} .^{[a]}$

\begin{tabular}{lcccccc} 
Stationary point & C4-C1' & $\alpha$ & $\beta$ & $\gamma$ & $\delta$ & $\delta^{\prime}$ \\
\hline$(P)$-unstable- $Z$ & 1.378 & 34.3 & 25.3 & 41.6 & 8.2 & 16.5 \\
syn- $(M)$-stable- $Z$ & 1.368 & -12.7 & -57.2 & 116.6 & -34.4 & 28.2 \\
anti- $(M)$-stable- $Z$ & 1.365 & -14.0 & -34.8 & 97.7 & 23.9 & -36.0 \\
TS1 & 1.359 & 2.3 & -37.4 & 104.0 & -64.1 & 73.7 \\
TS2 & 1.369 & -14.4 & -56.0 & 116.0 & -30.9 & 22.4 \\
TS3 & 1.364 & 15.2 & 19.6 & 37.2 & 65.5 & -76.6 \\
$(P)$-unstable- $E$ & 1.378 & -145.4 & 27.5 & 37.7 & 8.1 & 16.3 \\
syn- $(M)$-stable- $E$ & 1.373 & 161.8 & -44.6 & 109.7 & -10.0 & -12.6 \\
anti- $(M)$-stable- $E$ & 1.364 & 165.1 & -31.1 & 92.7 & -39.5 & 32.3 \\
TS4 & 1.358 & -158.1 & -30.7 & 99.6 & 76.1 & -66.3 \\
TS5 & 1.369 & 159.5 & -31.7 & 92.4 & -33.7 & 21.7 \\
TS6 & 1.365 & 179.3 & 15.4 & 40.9 & -74.4 & 65.0 \\
\hline
\end{tabular}

[a] All calculations performed at the $\omega \mathrm{B} 97 \mathrm{X}-\mathrm{D} / \mathrm{SVP}$ level of theory using an SMD description of the dichloromethane solvent. 
Table S5. Geometric parameters (in $\AA$ and degrees) of stationary points relevant for the thermal isomerizations of motor $\mathbf{2 c} .^{[a]}$

\begin{tabular}{lcccccc|} 
Stationary point & C4-C1' & $\alpha$ & $\beta$ & $\gamma$ & $\delta$ & $\delta^{\prime}$ \\
\hline$(P)$-unstable- $Z$ & 1.384 & 36.5 & 22.3 & 42.3 & 6.1 & 20.1 \\
syn- $(M)$-stable- $Z$ & 1.356 & -5.2 & -58.7 & 119.1 & -64.4 & 65.0 \\
anti- $(M)$-stable- $Z$ & 1.361 & -9.1 & -27.2 & 88.3 & 44.9 & -50.8 \\
TS1 & 1.355 & -2.6 & -53.5 & 115.7 & -67.1 & 69.5 \\
TS2 & 1.377 & -20.2 & -54.2 & 117.6 & -22.9 & 6.8 \\
TS3 & 1.356 & 10.8 & 42.4 & 12.2 & 74.7 & -83.8 \\
$(P)$-unstable- $E$ & 1.382 & -143 & 29.5 & 32.8 & 5.7 & 19.6 \\
syn- $(M)$-stable- $E$ & 1.358 & -176.6 & -59.5 & 119.2 & 52.1 & -58.0 \\
anti- $(M)$-stable- $E$ & 1.360 & 170.6 & -26.5 & 87.1 & -51.3 & 49.2 \\
TS4 & 1.374 & -157.1 & 9.9 & 72.3 & 45.4 & -33.7 \\
TS5 & 1.375 & 165.2 & -55.8 & 118.5 & 11.4 & -27.2 \\
TS6 & 1.355 & 172.6 & 38.1 & 15.1 & -87.2 & 76.9 \\
\hline
\end{tabular}

[a] All calculations performed at the $\omega \mathrm{B} 97 \mathrm{X}-\mathrm{D} / \mathrm{SVP}$ level of theory using an SMD description of the dichloromethane solvent. 
Table S6. Possible pair-wise fjord-region steric interactions between the rotator and stator in different stationary points relevant for the thermal isomerizations of motor $2 \mathbf{c}^{[\mathrm{a}]}$

Stationary poin

Steric interactions

Total $^{[\mathrm{b}]}$

$(P)$-unstable- $Z$

$\mathrm{C} 9^{\prime}-\mathrm{C} 9, \mathrm{C} 9^{\prime}-\mathrm{C} 4, \mathrm{C} 2^{\prime}-\mathrm{C} 9, \mathrm{C} 2^{\prime}-\mathrm{C} 5, \mathrm{O}^{\prime}-\mathrm{C} 7, \mathrm{O}^{\prime}-\mathrm{C} 3, \mathrm{C} 1^{\prime}-\mathrm{C} 7, \mathrm{C} 7{ }^{\prime}-\mathrm{C} 7, \mathrm{C} 7{ }^{\prime} \mathrm{a}-\mathrm{C} 3$

9

syn-(M)-stable- $Z$

$\mathrm{C} 2^{\prime}-\mathrm{C} 9, \mathrm{C} 2^{\prime}-\mathrm{C} 5, \mathrm{O}^{\prime}-\mathrm{C} 7, \mathrm{O}^{\prime}-\mathrm{C} 8, \mathrm{C} 7{ }^{\prime} \mathrm{a}-\mathrm{C} 3$

TS1

$\mathrm{C} 2{ }^{\prime}-\mathrm{C} 9, \mathrm{C} 2^{\prime}-\mathrm{C} 5, \mathrm{O} 8^{\prime}-\mathrm{C} 7, \mathrm{C} 7{ }^{\prime} \mathrm{a}-\mathrm{C} 3$

5

TS2

$\mathrm{C} 9{ }^{\prime}-\mathrm{C} 9, \mathrm{C} 2^{\prime}-\mathrm{C} 9, \mathrm{C} 2^{\prime}-\mathrm{C} 5, \mathrm{O}^{\prime}-\mathrm{C} 7, \mathrm{O}^{\prime}-\mathrm{C} 3, \mathrm{C} 1^{\prime}-\mathrm{C} 7, \mathrm{C} 7{ }^{\prime} \mathrm{a}-\mathrm{C} 3, \mathrm{C} 7{ }^{\prime}-\mathrm{C} 7$

4

TS3

$\mathrm{C} 9{ }^{\prime}-\mathrm{C} 9, \mathrm{C} 9^{\prime}-\mathrm{C} 5, \mathrm{C} 9^{\prime}-\mathrm{C} 4, \mathrm{C} 2^{\prime}-\mathrm{C} 5, \mathrm{O}^{\prime}-\mathrm{C} 7, \mathrm{C} 7{ }^{\prime} \mathrm{a}-\mathrm{C} 3$

8

(P)-unstable- $E$

$\mathrm{C} 9{ }^{\prime}-\mathrm{C} 3, \mathrm{C} 9^{\prime}-\mathrm{C} 7, \mathrm{C} 9{ }^{\prime}-\mathrm{C} 4, \mathrm{C} 2^{\prime}-\mathrm{C} 7, \mathrm{C} 2^{\prime}-\mathrm{C} 3, \mathrm{O}^{\prime}-\mathrm{C} 9, \mathrm{C} 7{ }^{\prime}-\mathrm{C} 9, \mathrm{C} 1^{\prime}-\mathrm{C} 7, \mathrm{C} 7{ }^{\prime} \mathrm{a}-\mathrm{C} 5, \mathrm{C} 1^{\prime}-\mathrm{C} 9$

6

syn- $(M)$-stable- $E$

$\mathrm{C} 2{ }^{\prime}-\mathrm{C} 7, \mathrm{C} 2{ }^{\prime}-\mathrm{C} 3, \mathrm{O}^{\prime}-\mathrm{C} 9, \mathrm{C} 7{ }^{\prime}-\mathrm{C} 9, \mathrm{C} 1^{\prime}-\mathrm{C} 7, \mathrm{C} 7{ }^{\prime} \mathrm{a}-\mathrm{C} 5, \mathrm{C} 1^{\prime}-\mathrm{C} 9$

10

TS4

$\mathrm{C} 2{ }^{\prime}-\mathrm{C} 7, \mathrm{C} 2^{\prime}-\mathrm{C} 3, \mathrm{O}^{\prime}-\mathrm{C} 9, \mathrm{C} 7{ }^{\prime} \mathrm{a}-\mathrm{C} 5, \mathrm{C} 1^{\prime}-\mathrm{C} 9$

7

TS5

$\mathrm{C} 9{ }^{\prime}-\mathrm{C} 7, \mathrm{C} 2^{\prime}-\mathrm{C} 7, \mathrm{C} 2^{\prime}-\mathrm{C} 3, \mathrm{O} 8^{\prime}-\mathrm{C} 9, \mathrm{C} 7{ }^{\prime}-\mathrm{C} 9, \mathrm{C} 1^{\prime}-\mathrm{C} 7, \mathrm{C} 7{ }^{\prime} \mathrm{a}-\mathrm{C} 5, \mathrm{C} 1^{\prime}-\mathrm{C} 9$

5

$\mathrm{C} 9{ }^{\prime}-\mathrm{C} 3, \mathrm{C} 9{ }^{\prime}-\mathrm{C} 7, \mathrm{C} 9{ }^{\prime}-\mathrm{C} 4, \mathrm{C} 2^{\prime}-\mathrm{C} 3, \mathrm{O} 8^{\prime}-\mathrm{C} 9, \mathrm{C} 7^{\prime} \mathrm{a}-\mathrm{C} 5$

8

6

[a] Interactions listed are those involving any heavy atom of the rotator that resides within the nominal van der Waals distance (taken from Ref. 5) of any heavy atom of the stator. [b] Total number of possible interactions. 


\section{References for this document}

[1] B. O. Roos, P. R. Taylor, P. E. M. Siegbahn, Chem. Phys. 1980, 48, 157-173.

[2] K. Andersson, P.-Å. Malmqvist, B. O. Roos, A. J. Sadlej, K. Wolinski, J. Phys. Chem. 1990, 94, 5483-5488.

[3] K. Andersson, P.-Å. Malmqvist, B. O. Roos, J. Chem. Phys. 1992, 96, 1218-1226.

[4] J. Finley, P.-Å. Malmqvist, B. O. Roos, L. Serrano-Andrés, Chem. Phys. Lett. 1998, 288, 299-306.

[5] A. Bondi, J. Phys. Chem. 1964, 68, 441-451. 


\section{Cartesian coordinates for stationary points of motors $2 a-2 c$}

\section{CASSCF geometries of stationary points relevant for the photoisomerizations of motors $2 a-2 c$}

$\begin{array}{lrrr}\text { motor 2a-anti-(M)-stable- } \boldsymbol{E} & \\ & & & \\ \mathrm{C} & -1.80655483 & 2.50038437 & 0.19913850 \\ \mathrm{C} & -1.18814040 & 0.13480678 & 0.18124522 \\ \mathrm{C} & -3.29995827 & 0.81649868 & -0.80807371 \\ \mathrm{C} & -2.60825665 & -0.18836433 & -0.22843553 \\ \mathrm{C} & -0.11703182 & -0.67629595 & -0.06062023 \\ \mathrm{C} & 1.34920601 & -0.39083917 & 0.05451239 \\ \mathrm{C} & -0.17702135 & -2.15928985 & -0.45298752 \\ \mathrm{C} & 2.03982224 & -1.59607372 & 0.24856870 \\ \mathrm{H} & -1.06022488 & -2.64000537 & -0.05356154 \\ \mathrm{C} & 1.07410265 & -2.75302109 & 0.22907573 \\ \mathrm{H} & 1.47171813 & -3.62011828 & -0.30118892 \\ \mathrm{C} & 2.09427874 & 0.79042234 & -0.14671296 \\ \mathrm{C} & 3.48769450 & 0.74936719 & -0.01822691 \\ \mathrm{C} & 4.14731252 & -0.45576866 & 0.25698656 \\ \mathrm{C} & 3.42935730 & -1.64418565 & 0.37358557 \\ \mathrm{H} & 0.83843173 & -3.07587149 & 1.24740591 \\ \mathrm{H} & 5.22596321 & -0.45803339 & 0.34523696 \\ \mathrm{H} & 3.93918120 & -2.58504959 & 0.53544459 \\ \mathrm{H} & 4.07390886 & 1.64512813 & -0.15403572 \\ \mathrm{C} & -1.07375852 & 1.51904156 & 0.75669909 \\ \mathrm{C} & -3.30651333 & -1.50912259 & 0.04678631 \\ \mathrm{H} & -2.90391597 & -2.31313847 & -0.56840813 \\ \mathrm{C} & -0.29795677 & 1.72950819 & 2.03349459 \\ \mathrm{H} & 0.71003464 & 1.32652372 & 1.99322048 \\ \mathrm{H} & -0.23097310 & 2.79116143 & 2.27364281 \\ \mathrm{H} & -0.80860918 & 1.23214102 & 2.86283680 \\ \mathrm{H} & -1.76831267 & 3.50876170 & 0.59562490 \\ \mathrm{C} & -0.15724242 & -2.35679281 & -1.97206985 \\ \mathrm{H} & -0.17848492 & -3.41985787 & -2.22395233 \\ \mathrm{H} & -1.02112156 & -1.88106780 & -2.43866344 \\ \mathrm{H} & 0.74084474 & -1.92373030 & -2.41693169 \\ \mathrm{O} & 1.42188773 & 1.90277031 & -0.49048106 \\ \mathrm{C} & 2.07230521 & 3.11550795 & -0.68332916 \\ \mathrm{H} & 2.59269320 & 3.44652894 & 0.21857092 \\ \mathrm{H} & 2.78704389 & 3.06670216 & -1.50810261 \\ \mathrm{H} & 1.30388036 & 3.84337204 & -0.92981782 \\ \mathrm{C} & -2.74090110 & 2.21004981 & -0.94613093 \\ \mathrm{H} & -3.55879735 & 2.93329456 & -0.95615501 \\ \mathrm{H} & -2.22537812 & 2.32533813 & -1.90751832 \\ \mathrm{H} & -4.31520897 & 0.64414885 & -1.14675535 \\ \mathrm{C} & -3.28626949 & -1.92050507 & 1.51923442 \\ \mathrm{H} & -3.75656887 & -1.15582396 & 2.14064630 \\ \mathrm{H} & -3.83195261 & -2.85493885 & 1.66699198 \\ \mathrm{H} & -2.27028919 & -2.06493989 & 1.88953737 \\ \mathrm{H} & -4.34511555 & -1.39849371 & -0.26820535\end{array}$

motor 2a - anti-(M)-stable- $Z$

$\begin{array}{rrrr}\mathrm{C} & 2.42990384 & -1.82085433 & -0.14163690 \\ \mathrm{C} & 1.25694549 & 0.30703867 & 0.15312587 \\ \mathrm{C} & 3.50518700 & 0.30462258 & -0.78094114 \\ \mathrm{C} & 2.56304834 & 1.01249587 & -0.12194954 \\ \mathrm{C} & 0.03047801 & 0.88602489 & -0.00619804 \\ \mathrm{C} & -1.33212798 & 0.26464726 & -0.00856591\end{array}$




$\begin{array}{lrrr}\mathrm{C} & -0.24761945 & 2.38906860 & -0.15352247 \\ \mathrm{C} & -2.28195661 & 1.23401738 & 0.34480870 \\ \mathrm{H} & 0.50087872 & 2.98375078 & 0.35341438 \\ \mathrm{C} & -1.60509789 & 2.56347207 & 0.56080807 \\ \mathrm{H} & -2.18610866 & 3.39787336 & 0.16416323 \\ \mathrm{C} & -1.78366308 & -1.00230257 & -0.43463297 \\ \mathrm{C} & -3.15057307 & -1.29688996 & -0.37057357 \\ \mathrm{C} & -4.07176379 & -0.33422915 & 0.06351362 \\ \mathrm{C} & -3.64686458 & 0.94747525 & 0.40732643 \\ \mathrm{H} & -1.45465255 & 2.75095703 & 1.62804593 \\ \mathrm{H} & -5.12313108 & -0.58869672 & 0.09761007 \\ \mathrm{H} & -4.36071406 & 1.70847519 & 0.69479797 \\ \mathrm{H} & -3.51444739 & -2.26518142 & -0.67799286 \\ \mathrm{C} & 1.46035671 & -1.14182580 & 0.49987711 \\ \mathrm{H} & 4.45254594 & 0.77153837 & -1.02623446 \\ \mathrm{C} & 2.87612014 & 2.42228995 & 0.33455850 \\ \mathrm{H} & 2.45509546 & 3.18585764 & -0.31902456 \\ \mathrm{H} & 2.50577856 & 2.60525150 & 1.34429383 \\ \mathrm{H} & 3.95577815 & 2.56896565 & 0.34830019 \\ \mathrm{C} & 0.72585865 & -1.74602339 & 1.67912979 \\ \mathrm{H} & -0.33112735 & -1.49420737 & 1.65387087 \\ \mathrm{C} & -0.30894253 & 2.82407866 & -1.62144005 \\ \mathrm{H} & -1.08604607 & 2.28188177 & -2.16373350 \\ \mathrm{H} & -0.52701122 & 3.89196255 & -1.69944451 \\ \mathrm{H} & 0.63980541 & 2.63465851 & -2.12573327 \\ \mathrm{O} & -0.86879690 & -1.86105516 & -0.91756248 \\ \mathrm{C} & -1.22196177 & -3.13586320 & -1.34318292 \\ \mathrm{H} & -0.30402622 & -3.62044642 & -1.66505455 \\ \mathrm{H} & -1.66624881 & -3.72561784 & -0.53789627 \\ \mathrm{H} & -1.91667732 & -3.10474900 & -2.18575328 \\ \mathrm{C} & 3.31982489 & -1.14465312 & -1.15037871 \\ \mathrm{H} & 2.90229750 & -1.23381622 & -2.16092693 \\ \mathrm{H} & 4.29154187 & -1.64176685 & -1.18398267 \\ \mathrm{H} & 0.78662760 & -2.83421044 & 1.60940890 \\ \mathrm{C} & 1.30377238 & -1.29496532 & 3.02284206 \\ \mathrm{H} & 0.75068465 & -1.74172756 & 3.85232695 \\ \mathrm{H} & 2.35142981 & -1.58604601 & 3.12013649 \\ \mathrm{H} & 1.24775773 & -0.21021217 & 3.13473255 \\ \mathrm{H} & 2.61706853 & -2.86227851 & 0.09612803\end{array}$

motor $2 \mathrm{a}$ - anti-(M)-stable- $E^{*}$

$\begin{array}{rrrr}\text { C } & -2.99102396 & -0.81751753 & -1.75679703 \\ \text { C } & -1.15070918 & 0.11912722 & -0.36761600 \\ \text { C } & -3.24317584 & -0.66718306 & 0.71909237 \\ \text { C } & -1.92744648 & -0.06680691 & 0.80817820 \\ \text { C } & 0.20483560 & 0.74785893 & -0.29020328 \\ \text { C } & 1.45082548 & 0.10499933 & -0.08348880 \\ \text { C } & 0.45126855 & 2.20507288 & -0.67497857 \\ \text { C } & 2.51858254 & 1.03954594 & -0.18494808 \\ \text { H } & 0.26955199 & 2.29622415 & -1.75181907 \\ \text { C } & 1.96102849 & 2.42482192 & -0.40944852 \\ \text { H } & 2.11068803 & 3.03666357 & 0.48499532 \\ \text { C } & 1.75564591 & -1.26292287 & 0.19025083 \\ \text { C } & 3.08557393 & -1.64470163 & 0.33481400 \\ \text { C } & 4.12607059 & -0.69940239 & 0.21889467 \\ \text { C } & 3.84377485 & 0.64915288 & -0.03748951 \\ \text { H } & 2.45493406 & 2.94537279 & -1.23196588 \\ \text { H } & 5.15002973 & -1.02882370 & 0.33383126 \\ \text { H } & 4.64720924 & 1.37036655 & -0.11701748 \\ \text { H } & 3.34095388 & -2.67404147 & 0.53634451 \\ \text { C } & -1.65316783 & -0.27936019 & -1.63365662 \\ \text { C } & -1.40513208 & 0.23408383 & 2.19747029 \\ \text { H } & -1.37257307 & -0.70412736 & 2.76169089 \\ \text { H } & -0.37556445 & 0.58060220 & 2.13984566 \\ \text { C } & -0.85532320 & -0.14815100 & -2.90820692\end{array}$




$\begin{array}{crrc}\mathrm{H} & 0.21488353 & -0.09142238 & -2.72315889 \\ \mathrm{H} & -1.03305663 & -1.00822007 & -3.55652399 \\ \mathrm{H} & -1.14573848 & 0.74357240 & -3.47313991 \\ \mathrm{H} & -3.36067503 & -1.08004219 & -2.73915202 \\ \mathrm{C} & -0.44539518 & 3.22751185 & 0.02032998 \\ \mathrm{H} & -0.21465173 & 4.23633935 & -0.32990003 \\ \mathrm{H} & -1.49945846 & 3.03701003 & -0.18519213 \\ \mathrm{H} & -0.30195314 & 3.20940725 & 1.10239026 \\ \mathrm{O} & 0.70792854 & -2.10354730 & 0.29105306 \\ \mathrm{C} & 0.89956715 & -3.45716844 & 0.54212246 \\ \mathrm{H} & 1.48408832 & -3.93715335 & -0.24642833 \\ \mathrm{H} & 1.39328711 & -3.62608006 & 1.50214252 \\ \mathrm{H} & -0.08694568 & -3.91213423 & 0.57178839 \\ \mathrm{H} & -3.79812797 & -0.85336263 & 1.62827353 \\ \mathrm{C} & -2.23148652 & 1.25367162 & 2.98621210 \\ \mathrm{H} & -3.25699257 & 0.91544200 & 3.14249561 \\ \mathrm{H} & -1.78936758 & 1.42385022 & 3.97044520 \\ \mathrm{H} & -2.27728544 & 2.21334974 & 2.46918696 \\ \mathrm{C} & -3.90120312 & -1.00130646 & -0.58339389 \\ \mathrm{H} & -4.80205776 & -0.38582771 & -0.71225122 \\ \mathrm{H} & -4.26770815 & -2.03467371 & -0.55758689\end{array}$

motor 2 a - anti-(M)-stable- $Z^{*}$

$\begin{array}{lrrc}\mathrm{C} & 3.18162496 & -0.05654587 & -1.57780429 \\ \mathrm{C} & 1.21075327 & 0.45625000 & -0.14711505 \\ \mathrm{C} & 3.38472278 & -0.13754226 & 0.90549737 \\ \mathrm{C} & 1.98400284 & 0.21288471 & 1.01850203 \\ \mathrm{C} & -0.21114442 & 0.89956194 & -0.01116307 \\ \mathrm{C} & -1.38375762 & 0.10816093 & 0.01521429 \\ \mathrm{C} & -0.57304843 & 2.35664102 & 0.27130738 \\ \mathrm{C} & -2.53153950 & 0.92161116 & 0.23132597 \\ \mathrm{H} & -0.18793478 & 2.61049220 & 1.26481657 \\ \mathrm{C} & -2.12271318 & 2.37346614 & 0.31135555 \\ \mathrm{H} & -2.52902420 & 2.92788148 & -0.53906467 \\ \mathrm{C} & -1.55924266 & -1.30083484 & -0.14341429 \\ \mathrm{C} & -2.83979888 & -1.83784768 & -0.06937967 \\ \mathrm{C} & -3.95945768 & -1.00956156 & 0.15615523 \\ \mathrm{C} & -3.80694156 & 0.37573317 & 0.30415283 \\ \mathrm{H} & -2.50541789 & 2.85822709 & 1.21156755 \\ \mathrm{H} & -4.94227138 & -1.45812648 & 0.21099067 \\ \mathrm{H} & -4.67153714 & 1.00594419 & 0.46953394 \\ \mathrm{H} & -2.99545407 & -2.90018056 & -0.18168528 \\ \mathrm{C} & 1.78942600 & 0.31601953 & -1.43632931 \\ \mathrm{C} & 1.39432409 & 0.21154988 & 2.41314564 \\ \mathrm{H} & 2.06735543 & 0.75517629 & 3.08324346 \\ \mathrm{H} & 0.44889150 & 0.74957307 & 2.42829720 \\ \mathrm{C} & 1.00170977 & 0.45544358 & -2.71424904 \\ \mathrm{H} & -0.02121495 & 0.77828560 & -2.54295533 \\ \mathrm{H} & 1.47542088 & 1.17303710 & -3.38956290 \\ \mathrm{H} & 0.95959580 & -0.50252076 & -3.24042025 \\ \mathrm{H} & 3.59701369 & -0.15100246 & -2.57258842 \\ \mathrm{C} & 0.01983584 & 3.37229713 & -0.70641859 \\ \mathrm{H} & -0.26580467 & 4.38664295 & -0.41817198 \\ \mathrm{H} & 1.10932255 & 3.32410138 & -0.71786023 \\ \mathrm{H} & -0.33743705 & 3.20222036 & -1.72391965 \\ \mathrm{O} & -0.44200937 & -2.02509618 & -0.35369971 \\ \mathrm{C} & -0.51035727 & -3.39718389 & -0.56824206 \\ \mathrm{H} & -0.91977348 & -3.92360168 & 0.29716530 \\ \mathrm{H} & -1.11136241 & -3.63951090 & -1.44790125 \\ \mathrm{H} & 0.50789821 & -3.73849295 & -0.73462484 \\ \mathrm{H} & 3.96155432 & -0.27921692 & 1.80996342 \\ \mathrm{C} & 1.16369774 & -1.19643988 & 2.97098896 \\ \mathrm{H} & 2.09778297 & -1.75753202 & 3.03889963 \\ \mathrm{H} & 0.72927185 & -1.14987111 & 3.97225015 \\ \mathrm{H} & 0.48467996 & -1.75745576 & 2.32879150\end{array}$


$\begin{array}{cccc}\mathrm{C} & 4.09903802 & -0.22577109 & -0.40721354 \\ \mathrm{H} & 4.62188309 & -1.18704694 & -0.48097988 \\ \mathrm{H} & 4.89396803 & 0.53142591 & -0.44730032\end{array}$

motor 2a - (P)-unstable- $Z$

$\begin{array}{llll}\text { C } & -3.24258246 & -0.38187910 & -1.63767214\end{array}$

$\begin{array}{llll}\text { C } & -1.13559469 & 0.15820421 & -0.45520730\end{array}$

$\begin{array}{llll}\text { C } & -2.92070572 & -1.11269805 & 0.70663621\end{array}$

$\begin{array}{llll}\text { C } & -1.70068560 & -0.52952801 & 0.74475386\end{array}$

$\begin{array}{llll}\text { C } & 0.15381314 & 0.67921816 & -0.47688532\end{array}$

$\begin{array}{llll}\text { C } & 1.37753396 & 0.11903356 & 0.14466916\end{array}$

$\begin{array}{llll}\text { C } & 0.57567957 & 2.00699351 & -1.14397287\end{array}$

$\begin{array}{llll}\text { C } & 2.42905259 & 1.04211560 & 0.08258379\end{array}$

$\begin{array}{llll}\mathrm{H} & 0.71310517 & 1.87739992 & -2.21754737\end{array}$

$\begin{array}{llll}\text { C } & 1.96007071 & 2.33331937 & -0.52723059\end{array}$

$\begin{array}{llll}\mathrm{H} & 1.86663813 & 3.10672130 & 0.24012384\end{array}$

$\begin{array}{llll}\text { C } & 1.66668043 & -1.21032774 & 0.53917135\end{array}$

$\begin{array}{llll}\text { C } & 2.95827910 & -1.53308534 & 0.96201989\end{array}$

$\begin{array}{llll}\text { C } & 3.97664675 & -0.56751084 & 0.95722454\end{array}$

$\begin{array}{llll}\text { C } & 3.72343320 & 0.72334768 & 0.50141581\end{array}$

$\begin{array}{llll}\mathrm{H} & 2.66275140 & 2.71301049 & -1.27091797\end{array}$

$\begin{array}{llll}\mathrm{H} & 4.96940947 & -0.84676357 & 1.28508196\end{array}$

$\begin{array}{llll}\mathrm{H} & 4.51647621 & 1.45833525 & 0.45401815\end{array}$

$\begin{array}{llll}\mathrm{H} & 3.19331744 & -2.53716273 & 1.28000496\end{array}$

$\begin{array}{llll}\text { C } & -2.04247032 & 0.24790682 & -1.64076198\end{array}$

$\begin{array}{llll}\text { C } & -1.08995587 & -0.32995138 & 2.12641149\end{array}$

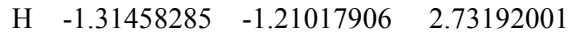

$\begin{array}{llll}\mathrm{H} & -0.01123281 & -0.25921370 & 2.09275293\end{array}$

C $\quad-1.55089292 \quad 0.76506912 \quad-2.98071830$

$\begin{array}{llll}\mathrm{H} & -0.60959375 & 0.28648611 & -3.25416937\end{array}$

$\begin{array}{llll}\mathrm{H} & -2.27755193 & 0.52339284 & -3.75454146\end{array}$

$\begin{array}{llll}\mathrm{H} & -1.39631789 & 1.84001036 & -3.01181714\end{array}$

$\begin{array}{llll}\mathrm{H} & -3.81722498 & -0.40117362 & -2.55635522\end{array}$

C $\quad-0.40118193 \quad 3.16003389 \quad-0.89233648$

$\begin{array}{llll}\mathrm{H} & -0.03959842 & 4.07371065 & -1.37122590\end{array}$

$\begin{array}{llll}\mathrm{H} & -1.40368835 & 2.96220568 & -1.26340664\end{array}$

$\begin{array}{llll}\mathrm{H} & -0.48481726 & 3.35723477 & 0.17802090\end{array}$

$\begin{array}{llll}\text { O } & 0.66836355 & -2.10856692 & 0.46533315\end{array}$

$\begin{array}{llll}\text { C } & 0.87067640 & -3.44671742 & 0.78294256\end{array}$

$\begin{array}{llll}\mathrm{H} & 1.62868784 & -3.90475308 & 0.14341700\end{array}$

$\begin{array}{llll}\mathrm{H} & 1.16092852 & -3.57723132 & 1.82805246\end{array}$

$\begin{array}{llll}\mathrm{H} & -0.07783143 & -3.95098843 & 0.61884535\end{array}$

$\begin{array}{llll}\mathrm{H} & -3.33264284 & -1.51026904 & 1.62820118\end{array}$

$\begin{array}{llll}\text { C } & -1.63231688 & 0.91498134 & 2.83362730\end{array}$

$\begin{array}{llll}\mathrm{H} & -2.71077537 & 0.84822625 & 2.98821898\end{array}$

$\begin{array}{llll}\mathrm{H} & -1.15981226 & 1.04047782 & 3.81089424\end{array}$

$\begin{array}{llll}\mathrm{H} & -1.43743527 & 1.81806310 & 2.25238330\end{array}$

$\begin{array}{llll}\text { C } & -3.80982047 & -1.15449342 & -0.49219070\end{array}$

$\begin{array}{llll}\mathrm{H} & -4.79868441 & -0.76343712 & -0.22578934\end{array}$

H $\quad-3.98903892 \quad-2.18949791 \quad-0.80849325$

motor 2a - (P)-unstable- $E$

$\begin{array}{lrrr}\mathrm{C} & 2.91028285 & -0.16411549 & -1.72559822 \\ \mathrm{C} & 1.13162806 & 0.37885725 & -0.11183917 \\ \mathrm{C} & 3.51403913 & 0.62398578 & 0.54704678 \\ \mathrm{C} & 2.20726830 & 0.66171876 & 0.88964801 \\ \mathrm{C} & -0.18650347 & 0.71426945 & 0.14913250 \\ \mathrm{C} & -1.42440780 & 0.03134129 & -0.30020832 \\ \mathrm{C} & -0.68045967 & 1.98529561 & 0.87344144 \\ \mathrm{C} & -2.50236366 & 0.92551024 & -0.28846988 \\ \mathrm{H} & -0.93648302 & 1.75211808 & 1.91194494 \\ \mathrm{C} & -2.01415789 & 2.28303297 & 0.13927511 \\ \mathrm{H} & -1.82752192 & 2.91832945 & -0.73220628 \\ \mathrm{C} & -1.67938215 & -1.34728917 & -0.45490982\end{array}$




$\begin{array}{crrc}\mathrm{C} & -2.98215225 & -1.76778381 & -0.74304314 \\ \mathrm{C} & -4.03116230 & -0.84009916 & -0.82179793 \\ \mathrm{C} & -3.80504075 & 0.51358801 & -0.57249183 \\ \mathrm{H} & -2.72663068 & 2.80971590 & 0.77605869 \\ \mathrm{H} & -5.02983173 & -1.19370429 & -1.04286073 \\ \mathrm{H} & -4.62499445 & 1.21996247 & -0.57466581 \\ \mathrm{H} & -3.20111341 & -2.81509999 & -0.88467582 \\ \mathrm{C} & 1.59055381 & -0.18399058 & -1.41686225 \\ \mathrm{C} & 1.86836306 & 0.66468089 & 2.37443333 \\ \mathrm{H} & 2.69172204 & 1.13374152 & 2.91708089 \\ \mathrm{H} & 0.98860618 & 1.26021110 & 2.59295475 \\ \mathrm{C} & 0.62539579 & -0.51961156 & -2.53511843 \\ \mathrm{H} & -0.10170919 & 0.27899637 & -2.68283439 \\ \mathrm{H} & 1.17463946 & -0.63944097 & -3.46807163 \\ \mathrm{H} & 0.07902418 & -1.43819166 & -2.35217865 \\ \mathrm{H} & 3.20864605 & -0.44837373 & -2.72802061 \\ \mathrm{C} & 0.23807180 & 3.20548131 & 0.83512997 \\ \mathrm{H} & -0.28833537 & 4.07333163 & 1.23998031 \\ \mathrm{H} & 1.15094838 & 3.07962333 & 1.41111690 \\ \mathrm{H} & 0.52488280 & 3.43774751 & -0.19215240 \\ \mathrm{O} & -0.64633811 & -2.19640041 & -0.27561166 \\ \mathrm{C} & -0.83659723 & -3.57441700 & -0.29174788 \\ \mathrm{H} & -1.55578974 & -3.89231530 & 0.46643953 \\ \mathrm{H} & -1.17205290 & -3.92757121 & -1.26975804 \\ \mathrm{H} & 0.12765506 & -4.02441308 & -0.07149151 \\ \mathrm{H} & 4.26262797 & 0.73800361 & 1.32358973 \\ \mathrm{C} & 1.64725982 & -0.74842222 & 2.92145066 \\ \mathrm{H} & 2.55001212 & -1.35358627 & 2.81863287 \\ \mathrm{H} & 1.38324722 & -0.71539083 & 3.98120649 \\ \mathrm{H} & 0.84338292 & -1.25720402 & 2.38799293 \\ \mathrm{C} & 4.00725008 & 0.31975802 & -0.82996573 \\ \mathrm{H} & 4.80184957 & -0.43208662 & -0.77045948 \\ \mathrm{H} & 4.48220206 & 1.20145181 & -1.27841519\end{array}$

motor $2 \mathrm{~b}$ - anti-(M)-stable- $E$

$\begin{array}{lrrc}\mathrm{C} & -1.91592790 & 2.44979105 & 0.27244668 \\ \mathrm{C} & -1.16299175 & 0.13856288 & 0.15584247 \\ \mathrm{C} & -3.29150868 & 0.90209811 & -0.70663060 \\ \mathrm{C} & -2.57097750 & -0.15465420 & -0.27627244 \\ \mathrm{C} & -0.08645008 & -0.68001834 & -0.03804358 \\ \mathrm{C} & 1.36992465 & -0.36940757 & 0.09069641 \\ \mathrm{C} & -0.11599563 & -2.18098423 & -0.35876612 \\ \mathrm{C} & 2.07336435 & -1.55022473 & 0.36912628 \\ \mathrm{H} & -1.00858790 & -2.65619459 & 0.02882064 \\ \mathrm{C} & 1.11695903 & -2.71581430 & 0.40436592 \\ \mathrm{H} & 1.53558531 & -3.61561527 & -0.04962042 \\ \mathrm{C} & 2.09689246 & 0.80499477 & -0.19189186 \\ \mathrm{C} & 3.49011018 & 0.79404816 & -0.05260001 \\ \mathrm{C} & 4.16353270 & -0.37988316 & 0.31057452 \\ \mathrm{C} & 3.46208416 & -1.56914393 & 0.50515009 \\ \mathrm{H} & 0.84943731 & -2.96554901 & 1.43532425 \\ \mathrm{H} & 5.24149021 & -0.36068490 & 0.40499532 \\ \mathrm{H} & 3.98569588 & -2.48877013 & 0.73201097 \\ \mathrm{H} & 4.06513381 & 1.68636908 & -0.24701972 \\ \mathrm{C} & -1.07489845 & 1.51258444 & 0.74155144 \\ \mathrm{C} & -3.26610926 & -1.50149163 & -0.23132766 \\ \mathrm{H} & -2.75223572 & -2.23698462 & -0.84686935 \\ \mathrm{C} & -0.23866884 & 1.80261656 & 1.96289460 \\ \mathrm{H} & 0.80609981 & 1.53680574 & 1.84558453 \\ \mathrm{H} & -0.28931548 & 2.86201784 & 2.21792385 \\ \mathrm{H} & -0.62333454 & 1.23890071 & 2.81665376 \\ \mathrm{H} & -1.94968164 & 3.47163146 & 0.62026408 \\ \mathrm{C} & -0.02071741 & -2.45899251 & -1.86279153 \\ \mathrm{H} & -0.02865449 & -3.53408845 & -2.05822085 \\ \mathrm{H} & -0.85776898 & -2.01104926 & -2.40005435\end{array}$




$\begin{array}{rrrr}\mathrm{H} & 0.89868731 & -2.04779449 & -2.28363943 \\ \mathrm{O} & 1.40511359 & 1.87934838 & -0.61281520 \\ \mathrm{C} & 2.04508348 & 3.06203414 & -0.96511246 \\ \mathrm{H} & 2.57770667 & 3.50477990 & -0.11995687 \\ \mathrm{H} & 2.74710821 & 2.91329308 & -1.78867216 \\ \mathrm{H} & 1.26868852 & 3.75057493 & -1.28772537 \\ \mathrm{H} & -4.28990507 & 0.81919195 & -1.10977051 \\ \mathrm{C} & -3.45225733 & -2.04144886 & 1.18763059 \\ \mathrm{H} & -4.05818542 & -1.35637776 & 1.78341351 \\ \mathrm{H} & -3.95644056 & -3.01013588 & 1.17122643 \\ \mathrm{H} & -2.49942656 & -2.16859839 & 1.70362185 \\ \mathrm{H} & -4.25084179 & -1.38131766 & -0.68661497 \\ \mathrm{O} & -2.86810769 & 2.18232869 & -0.65303876\end{array}$

motor $2 \mathrm{~b}$ - anti-(M)-stable- $Z$

$\begin{array}{lrrr}\mathrm{C} & 2.51636833 & -1.75315213 & 0.05811284 \\ \mathrm{C} & 1.23450819 & 0.31706358 & 0.12998321 \\ \mathrm{C} & 3.54042999 & 0.18481397 & -0.60607441 \\ \mathrm{C} & 2.55115916 & 0.97825270 & -0.14588334 \\ \mathrm{C} & 0.00924730 & 0.89774842 & -0.04100465 \\ \mathrm{C} & -1.34279032 & 0.26190425 & -0.05437327 \\ \mathrm{C} & -0.29015744 & 2.39901856 & -0.16285885 \\ \mathrm{C} & -2.30426507 & 1.20795029 & 0.32856261 \\ \mathrm{H} & 0.46090108 & 2.99998264 & 0.33533155 \\ \mathrm{C} & -1.63556110 & 2.53546212 & 0.58592308 \\ \mathrm{H} & -2.22986002 & 3.38033512 & 0.23380250 \\ \mathrm{C} & -1.77293086 & -0.99046078 & -0.53761533 \\ \mathrm{C} & -3.13679247 & -1.30394998 & -0.49726235 \\ \mathrm{C} & -4.07176958 & -0.36946060 & -0.03169648 \\ \mathrm{C} & -3.66565103 & 0.90329226 & 0.36774470 \\ \mathrm{H} & -1.46404195 & 2.68121754 & 1.65650064 \\ \mathrm{H} & -5.12040044 & -0.63676152 & -0.01709095 \\ \mathrm{H} & -4.39222321 & 1.64331705 & 0.67726776 \\ \mathrm{H} & -3.48732043 & -2.26311408 & -0.84634816 \\ \mathrm{C} & 1.44000366 & -1.10754047 & 0.54021881 \\ \mathrm{H} & 4.51015579 & 0.55370972 & -0.90536518 \\ \mathrm{C} & 2.86110207 & 2.44612011 & 0.03999332 \\ \mathrm{H} & 2.35055637 & 3.08462835 & -0.67849512 \\ \mathrm{H} & 2.58933283 & 2.78242269 & 1.04155241 \\ \mathrm{H} & 3.93142656 & 2.61391377 & -0.07873993 \\ \mathrm{C} & 0.62716317 & -1.75891845 & 1.63996859 \\ \mathrm{H} & -0.43712209 & -1.62547044 & 1.47851352 \\ \mathrm{C} & -0.40688783 & 2.85653506 & -1.62111713 \\ \mathrm{H} & -1.20293986 & 2.32033576 & -2.14096825 \\ \mathrm{H} & -0.63113488 & 3.92471565 & -1.67388847 \\ \mathrm{H} & 0.51991838 & 2.67785525 & -2.16765102 \\ \mathrm{O} & -0.83791518 & -1.81679239 & -1.04057520 \\ \mathrm{C} & -1.17720245 & -3.05127805 & -1.58223468 \\ \mathrm{H} & -0.25188725 & -3.50195141 & -1.93100318 \\ \mathrm{H} & -1.63085617 & -3.71153396 & -0.83916522 \\ \mathrm{H} & -1.85905286 & -2.94845857 & -2.42945193 \\ \mathrm{H} & 0.80674829 & -2.83624195 & 1.61148771 \\ \mathrm{C} & 0.98421247 & -1.22788087 & 3.03016404 \\ \mathrm{H} & 0.37856151 & -1.71601763 & 3.79707567 \\ \mathrm{H} & 2.03517026 & -1.40738553 & 3.26488884 \\ \mathrm{H} & 0.80534472 & -0.15340220 & 3.10148392 \\ \mathrm{H} & 2.76582297 & -2.77824318 & 0.28955803 \\ \mathrm{O} & 3.44784738 & -1.15628765 & -0.72150164\end{array}$

motor $2 \mathrm{~b}$ - anti-(M)-stable- $E^{*}$
$\begin{array}{llll}\text { C } & -3.01070870 & -0.74169055 & -1.69581549\end{array}$
$\begin{array}{llll}\text { C } & -1.13837071 & 0.08295717 & -0.36921413\end{array}$
$\begin{array}{llll}\text { C } & -3.23024978 & -0.68544819 & 0.60965210\end{array}$
$\begin{array}{llll}\text { C } & -1.91743347 & -0.11446064 & 0.79373502\end{array}$ 


$\begin{array}{lrrr}\mathrm{C} & 0.20107839 & 0.74031616 & -0.28066285 \\ \mathrm{C} & 1.45948178 & 0.12733618 & -0.06883378 \\ \mathrm{C} & 0.41029223 & 2.20137412 & -0.67220342 \\ \mathrm{C} & 2.50379782 & 1.08854681 & -0.16633243 \\ \mathrm{H} & 0.24077451 & 2.28278310 & -1.75142189 \\ \mathrm{C} & 1.91123762 & 2.45935171 & -0.38967595 \\ \mathrm{H} & 2.03439693 & 3.06939909 & 0.50995898 \\ \mathrm{C} & 1.79438315 & -1.23302101 & 0.20436239 \\ \mathrm{C} & 3.13238877 & -1.58358665 & 0.35244764 \\ \mathrm{C} & 4.15001112 & -0.61319201 & 0.24057817 \\ \mathrm{C} & 3.83704992 & 0.72911441 & -0.01494027 \\ \mathrm{H} & 2.39842891 & 2.99819731 & -1.20419637 \\ \mathrm{H} & 5.18118270 & -0.91818255 & 0.35804826 \\ \mathrm{H} & 4.62375397 & 1.46881117 & -0.09088617 \\ \mathrm{H} & 3.41136226 & -2.60686709 & 0.55356244 \\ \mathrm{C} & -1.64364791 & -0.27601689 & -1.63692065 \\ \mathrm{C} & -1.43765274 & 0.09881606 & 2.21003464 \\ \mathrm{H} & -1.45479633 & -0.86263506 & 2.73593091 \\ \mathrm{H} & -0.39402586 & 0.40742369 & 2.19373680 \\ \mathrm{C} & -0.89697201 & -0.13559876 & -2.93596941 \\ \mathrm{H} & 0.18024918 & -0.12979227 & -2.78011781 \\ \mathrm{H} & -1.12535806 & -0.97267591 & -3.60007419 \\ \mathrm{H} & -1.16090123 & 0.78096305 & -3.47500139 \\ \mathrm{H} & -3.54593852 & -1.00195327 & -2.59274566 \\ \mathrm{C} & -0.52014949 & 3.20266645 & 0.00902059 \\ \mathrm{H} & -0.30798829 & 4.21610530 & -0.33949404 \\ \mathrm{H} & -1.56650084 & 2.98737962 & -0.21046353 \\ \mathrm{H} & -0.39102221 & 3.18786617 & 1.09287453 \\ \mathrm{O} & 0.76434318 & -2.09464025 & 0.30154418 \\ \mathrm{C} & 0.98263416 & -3.44660692 & 0.54239255 \\ \mathrm{H} & 1.57849230 & -3.90760749 & -0.24886000 \\ \mathrm{H} & 1.47756621 & -3.61250184 & 1.50219986 \\ \mathrm{H} & 0.00545203 & -3.92136906 & 0.56607738 \\ \mathrm{H} & -3.94874935 & -0.86159876 & 1.39140414 \\ \mathrm{C} & -2.24795835 & 1.11386486 & 3.02133167 \\ \mathrm{H} & -3.29378276 & 0.81550453 & 3.11818001 \\ \mathrm{H} & -1.84196853 & 1.21152566 & 4.03074328 \\ \mathrm{H} & -2.23067499 & 2.09971926 & 2.55444429 \\ \mathrm{O} & -3.70127698 & -0.92588673 & -0.59306139\end{array}$

motor $2 \mathrm{~b}$ - anti-(M)-stable- $Z^{*}$

$\begin{array}{rrrr}\text { C } & 3.30872279 & -0.47646031 & 0.83818231 \\ \text { C } & 1.20980572 & 0.35838589 & -0.08143594 \\ \text { C } & 3.21586495 & 0.01910626 & -1.42274269 \\ \text { C } & 1.82718108 & 0.40518787 & -1.35036903 \\ \text { C } & -0.18241637 & 0.87434290 & 0.08849272 \\ \text { C } & -1.40361687 & 0.17339216 & -0.03686260 \\ \text { C } & -0.45223169 & 2.28519388 & 0.60674953 \\ \text { C } & -2.50074061 & 1.02715856 & 0.26980304 \\ \text { H } & -0.12044206 & 2.32716759 & 1.64980460 \\ \text { C } & -1.99580061 & 2.41903201 & 0.56721974 \\ \text { H } & -2.30444263 & 3.10708328 & -0.22483610 \\ \text { C } & -1.66539794 & -1.18090556 & -0.40737083 \\ \text { C } & -2.98069361 & -1.63039874 & -0.44694091 \\ \text { C } & -4.04965553 & -0.76595538 & -0.12967151 \\ \text { C } & -3.81115883 & 0.56865366 & 0.22695445 \\ \text { H } & -2.39874660 & 2.81513637 & 1.50107234 \\ \text { H } & -5.06149807 & -1.14650785 & -0.16722649 \\ \text { H } & -4.63757741 & 1.22750780 & 0.46106835 \\ \text { H } & -3.20238016 & -2.65145101 & -0.71839721 \\ \text { C } & 1.92814676 & -0.09747539 & 1.04586237 \\ \text { C } & 1.16210261 & 0.83512839 & -2.62961979 \\ \text { H } & 1.33276705 & 0.09868807 & -3.41988038 \\ \text { H } & 0.08655910 & 0.93854837 & -2.50823857 \\ \text { C } & 1.34453424 & -0.32603203 & 2.41981236\end{array}$




$\begin{array}{rrrr}\mathrm{H} & 0.42234325 & 0.24151929 & 2.53188578 \\ \mathrm{H} & 2.03367815 & 0.06809642 & 3.17435339 \\ \mathrm{H} & 4.00029679 & -0.75755644 & 1.61416139 \\ \mathrm{C} & 0.27552035 & 3.40265046 & -0.14037438 \\ \mathrm{H} & 0.03271483 & 4.37252901 & 0.29985613 \\ \mathrm{H} & 1.35751785 & 3.27604701 & -0.08988672 \\ \mathrm{H} & -0.01384560 & 3.43095192 & -1.19262271 \\ \mathrm{O} & -0.59047851 & -1.94186415 & -0.69033932 \\ \mathrm{C} & -0.73793035 & -3.25590152 & -1.12189811 \\ \mathrm{H} & -1.21341962 & -3.88121931 & -0.36269585 \\ \mathrm{H} & -1.31873376 & -3.31453717 & -2.04534986 \\ \mathrm{H} & 0.26210245 & -3.63639744 & -1.31145430 \\ \mathrm{H} & 3.82406949 & 0.04282849 & -2.31083492 \\ \mathrm{H} & 1.55055351 & 1.79187099 & -2.99356173 \\ \mathrm{C} & 1.05686135 & -1.79865538 & 2.72997869 \\ \mathrm{H} & 1.96606543 & -2.40166760 & 2.68059636 \\ \mathrm{H} & 0.63733926 & -1.91077254 & 3.73260576 \\ \mathrm{H} & 0.34695842 & -2.21011063 & 2.01220414 \\ \mathrm{O} & 3.86358538 & -0.37161119 & -0.34876849\end{array}$

motor 2b - (P)-unstable- $Z$

\begin{tabular}{|c|c|c|c|}
\hline $\mathrm{C}$ & -3.22622081 & -0.47082236 & -1.55584402 \\
\hline $\mathrm{C}$ & -1.12699232 & 0.12512699 & -0.44385777 \\
\hline $\mathrm{C}$ & -2.92278556 & -1.16726484 & 0.61182563 \\
\hline $\mathrm{C}$ & -1.70081049 & -0.60383300 & 0.71641189 \\
\hline $\mathrm{C}$ & 0.14525025 & 0.67746567 & -0.45617766 \\
\hline $\mathrm{C}$ & 1.37604340 & 0.14512917 & 0.16987706 \\
\hline $\mathrm{C}$ & 0.53103721 & 2.01964598 & -1.11376308 \\
\hline $\mathrm{C}$ & 2.39968002 & 1.10166829 & 0.13246239 \\
\hline $\mathrm{H}$ & 0.67823199 & 1.89952722 & -2.18857177 \\
\hline$C$ & 1.90080046 & 2.38118840 & -0.48098104 \\
\hline $\mathrm{H}$ & 1.77761356 & 3.15085438 & 0.28585504 \\
\hline $\mathrm{C}$ & 1.70443011 & -1.18135532 & 0.53938164 \\
\hline $\mathrm{C}$ & 2.99757220 & -1.46794444 & 0.98529743 \\
\hline $\mathrm{C}$ & 3.98231229 & -0.46917243 & 1.01609745 \\
\hline $\mathrm{C}$ & 3.69557666 & 0.81897543 & 0.56998911 \\
\hline $\mathrm{H}$ & 2.60144324 & 2.78093902 & -1.21599369 \\
\hline $\mathrm{H}$ & 4.97719552 & -0.71981922 & 1.36030115 \\
\hline $\mathrm{H}$ & 4.46637145 & 1.57839143 & 0.54347805 \\
\hline $\mathrm{H}$ & 3.26094133 & -2.46947029 & 1.28902303 \\
\hline $\mathrm{C}$ & -2.05698725 & 0.20687547 & -1.59903239 \\
\hline $\mathrm{C}$ & -1.14351261 & -0.48464593 & 2.12547511 \\
\hline $\mathrm{H}$ & -1.53676585 & -1.31223182 & 2.72003318 \\
\hline $\mathrm{H}$ & -0.06740670 & -0.59681599 & 2.14072681 \\
\hline $\mathrm{C}$ & -1.64505133 & 0.73741328 & -2.95568153 \\
\hline $\mathrm{H}$ & -0.70777421 & 0.27724999 & -3.27174794 \\
\hline $\mathrm{H}$ & -2.40119591 & 0.48729611 & -3.69928633 \\
\hline $\mathrm{H}$ & -1.51109300 & 1.81521433 & -2.98465702 \\
\hline $\mathrm{H}$ & -3.88722134 & -0.55729860 & -2.40409241 \\
\hline $\mathrm{C}$ & -0.47706035 & 3.14624117 & -0.86514383 \\
\hline $\mathrm{H}$ & -0.13991838 & 4.06853048 & -1.34538999 \\
\hline $\mathrm{H}$ & -1.47385156 & 2.92212197 & -1.23656525 \\
\hline $\mathrm{H}$ & -0.56795263 & 3.34362460 & 0.20447122 \\
\hline $\mathrm{O}$ & 0.74268155 & -2.11552317 & 0.41307567 \\
\hline $\mathrm{C}$ & 0.98642481 & -3.45148603 & 0.71062932 \\
\hline $\mathrm{H}$ & 1.78124771 & -3.86723667 & 0.08726540 \\
\hline $\mathrm{H}$ & 1.24872714 & -3.59279819 & 1.76184112 \\
\hline $\mathrm{H}$ & 0.06398211 & -3.98837637 & 0.50609829 \\
\hline $\mathrm{H}$ & -3.43448803 & -1.62918430 & 1.44228450 \\
\hline $\mathrm{C}$ & -1.51419147 & 0.83481125 & 2.80676775 \\
\hline $\mathrm{H}$ & -2.59673282 & 0.94783371 & 2.89067382 \\
\hline $\mathrm{H}$ & -1.09222233 & 0.87812924 & 3.81345395 \\
\hline $\mathrm{H}$ & -1.13273581 & 1.69170850 & 2.25058892 \\
\hline $\mathrm{O}$ & -3.68236221 & -1.16599314 & -0.50123021 \\
\hline
\end{tabular}


motor $2 \mathrm{~b}$ - (P)-unstable- $E$

$\begin{array}{crcc}\mathrm{C} & 3.49098289 & 0.03139889 & 0.53433638 \\ \mathrm{C} & 1.13566541 & 0.31591243 & -0.08487510 \\ \mathrm{C} & 2.90287181 & -0.28089099 & -1.66612400 \\ \mathrm{C} & 1.58519138 & -0.08524724 & -1.44051834 \\ \mathrm{C} & -0.16008096 & 0.70696546 & 0.20821577 \\ \mathrm{C} & -1.42106523 & 0.15881487 & -0.33865800 \\ \mathrm{C} & -0.57132329 & 1.86443348 & 1.13958451 \\ \mathrm{C} & -2.48402293 & 1.03762042 & -0.08894959 \\ \mathrm{H} & -0.70311942 & 1.50514106 & 2.16508769 \\ \mathrm{C} & -1.97056939 & 2.27026090 & 0.60463178 \\ \mathrm{H} & -1.87627963 & 3.09689389 & -0.10580377 \\ \mathrm{C} & -1.71063065 & -1.14706189 & -0.79206901 \\ \mathrm{C} & -3.02873876 & -1.47683547 & -1.12408378 \\ \mathrm{C} & -4.06310716 & -0.54526857 & -0.95354073 \\ \mathrm{C} & -3.80230293 & 0.71319609 & -0.41193888 \\ \mathrm{H} & -2.63466410 & 2.60964932 & 1.40112489 \\ \mathrm{H} & -5.07475775 & -0.82820603 & -1.21371350 \\ \mathrm{H} & -4.60814356 & 1.41105631 & -0.22467114 \\ \mathrm{H} & -3.27022787 & -2.46276308 & -1.49092995 \\ \mathrm{C} & 2.23086426 & 0.32920870 & 0.91935866 \\ \mathrm{C} & 0.69729593 & 0.01035613 & -2.66034158 \\ \mathrm{H} & 1.30263682 & -0.02207072 & -3.56632765 \\ \mathrm{H} & -0.02807774 & -0.79320710 & -2.71850824 \\ \mathrm{C} & 1.97152393 & 0.32819163 & 2.41779580 \\ \mathrm{H} & 1.35033882 & 1.16405323 & 2.72211962 \\ \mathrm{H} & 2.92587385 & 0.47285967 & 2.92849398 \\ \mathrm{H} & 4.31144032 & -0.07442778 & 1.22761924 \\ \mathrm{C} & 0.36566243 & 3.07404661 & 1.13623573 \\ \mathrm{H} & -0.05157281 & 3.87140443 & 1.75642340 \\ \mathrm{H} & 1.36230345 & 2.85360630 & 1.50979791 \\ \mathrm{H} & 0.47676592 & 3.46340424 & 0.12250428 \\ \mathrm{O} & -0.69072918 & -2.02887279 & -0.84194781 \\ \mathrm{C} & -0.91111615 & -3.36969652 & -1.13854527 \\ \mathrm{H} & -1.61059713 & -3.82941349 & -0.43671071 \\ \mathrm{H} & -1.28751849 & -3.50618972 & -2.15520444 \\ \mathrm{H} & 0.05033377 & -3.86918652 & -1.05425501 \\ \mathrm{H} & 3.31537119 & -0.44911216 & -2.64843552 \\ \mathrm{H} & 0.15197504 & 0.95502528 & -2.66176916 \\ \mathrm{C} & 1.33638408 & -0.97276718 & 2.91526845 \\ \mathrm{H} & 1.98790484 & -1.82569438 & 2.71646919 \\ \mathrm{H} & 1.16181608 & -0.92624131 & 3.99260296 \\ \mathrm{H} & 0.38122007 & -1.16563227 & 2.42661762 \\ \mathrm{O} & 3.86830687 & -0.23398714 & -0.72708166\end{array}$

motor $2 \mathrm{c}$ - anti-(M)-stable- $E$

$\begin{array}{lrrr}\mathrm{C} & -1.96242481 & 2.08469356 & 0.31767752 \\ \mathrm{C} & -1.03091407 & -0.18599124 & 0.23532431 \\ \mathrm{~S} & -2.98294102 & 1.72761582 & -1.08034995 \\ \mathrm{C} & -3.26288423 & 0.01756221 & -0.74156874 \\ \mathrm{C} & -2.38261941 & -0.77507978 & -0.09253570 \\ \mathrm{C} & 0.15037576 & -0.77831531 & -0.09455591 \\ \mathrm{C} & 1.55609671 & -0.27354391 & 0.01873316 \\ \mathrm{C} & 0.30306825 & -2.21390196 & -0.61263653 \\ \mathrm{C} & 2.43091197 & -1.36896592 & 0.08214559 \\ \mathrm{H} & -0.47980392 & -2.85776372 & -0.23285491 \\ \mathrm{C} & 1.66032866 & -2.65957883 & -0.03052093 \\ \mathrm{H} & 2.16712471 & -3.39424114 & -0.65850913 \\ \mathrm{C} & 2.10321527 & 1.02479929 & -0.06083354 \\ \mathrm{C} & 3.48954092 & 1.18947508 & 0.04443120 \\ \mathrm{C} & 4.33584275 & 0.08156760 & 0.18043884 \\ \mathrm{C} & 3.81410155 & -1.21022025 & 0.18289104 \\ \mathrm{H} & 1.52607240 & -3.11440044 & 0.95528744 \\ \mathrm{H} & 5.40390522 & 0.23978488 & 0.25386700\end{array}$




$\begin{array}{rrrr}\mathrm{H} & 4.46756848 & -2.07095388 & 0.24078660 \\ \mathrm{H} & 3.92479007 & 2.17588417 & 0.00031663 \\ \mathrm{C} & -1.14514526 & 1.16880370 & 0.86920210 \\ \mathrm{C} & -2.84238243 & -2.15189804 & 0.36696596 \\ \mathrm{C} & -0.46036876 & 1.42220022 & 2.18984411 \\ \mathrm{H} & 0.59353914 & 1.15629783 & 2.16420458 \\ \mathrm{H} & -0.54193052 & 2.47057152 & 2.47747106 \\ \mathrm{H} & -0.92771962 & 0.82233250 & 2.97509107 \\ \mathrm{H} & -2.08501722 & 3.07636851 & 0.73291475 \\ \mathrm{C} & 0.27839056 & -2.27155409 & -2.14353559 \\ \mathrm{H} & 0.40689375 & -3.29886543 & -2.49261105 \\ \mathrm{H} & -0.66983320 & -1.89701062 & -2.53140333 \\ \mathrm{H} & 1.07730092 & -1.66637707 & -2.57646002 \\ \mathrm{O} & 1.25823794 & 2.04970860 & -0.26325550 \\ \mathrm{C} & 1.72027994 & 3.34296535 & -0.47943244 \\ \mathrm{H} & 2.24125715 & 3.74179750 & 0.39424822 \\ \mathrm{H} & 2.38460274 & 3.39569839 & -1.34474222 \\ \mathrm{H} & 0.84428884 & 3.95601143 & -0.67351584 \\ \mathrm{H} & -4.24463859 & -0.33849827 & -1.02500139 \\ \mathrm{H} & -2.40311818 & -2.93880500 & -0.24626651 \\ \mathrm{C} & -2.56788533 & -2.43766584 & 1.84261247 \\ \mathrm{H} & -2.96286603 & -3.41616026 & 2.12284260 \\ \mathrm{H} & -1.50009636 & -2.43262414 & 2.06540676 \\ \mathrm{H} & -3.04327339 & -1.68950873 & 2.48012175 \\ \mathrm{H} & -3.91712835 & -2.21569429 & 0.19260347\end{array}$

motor 2c - anti-(M)-stable- $Z$

\begin{tabular}{lrrc}
$\mathrm{C}$ & 2.25464491 & -1.32468516 & -0.09745729 \\
$\mathrm{C}$ & 3.28013786 & -0.82313890 & 0.62307161 \\
$\mathrm{C}$ & 1.02205000 & -0.47205057 & -0.25710446 \\
$\mathrm{~S}$ & 3.31962466 & 0.82291376 & 1.25951107 \\
$\mathrm{C}$ & 2.33075183 & 1.58887270 & 0.01175807 \\
$\mathrm{C}$ & 1.35223678 & 0.93861699 & -0.64585952 \\
$\mathrm{C}$ & -0.23536481 & -0.92350438 & 0.00809750 \\
$\mathrm{C}$ & -1.54531119 & -0.19913732 & 0.03414294 \\
$\mathrm{C}$ & -0.60575376 & -2.38699952 & 0.28110114 \\
$\mathrm{C}$ & -2.58066398 & -1.12040567 & -0.18572481 \\
$\mathrm{H}$ & 0.06609426 & -3.06907138 & -0.22417555 \\
$\mathrm{C}$ & -2.01908520 & -2.51275602 & -0.32420423 \\
$\mathrm{H}$ & -2.63002642 & -3.26127095 & 0.18316983 \\
$\mathrm{C}$ & -1.88106613 & 1.13302325 & 0.35518844 \\
$\mathrm{C}$ & -3.22528661 & 1.52318446 & 0.33057785 \\
$\mathrm{C}$ & -4.23559112 & 0.59833906 & 0.03679315 \\
$\mathrm{C}$ & -3.92273389 & -0.73718913 & -0.20723508 \\
$\mathrm{H}$ & -1.96195832 & -2.80127679 & -1.37792415 \\
$\mathrm{H}$ & -5.26641276 & 0.92784565 & 0.03102765 \\
$\mathrm{H}$ & -4.70363117 & -1.46421930 & -0.38854470 \\
$\mathrm{H}$ & -3.50060383 & 2.54108983 & 0.55962781 \\
$\mathrm{O}$ & -0.88311103 & 1.96439003 & 0.69956536 \\
$\mathrm{C}$ & -1.13330617 & 3.25905436 & 1.13997807 \\
$\mathrm{H}$ & -1.59200784 & 3.87337339 & 0.36154583 \\
$\mathrm{H}$ & -1.77380587 & 3.26858368 & 2.02459783 \\
$\mathrm{H}$ & -0.16996385 & 3.68852457 & 1.40150585 \\
$\mathrm{H}$ & 4.18767992 & -1.38951372 & 0.78827309 \\
$\mathrm{C}$ & 0.70062659 & 1.54894739 & -1.86999066 \\
$\mathrm{H}$ & -0.37428011 & 1.38169579 & -1.84598679 \\
$\mathrm{C}$ & 2.42318724 & -2.66986698 & -0.78038849 \\
$\mathrm{H}$ & 1.87165589 & -2.70584562 & -1.72004708 \\
$\mathrm{H}$ & 3.47412818 & -2.84653590 & -1.00721570 \\
$\mathrm{H}$ & 2.08146128 & -3.49909275 & -0.16034611 \\
$\mathrm{C}$ & -0.58400516 & -2.70712348 & 1.77953447 \\
$\mathrm{H}$ & 0.41244564 & -2.55242859 & 2.19573401 \\
$\mathrm{H}$ & -1.27753435 & -2.07033645 & 2.33214401 \\
$\mathrm{H}$ & -0.86845355 & -3.74726939 & 1.95546435 \\
$\mathrm{H}$ & 0.84717876 & 2.63058582 & -1.84532302 \\
& & & \\
\hline
\end{tabular}




$\begin{array}{rrrr}\text { C } & 1.25995061 & 0.99229178 & -3.18090988 \\ \mathrm{H} & 0.76089339 & 1.44929628 & -4.03823062 \\ \mathrm{H} & 2.33017906 & 1.18821574 & -3.26932326 \\ \mathrm{H} & 1.11113070 & -0.08720390 & -3.24983686 \\ \mathrm{H} & 2.60863258 & 2.60702836 & -0.22811868\end{array}$

motor $2 \mathrm{c}$ - anti-(M)-stable- $E^{*}$

$\begin{array}{lrcc}\mathrm{C} & -2.25401028 & 1.23215768 & 2.24452200 \\ \mathrm{C} & -0.81509815 & -0.10336822 & 0.69701851 \\ \mathrm{~S} & -3.58724999 & 1.20920678 & 1.24289031 \\ \mathrm{C} & -3.12291643 & 0.36662268 & -0.11983495 \\ \mathrm{C} & -1.81892970 & -0.24741092 & -0.29335738 \\ \mathrm{C} & 0.48540547 & -0.82528937 & 0.50997136 \\ \mathrm{C} & 1.64887649 & -0.36620909 & -0.15495952 \\ \mathrm{C} & 0.81196945 & -2.11228513 & 1.26516210 \\ \mathrm{C} & 2.70813422 & -1.30420788 & -0.01100661 \\ \mathrm{H} & 0.89689593 & -1.86067330 & 2.32773559 \\ \mathrm{C} & 2.21335437 & -2.51547613 & 0.74264212 \\ \mathrm{H} & 2.14040580 & -3.37142793 & 0.06528756 \\ \mathrm{C} & 1.88225318 & 0.83291218 & -0.89263739 \\ \mathrm{C} & 3.14235154 & 1.05782629 & -1.43766184 \\ \mathrm{C} & 4.17922737 & 0.11606404 & -1.27212514 \\ \mathrm{C} & 3.96283096 & -1.07205001 & -0.56068314 \\ \mathrm{H} & 2.88654196 & -2.80985635 & 1.54976943 \\ \mathrm{H} & 5.14876135 & 0.32121503 & -1.70582181 \\ \mathrm{H} & 4.76188704 & -1.79318097 & -0.44516228 \\ \mathrm{H} & 3.34492482 & 1.95994992 & -1.99495842 \\ \mathrm{C} & -0.97462120 & 0.63915980 & 1.89296365 \\ \mathrm{C} & -1.58966076 & -0.92913349 & -1.62753192 \\ \mathrm{H} & -1.71250650 & -0.18408873 & -2.42180174 \\ \mathrm{H} & -0.55814718 & -1.26592603 & -1.69666704 \\ \mathrm{C} & 0.12953560 & 0.83819725 & 2.90342291 \\ \mathrm{H} & 1.11385816 & 0.65699926 & 2.47879061 \\ \mathrm{H} & 0.12024163 & 1.86568611 & 3.27463645 \\ \mathrm{H} & 0.01477019 & 0.18318148 & 3.77400225 \\ \mathrm{H} & -2.40564223 & 1.72570977 & 3.19254110 \\ \mathrm{C} & -0.22593279 & -3.22645282 & 1.14785332 \\ \mathrm{H} & 0.08197260 & -4.09454400 & 1.73539267 \\ \mathrm{H} & -1.20048995 & -2.90387636 & 1.51609148 \\ \mathrm{H} & -0.34426814 & -3.54966002 & 0.11210362 \\ \mathrm{O} & 0.83938155 & 1.67666936 & -1.00781683 \\ \mathrm{C} & 0.96375693 & 2.87963214 & -1.69385763 \\ \mathrm{H} & 1.71602050 & 3.52890711 & -1.23973279 \\ \mathrm{H} & 1.21823741 & 2.72236159 & -2.74472625 \\ \mathrm{H} & -0.00322191 & 3.37222563 & -1.63783494 \\ \mathrm{H} & -3.88747383 & 0.29241725 & -0.87752020 \\ \mathrm{C} & -2.51842639 & -2.11110337 & -1.91885046 \\ \mathrm{H} & -3.56823349 & -1.81257032 & -1.93755410 \\ \mathrm{H} & -2.28736071 & -2.54767367 & -2.89317381 \\ \mathrm{H} & -2.41486689 & -2.89384207 & -1.16623865\end{array}$

motor $2 \mathrm{c}$ - anti-(M)-stable- $Z^{*}$

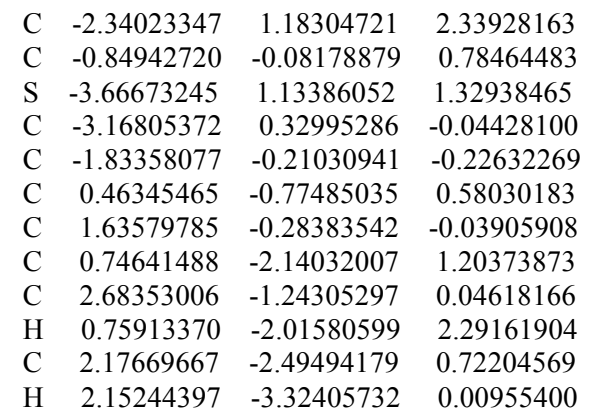




$\begin{array}{lrrl}\mathrm{C} & 1.88886583 & 0.95793231 & -0.69761675 \\ \mathrm{C} & 3.15253282 & 1.20078760 & -1.22428139 \\ \mathrm{C} & 4.17721589 & 0.23653872 & -1.11751981 \\ \mathrm{C} & 3.94297265 & -0.99131511 & -0.48359096 \\ \mathrm{H} & 2.82130076 & -2.80870301 & 1.54516702 \\ \mathrm{H} & 5.15052752 & 0.45633347 & -1.53518309 \\ \mathrm{H} & 4.73223391 & -1.72883657 & -0.41262022 \\ \mathrm{H} & 3.36873151 & 2.13453920 & -1.72106271 \\ \mathrm{C} & -1.04910982 & 0.59973337 & 2.01031450 \\ \mathrm{C} & -1.59007153 & -0.87978696 & -1.55674090 \\ \mathrm{H} & -1.84629657 & -0.20263679 & -2.37652896 \\ \mathrm{H} & -0.55250424 & -1.17220335 & -1.68964055 \\ \mathrm{C} & 0.05510928 & 0.86318989 & 3.01405015 \\ \mathrm{H} & 0.89328387 & 0.19163756 & 2.84409071 \\ \mathrm{H} & -0.31441296 & 0.63618150 & 4.01961125 \\ \mathrm{H} & -2.51554616 & 1.66174961 & 3.29085416 \\ \mathrm{C} & -0.28583336 & -3.22439526 & 0.89220092 \\ \mathrm{H} & -0.01432707 & -4.15816567 & 1.39004607 \\ \mathrm{H} & -1.27949824 & -2.93909064 & 1.23917050 \\ \mathrm{H} & -0.34472720 & -3.42230950 & -0.17961370 \\ \mathrm{O} & 0.85940861 & 1.82506764 & -0.75414697 \\ \mathrm{C} & 0.97753696 & 3.03196630 & -1.43520379 \\ \mathrm{H} & 1.73529046 & 3.67856712 & -0.98659018 \\ \mathrm{H} & 1.22052815 & 2.87824892 & -2.48920793 \\ \mathrm{H} & 0.01190842 & 3.52544240 & -1.36577213 \\ \mathrm{H} & -3.92561558 & 0.22358667 & -0.80618162 \\ \mathrm{H} & -2.20807377 & -1.77539284 & -1.67516912 \\ \mathrm{C} & 0.57293394 & 2.30477366 & 2.98689290 \\ \mathrm{H} & -0.22186461 & 3.02087598 & 3.20597806 \\ \mathrm{H} & 1.36271752 & 2.44792768 & 3.72821467 \\ \mathrm{H} & 0.97629862 & 2.54813552 & 2.00382949\end{array}$

motor $2 \mathrm{c}$ - ( $(P)$-unstable- $Z$

$\begin{array}{lrrr}\text { C } & -2.50638832 & 0.93049604 & 2.22981408 \\ \text { C } & -0.76617582 & -0.11210308 & 0.76359508 \\ \text { S } & -3.54197731 & 1.56212147 & 0.97973256 \\ \text { C } & -2.73043757 & 0.91167621 & -0.41995513 \\ \text { C } & -1.56851179 & 0.21565207 & -0.44956882 \\ \text { C } & 0.49303000 & -0.70785086 & 0.65966511 \\ \text { C } & 1.54437564 & -0.43147994 & -0.34409963 \\ \text { C } & 1.04600709 & -1.82621511 & 1.57069705 \\ \text { C } & 2.57852508 & -1.37227452 & -0.24953867 \\ \text { H } & 1.43336168 & -1.41525498 & 2.50292538 \\ \text { C } & 2.24707203 & -2.41163078 & 0.78429531 \\ \text { H } & 1.96853273 & -3.35263710 & 0.30191122 \\ \text { C } & 1.73657244 & 0.70613933 & -1.16545773 \\ \text { C } & 2.89901340 & 0.80774602 & -1.93350943 \\ \text { C } & 3.89083305 & -0.18269728 & -1.86662671 \\ \text { C } & 3.74410586 & -1.27332506 & -1.01280709 \\ \text { H } & 3.09603859 & -2.63121928 & 1.43376901 \\ \text { H } & 4.78372553 & -0.07613824 & -2.46861984 \\ \text { H } & 4.52481325 & -2.01821544 & -0.92906311 \\ \text { H } & 3.05639687 & 1.66145217 & -2.57464047 \\ \text { C } & -1.35823533 & 0.21840117 & 2.08954577 \\ \text { C } & -1.28296552 & -0.47706420 & -1.77966919 \\ \text { H } & -1.64566873 & 0.16341974 & -2.58541131 \\ \text { H } & -0.22320719 & -0.60097171 & -1.95541487 \\ \text { C } & -0.55896170 & 0.04568301 & 3.37085164 \\ \text { H } & 0.42740691 & 0.49805447 & 3.26365546 \\ \text { H } & -1.06307977 & 0.55040355 & 4.19267960 \\ \text { H } & -0.42055833 & -0.98932978 & 3.66840758 \\ \text { H } & -2.81962859 & 1.25069041 & 3.21360399 \\ \text { C } & 0.03021916 & -2.92831850 & 1.88755342 \\ \text { H } & 0.48524147 & -3.68932882 & 2.52670729 \\ \text { H } & -0.86135823 & -2.56032259 & 2.38968857\end{array}$




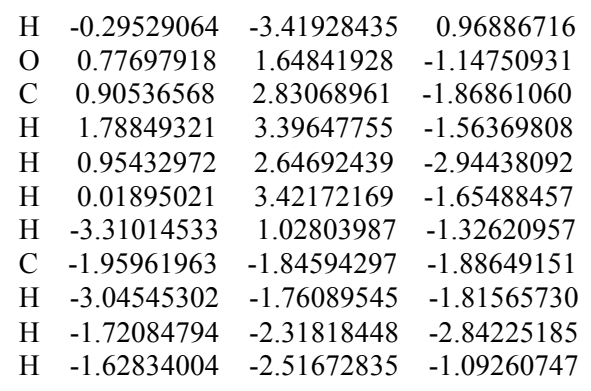

motor 2c - (P)-unstable- $E$

\begin{tabular}{|c|c|c|c|}
\hline $\mathrm{C}$ & -2.72695602 & 0.03004118 & 2.31092160 \\
\hline $\mathrm{C}$ & -0.76917941 & -0.12748407 & 0.73854714 \\
\hline$S$ & -3.95578229 & -0.18499207 & 1.08911167 \\
\hline $\mathrm{C}$ & -2.98575676 & 0.09981831 & -0.34054439 \\
\hline $\mathrm{C}$ & -1.63350944 & 0.14972903 & -0.44391315 \\
\hline $\mathrm{C}$ & 0.52605463 & -0.60918245 & 0.65790414 \\
\hline $\mathrm{C}$ & 1.57363498 & -0.36035478 & -0.36580704 \\
\hline $\mathrm{C}$ & 1.13628996 & -1.70195548 & 1.56258168 \\
\hline $\mathrm{C}$ & 2.43731534 & -1.46107854 & -0.44098152 \\
\hline $\mathrm{H}$ & 57621 & -1.24857643 & 2.26865612 \\
\hline C & 1.98488749 & -2.50630456 & 0.54315512 \\
\hline $\mathrm{H}$ & 1.36110102 & -3.26049606 & 0.05310390 \\
\hline $\mathrm{C}$ & 1.94030817 & 0.84205451 & -1.00377852 \\
\hline C & 3.06783319 & 0.85232774 & -1.83263195 \\
\hline $\mathrm{C}$ & 3.85862568 & -0.29657815 & -1.97740726 \\
\hline $\mathrm{C}$ & 3.56565499 & -1.45770589 & -1.26141715 \\
\hline $\mathrm{H}$ & 2.81503914 & -3.02983852 & 1.01973106 \\
\hline $\mathrm{H}$ & 4.72713412 & -0.25875641 & -2.62194262 \\
\hline $\mathrm{H}$ & 4.21455948 & -2.32175403 & -1.32086175 \\
\hline $\mathrm{H}$ & 3.35887886 & 1.753 & -2.35 \\
\hline $\mathrm{C}$ & -1.39510537 & 0.05809961 & 2.08694343 \\
\hline $\mathrm{C}$ & -1.08731039 & 0.23363100 & -1.85452399 \\
\hline $\mathrm{H}$ & -1.90913765 & 0.23998665 & -2.56867558 \\
\hline $\mathrm{H}$ & -0.49882750 & 1.12992824 & -2.01083424 \\
\hline $\mathrm{C}$ & -0.51617301 & 0.57142426 & 3.22143164 \\
\hline $\mathrm{H}$ & 0.40890432 & 0.00695973 & 3.29294028 \\
\hline $\mathrm{H}$ & -1.03894190 & 0.41908304 & 4.16793644 \\
\hline $\mathrm{H}$ & -3.12477747 & 0.24220846 & 3.29472215 \\
\hline $\mathrm{C}$ & 0.18329146 & -2.62235108 & 2.32107369 \\
\hline $\mathrm{H}$ & 0.74718874 & -3.45795917 & 2.74247967 \\
\hline $\mathrm{H}$ & -0.32960574 & -2.13107804 & 3.14367622 \\
\hline $\mathrm{H}$ & -0.57594361 & -3.03330423 & 1.65345258 \\
\hline $\mathrm{O}$ & 1.20158907 & 1.94173425 & -0.74117138 \\
\hline $\mathrm{C}$ & 1.58381118 & 3.19313301 & -1.21619261 \\
\hline $\mathrm{H}$ & 2.58474985 & 3.46590262 & -0.87492390 \\
\hline $\mathrm{H}$ & 1.55492604 & 3.24043424 & -2.30726017 \\
\hline $\mathrm{H}$ & 0.86982863 & 3.90908392 & -0.81800613 \\
\hline $\mathrm{H}$ & -3.59451158 & 0.16276581 & -1.23173371 \\
\hline $\mathrm{H}$ & -0.46048954 & -0.62768963 & -2.08305784 \\
\hline $\mathrm{C}$ & -0.17318153 & 2.05524998 & 3.06708511 \\
\hline $\mathrm{H}$ & -1.07552039 & 2.66952114 & 3.07854782 \\
\hline $\mathrm{H}$ & 0.47066810 & 2.38711096 & 3.88487177 \\
\hline $\mathrm{H}$ & 0.34881874 & 2.24210701 & 2.12812886 \\
\hline
\end{tabular}




\title{
$\omega B$ 97X-D geometries of stationary points relevant for the thermal isomerizations of motors $2 \mathrm{a}-\mathbf{2 c}$
}

\author{
motor 2a - (P)-unstable- $Z$ \\ $\begin{array}{llll}\text { C } & -2.415223 & 0.663342 & -0.890114\end{array}$ \\ $\begin{array}{llll}\text { C } & -3.568135 & -0.037296 & -0.879304\end{array}$ \\ $\begin{array}{llll}\text { C } & -1.231839 & 0.184888 & -0.122409\end{array}$ \\ C $\quad-2.630546-1.652283 \quad 0.747183$ \\ $\begin{array}{llll}\text { C } & -1.440782 & -1.017989 & 0.731445\end{array}$ \\ $\begin{array}{llll}\text { C } & -0.019042 & 0.834968 & -0.184747\end{array}$ \\ $\begin{array}{llll}\text { C } & 1.321391 & 0.233846 & -0.145058\end{array}$ \\ $\begin{array}{lllll}\text { C } & 0.183994 & 2.360241 & -0.221894\end{array}$ \\ $\begin{array}{llll}\mathrm{C} & 2.303986 & 1.217030 & 0.046084\end{array}$ \\ $\begin{array}{llll}\mathrm{H} & 0.079429 & 2.750451 & -1.247181\end{array}$ \\ $\begin{array}{llll}\text { C } & 1.658891 & 2.560182 & 0.217590\end{array}$ \\ $\begin{array}{llll}\mathrm{H} & 1.699744 & 2.868991 & 1.276749\end{array}$ \\ $\begin{array}{llll}\text { C } & 1.728193 & -1.072506 & -0.507628\end{array}$ \\ $\begin{array}{llll}\text { C } & 3.094687 & -1.382311 & -0.520036\end{array}$ \\ $\begin{array}{lllll}\text { C } & 4.046596 & -0.397650 & -0.242952\end{array}$ \\ $\begin{array}{llll}\text { C } & 3.663132 & 0.916463 & 0.021193\end{array}$ \\ $\begin{array}{llll}\mathrm{H} & 2.174535 & 3.342907 & -0.358586\end{array}$ \\ $\mathrm{H} \quad 5.106303 \quad-0.664069-0.266496$ \\ $\mathrm{H} \quad 4.411131 \quad 1.695852 \quad 0.184191$ \\ $\begin{array}{llll}\mathrm{H} & 3.428454 & -2.389840 & -0.769251\end{array}$ \\ $\begin{array}{llll}\text { C } & -0.460287 & -1.373275 & 1.837213\end{array}$ \\ $\mathrm{H} \quad 0.555843 \quad-1.530806 \quad 1.461999$ \\ $\mathrm{H} \quad-0.774712 \quad-2.338184 \quad 2.264305$ \\ $\begin{array}{llll}\text { C } & -2.276175 & 1.760270 & -1.921859\end{array}$ \\ $\begin{array}{llll}\mathrm{H} & -2.147161 & 2.763078 & -1.494071\end{array}$ \\ $\begin{array}{llll}\text { C } & -0.759924 & 3.124218 & 0.708706\end{array}$ \\ $\begin{array}{llll}\mathrm{H} & -0.568837 & 4.207485 & 0.646553\end{array}$ \\ $\mathrm{H} \quad-1.821091 \quad 2.957014 \quad 0.482080$ \\ $\begin{array}{llll}\mathrm{H} & -0.598183 & 2.813759 & 1.753222\end{array}$ \\ $\mathrm{H} \quad-2.782984 \quad-2.459535 \quad 1.471535$ \\ $\begin{array}{lllll}\mathrm{O} & 0.766717 & -1.945517 & -0.850530\end{array}$ \\ $\begin{array}{llll}\text { C } & 1.102493 & -3.273649 & -1.165951\end{array}$ \\ $\begin{array}{llll}\mathrm{H} & 1.750171 & -3.333117 & -2.057163\end{array}$ \\ $\mathrm{H} \quad 1.600203 \quad-3.779296 \quad-0.321205$ \\ $\begin{array}{lllll}\mathrm{H} & 0.157378 & -3.788860 & -1.378732\end{array}$ \\ $\begin{array}{llll}\text { C } & -3.806258 & -1.272369 & -0.083272\end{array}$ \\ $\begin{array}{lllll}\mathrm{H} & -4.090444 & -2.096932 & -0.766427\end{array}$ \\ $\mathrm{H} \quad \begin{array}{llll}-4.692485 & -1.149499 & 0.568588\end{array}$ \\ $\begin{array}{llll}\mathrm{H} & -4.372119 & 0.268924 & -1.556513\end{array}$ \\ $\mathrm{H} \quad-3.170621 \quad 1.792315 \quad-2.559832$ \\ $\mathrm{H} \quad-1.407659 \quad 1.568001 \quad-2.571041$ \\ $\begin{array}{llll}\text { C } & -0.409955 & -0.332571 & 2.957360\end{array}$ \\ $\begin{array}{llll}\mathrm{H} & -0.050437 & 0.640698 & 2.590659\end{array}$ \\ $\begin{array}{llll}\mathrm{H} & -1.404647 & -0.177244 & 3.404838\end{array}$ \\ H $\quad 0.273260 \quad-0.659265 \quad 3.756706$
}

motor 2a - syn-(M)-stable- $Z$

$\begin{array}{lrrc}\mathrm{C} & 2.590803 & 1.221294 & -1.584383 \\ \mathrm{C} & 1.354192 & -0.014998 & 0.086240 \\ \mathrm{C} & 3.708705 & -0.558943 & -0.296130 \\ \mathrm{C} & 2.658477 & -0.607979 & 0.537787 \\ \mathrm{C} & 0.200655 & -0.720021 & 0.241687 \\ \mathrm{C} & -1.197188 & -0.418152 & -0.175830 \\ \mathrm{C} & 0.180574 & -2.239484 & 0.450629 \\ \mathrm{C} & -1.709753 & -1.548745 & -0.831894 \\ \mathrm{H} & 1.185917 & -2.668916 & 0.351101 \\ \mathrm{C} & -0.701987 & -2.667352 & -0.751410 \\ \mathrm{H} & -1.176912 & -3.649906 & -0.608852\end{array}$




$\begin{array}{rrrr}\mathrm{C} & -2.072830 & 0.632468 & 0.149917 \\ \mathrm{C} & -3.371472 & 0.622555 & -0.384873 \\ \mathrm{C} & -3.814229 & -0.460259 & -1.146497 \\ \mathrm{C} & -3.000959 & -1.575534 & -1.349586 \\ \mathrm{H} & -0.091955 & -2.717560 & -1.669475 \\ \mathrm{H} & -4.832105 & -0.445496 & -1.544125 \\ \mathrm{H} & -3.379157 & -2.457815 & -1.870968 \\ \mathrm{H} & -4.058144 & 1.444289 & -0.180395 \\ \mathrm{C} & 1.538196 & 1.222828 & -0.745907 \\ \mathrm{C} & 2.787873 & -1.148189 & 1.938591 \\ \mathrm{H} & 2.241247 & -2.089271 & 2.090296 \\ \mathrm{C} & 0.659943 & 2.445849 & -0.666636 \\ \mathrm{H} & 0.919614 & 3.102990 & -1.511138 \\ \mathrm{H} & -0.396743 & 2.184156 & -0.791454 \\ \mathrm{H} & 2.735966 & 2.071785 & -2.258788 \\ \mathrm{C} & -0.440917 & -2.682187 & 1.776612 \\ \mathrm{H} & -0.452450 & -3.780754 & 1.851737 \\ \mathrm{H} & 0.119219 & -2.287798 & 2.636928 \\ \mathrm{H} & -1.480041 & -2.327253 & 1.867653 \\ \mathrm{O} & -1.634781 & 1.565302 & 1.015174 \\ \mathrm{C} & -2.468376 & 2.646317 & 1.353049 \\ \mathrm{H} & -2.718353 & 3.262764 & 0.472731 \\ \mathrm{H} & -3.401628 & 2.305552 & 1.832339 \\ \mathrm{H} & -1.909034 & 3.260505 & 2.069105 \\ \mathrm{H} & 4.673730 & -0.976144 & 0.009613 \\ \mathrm{H} & 3.843367 & -1.337110 & 2.183070 \\ \mathrm{H} & 2.389466 & -0.425471 & 2.668845 \\ \mathrm{C} & 0.855897 & 3.209157 & 0.642494 \\ \mathrm{H} & 1.895953 & 3.560477 & 0.736006 \\ \mathrm{H} & 0.196480 & 4.089861 & 0.689132 \\ \mathrm{H} & 0.630471 & 2.571989 & 1.510028 \\ \mathrm{C} & 3.610032 & 0.120332 & -1.628778 \\ \mathrm{H} & 4.590192 & 0.524756 & -1.926572 \\ \mathrm{H} & 3.347327 & -0.611790 & -2.418681\end{array}$

motor 2a - anti-(M)-stable- $Z$

$\begin{array}{lrrr}\mathrm{C} & 2.418866 & -1.859601 & -0.175618 \\ \mathrm{C} & 1.266128 & 0.277961 & 0.110983 \\ \mathrm{C} & 3.523331 & 0.256191 & -0.786156 \\ \mathrm{C} & 2.571060 & 0.969849 & -0.157996 \\ \mathrm{C} & 0.051194 & 0.887877 & 0.026029 \\ \mathrm{C} & -1.303638 & 0.279302 & 0.001065 \\ \mathrm{C} & -0.211750 & 2.395152 & -0.045292 \\ \mathrm{C} & -2.256048 & 1.232114 & 0.393392 \\ \mathrm{H} & 0.546041 & 2.960009 & 0.503396 \\ \mathrm{C} & -1.578488 & 2.548136 & 0.657438 \\ \mathrm{H} & -2.160127 & 3.402845 & 0.279804 \\ \mathrm{C} & -1.750857 & -0.972645 & -0.472105 \\ \mathrm{C} & -3.116776 & -1.282322 & -0.398211 \\ \mathrm{C} & -4.031884 & -0.339020 & 0.074271 \\ \mathrm{C} & -3.615185 & 0.936023 & 0.454024 \\ \mathrm{H} & -1.439546 & 2.700081 & 1.741471 \\ \mathrm{H} & -5.092023 & -0.602147 & 0.112589 \\ \mathrm{H} & -4.337867 & 1.689886 & 0.774296 \\ \mathrm{H} & -3.478920 & -2.253151 & -0.737960 \\ \mathrm{C} & 1.437309 & -1.174371 & 0.430622 \\ \mathrm{H} & 4.475069 & 0.736306 & -1.036356 \\ \mathrm{C} & 2.862748 & 2.392624 & 0.252709 \\ \mathrm{H} & 2.384458 & 3.136628 & -0.401156 \\ \mathrm{H} & 2.529170 & 2.590042 & 1.282999 \\ \mathrm{H} & 3.946363 & 2.574322 & 0.211008 \\ \mathrm{C} & 0.657318 & -1.786055 & 1.572564 \\ \mathrm{H} & -0.419944 & -1.599273 & 1.475371 \\ \mathrm{C} & -0.253012 & 2.895306 & -1.491956 \\ \mathrm{H} & -1.046915 & 2.387792 & -2.063736\end{array}$




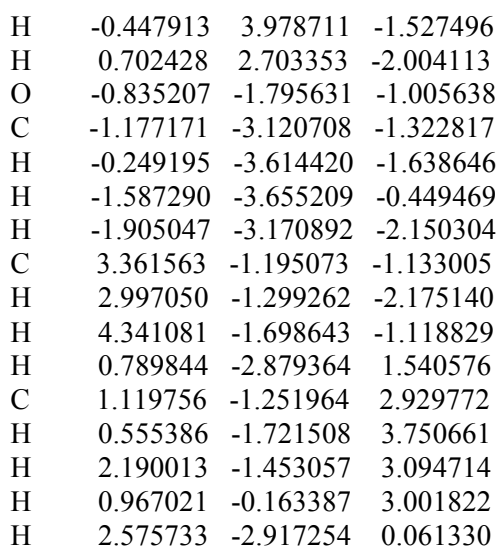

\section{motor 2a-TS1}

$\begin{array}{llll}\text { C } & -2.526415 & -0.961157 & -0.158341\end{array}$

$\begin{array}{llll}\text { C } & -3.156165 & -1.079570 & 1.020276\end{array}$

$\begin{array}{llll}\text { C } & -1.266110 & -0.136329 & -0.115040\end{array}$

$\begin{array}{llll}\text { C } & -2.128387 & 1.030131 & 1.780374\end{array}$

$\begin{array}{llll}\text { C } & -1.515801 & 1.165063 & 0.592736\end{array}$

$\begin{array}{llll}\text { C } & -0.066915 & -0.724218 & -0.353767\end{array}$

$\begin{array}{llll}\text { C } & 1.326324 & -0.307954 & 0.001552\end{array}$

$\begin{array}{llll}\text { C } & 0.060156 & -2.219520 & -0.659167\end{array}$

$\begin{array}{llll}\text { C } & 1.998777 & -1.442270 & 0.492024\end{array}$

$\mathrm{H} \quad-0.883556 \quad-2.743986 \quad-0.467033$

$\begin{array}{llll}\text { C } & 1.127790 & -2.662614 & 0.360362\end{array}$

$\begin{array}{llll}\mathrm{H} & 1.693539 & -3.550443 & 0.039254\end{array}$

$\begin{array}{llll}\text { C } & 2.046038 & 0.898472 & -0.103617\end{array}$

$\begin{array}{llll}\text { C } & 3.348795 & 0.968471 & 0.415951\end{array}$

$\begin{array}{llll}\text { C } & 3.957120 & -0.156936 & 0.970976\end{array}$

$\begin{array}{llll}\text { C } & 3.296459 & -1.382800 & 0.991329\end{array}$

$\begin{array}{llll}\mathrm{H} & 0.654674 & -2.900907 & 1.328176\end{array}$

$\mathrm{H} \quad 4.973821 \quad-0.071687 \quad 1.362626$

$\begin{array}{llll}\mathrm{H} & 3.784630 & -2.279614 & 1.379494\end{array}$

$\begin{array}{llll}\mathrm{H} & 3.908208 & 1.902446 & 0.365882\end{array}$

$\begin{array}{llll}\text { C } & -1.371775 & 2.502928 & -0.088608\end{array}$

$\begin{array}{llll}\mathrm{H} & -0.469158 & 2.516099 & -0.703117\end{array}$

$\mathrm{H} \quad-1.267355 \quad 3.290862 \quad 0.675291$

$\begin{array}{llll}\text { C } & -3.048410 & -1.517122 & -1.452240\end{array}$

$\mathrm{H} \quad \begin{array}{llll}\mathrm{C} & -3.271211 & -0.695147 & -2.152938\end{array}$

$\begin{array}{llll}\text { C } & 0.502214 & -2.488350 & -2.099474\end{array}$

$\mathrm{H} \quad 0.604045-3.569832 \quad-2.280191$

$\mathrm{H} \quad-0.225546 \quad-2.088286 \quad-2.821829$

$\mathrm{H} \quad 1.475636-2.017492 \quad-2.311630$

$\begin{array}{llll}\mathrm{H} & -2.379691 & 1.913055 & 2.378385\end{array}$

$\begin{array}{llll}\text { O } & 1.476016 & 1.925230 & -0.758216\end{array}$

$\begin{array}{llll}\text { C } & 2.142767 & 3.161986 & -0.847516\end{array}$

$\begin{array}{llll}\mathrm{H} & 2.320185 & 3.603406 & 0.147416\end{array}$

$\begin{array}{llll}\mathrm{H} & 3.101774 & 3.069961 & -1.383645\end{array}$

$\begin{array}{llll}\mathrm{H} & 1.483914 & 3.828591 & -1.418643\end{array}$

$\begin{array}{llll}\text { C } & -2.638623 & -0.320757 & 2.222270\end{array}$

$\mathrm{H} \quad-1.829372 \quad-0.900942 \quad 2.709130$

$\begin{array}{llll}\mathrm{H} & -3.425042 & -0.207281 & 2.982379\end{array}$

$\mathrm{H} \quad-4.073185 \quad-1.670120 \quad 1.115248$

$\mathrm{H} \quad-3.975542 \quad-2.087013 \quad-1.291972$

$\mathrm{H} \quad-2.325784 \quad-2.172340 \quad-1.959882$

C $\quad-2.585735 \quad 2.803601 \quad-0.972130$

$\mathrm{H} \quad-2.710735 \quad 2.033120 \quad-1.750230$

$\mathrm{H} \quad \begin{array}{lllll}-3.515556 & 2.836147 & -0.382374\end{array}$

$\mathrm{H} \quad-2.469454 \quad 3.773222 \quad-1.481790$

motor $2 \mathrm{a}-\mathrm{TS} 2$ 


\begin{tabular}{|c|c|c|c|}
\hline & 0.579613 & 2.042617 & -1.156361 \\
\hline & 1.639631 & 1.069992 & -0.653365 \\
\hline $\mathrm{C}$ & 2.909409 & 1.401671 & -0.965118 \\
\hline $\mathrm{C}$ & 1.355363 & -0.263782 & -0.031497 \\
\hline $\mathrm{C}$ & 3.812342 & -0.572446 & 0.196233 \\
\hline $\mathrm{C}$ & 2.564829 & -0.986320 & 0.484489 \\
\hline C & 2.412007 & -2.086870 & 1.508364 \\
\hline C & 0.127195 & -0.874735 & -0.016926 \\
\hline $\mathrm{C}$ & -1.234063 & -0.313183 & -0.185656 \\
\hline $\mathrm{C}$ & -0.113667 & -2.387849 & -0.170380 \\
\hline C & -2.001005 & -1.182257 & -0.972558 \\
\hline $\mathrm{H}$ & 0.799252 & -2.907293 & -0.488152 \\
\hline $\mathrm{C}$ & -1.153408 & -2.376379 & -1.326605 \\
\hline $\mathrm{H}$ & -1.731507 & -3.310339 & -1.390661 \\
\hline C & -1.861397 & 0.753202 & 0.483177 \\
\hline $\mathrm{C}$ & -3.217512 & 1.004445 & 0.227453 \\
\hline $\mathrm{C}$ & -3.938653 & 0.175905 & -0.637025 \\
\hline $\mathrm{C}$ & -3.346542 & -0.939194 & -1.231642 \\
\hline $\mathrm{H}$ & -0.644655 & -2.224935 & -2.293822 \\
\hline $\mathrm{H}$ & -4.994976 & 0.392456 & -0.815227 \\
\hline $\mathrm{H}$ & -3.932124 & -1.617489 & -1.856351 \\
\hline $\mathrm{C}$ & -0.723362 & -3.090240 & 1.047701 \\
\hline $\mathrm{H}$ & -0.068626 & -3.050082 & 1.927174 \\
\hline $\mathrm{H}$ & -1.684459 & -2.629721 & 1.325968 \\
\hline $\mathrm{H}$ & -0.915106 & -4.150069 & 0.818084 \\
\hline $\mathrm{H}$ & -3.727435 & 1.836840 & 0.712699 \\
\hline $\mathrm{H}$ & 4.659754 & -1.088840 & 0.658926 \\
\hline $\mathrm{H}$ & 3.074687 & 2.326160 & -1.529446 \\
\hline $\mathrm{H}$ & -0.420330 & 1.609619 & -1.112066 \\
\hline $\mathrm{H}$ & 3.372479 & -2.270000 & 2.010441 \\
\hline $\mathrm{H}$ & 1.678046 & -1.800527 & 2.276524 \\
\hline $\mathrm{H}$ & 2.082888 & -3.043715 & 1.081224 \\
\hline $\mathrm{O}$ & -1.114676 & 1.456722 & 1.352978 \\
\hline $\mathrm{C}$ & -1.716538 & 2.482236 & 2.105741 \\
\hline $\mathrm{H}$ & -0.940651 & 2.879000 & 2.772393 \\
\hline$\pi$ & -2.089075 & 3.299162 & 1.465354 \\
\hline $\mathrm{H}$ & -2.547886 & 2.098678 & 2.721313 \\
\hline $\mathrm{H}$ & 0.767782 & 2.219875 & -2.227753 \\
\hline C & 0.595497 & 3.388878 & -0.432788 \\
\hline $\mathrm{H}$ & 1.540088 & 3.926839 & -0.611069 \\
\hline $\mathrm{H}$ & -0.225206 & 4.034255 & -0.784573 \\
\hline $\mathrm{H}$ & 0.486698 & 3.258356 & 0.652469 \\
\hline C & 4.124270 & 0.597061 & -0.665095 \\
\hline $\mathrm{H}$ & 4.889602 & 1.227153 & -0.175476 \\
\hline$H$ & 4.604111 & 0.274328 & -1.610642 \\
\hline
\end{tabular}

motor $2 \mathrm{a}-\mathrm{TS} 3$

$\begin{array}{lrrc}\mathrm{C} & 2.460154 & -0.847406 & -0.324154 \\ \mathrm{C} & 3.123823 & -0.971557 & 0.833533 \\ \mathrm{C} & 1.137169 & -0.139681 & -0.203111 \\ \mathrm{C} & 1.984612 & 1.039276 & 1.672764 \\ \mathrm{C} & 1.371770 & 1.179772 & 0.485353 \\ \mathrm{C} & -0.039643 & -0.796515 & -0.379778 \\ \mathrm{C} & -1.446498 & -0.342783 & -0.116304 \\ \mathrm{C} & -0.182911 & -2.302454 & -0.725419 \\ \mathrm{C} & -2.257940 & -1.460133 & 0.141379 \\ \mathrm{H} & -0.390529 & -2.356796 & -1.810629 \\ \mathrm{C} & -1.469125 & -2.719917 & 0.010730 \\ \mathrm{H} & -1.214648 & -3.111226 & 1.012279 \\ \mathrm{C} & -2.075561 & 0.923583 & -0.124091 \\ \mathrm{C} & -3.431697 & 1.030041 & 0.222453 \\ \mathrm{C} & -4.185883 & -0.101810 & 0.528683 \\ \mathrm{C} & -3.607110 & -1.364461 & 0.472840 \\ \mathrm{H} & -2.019003 & -3.515361 & -0.514502 \\ \mathrm{H} & -5.240906 & 0.014769 & 0.788436\end{array}$




$\begin{array}{lrrr}\mathrm{H} & -4.191255 & -2.264971 & 0.675749 \\ \mathrm{H} & -3.915597 & 2.006161 & 0.235791 \\ \mathrm{O} & -1.357609 & 1.987067 & -0.510725 \\ \mathrm{C} & -1.955359 & 3.257744 & -0.574318 \\ \mathrm{H} & -2.811516 & 3.270716 & -1.269333 \\ \mathrm{H} & -2.285684 & 3.606198 & 0.418589 \\ \mathrm{H} & -1.188686 & 3.945935 & -0.952156 \\ \mathrm{H} & 4.093952 & -1.475373 & 0.892985 \\ \mathrm{C} & 1.300416 & 2.520034 & -0.221356 \\ \mathrm{H} & 0.714225 & 2.421691 & -1.139922 \\ \mathrm{H} & 0.778992 & 3.256110 & 0.411038 \\ \mathrm{C} & 2.989496 & -1.214492 & -1.680838 \\ \mathrm{H} & 3.357587 & -0.306278 & -2.188064 \\ \mathrm{H} & 3.826286 & -1.925977 & -1.616350 \\ \mathrm{H} & 2.211464 & -1.637627 & -2.333212 \\ \mathrm{C} & 0.909047 & -3.317760 & -0.391152 \\ \mathrm{H} & 1.800711 & -3.253700 & -1.021629 \\ \mathrm{H} & 1.221290 & -3.238437 & 0.660349 \\ \mathrm{H} & 0.488379 & -4.326021 & -0.536753 \\ \mathrm{C} & 2.541089 & -0.311645 & 2.068880 \\ \mathrm{H} & 1.746806 & -0.957172 & 2.493872 \\ \mathrm{H} & 3.300407 & -0.203231 & 2.856248 \\ \mathrm{C} & 2.693272 & 3.051105 & -0.570970 \\ \mathrm{H} & 2.620094 & 4.003633 & -1.120244 \\ \mathrm{H} & 3.297419 & 3.227420 & 0.332162 \\ \mathrm{H} & 3.244861 & 2.339756 & -1.206183 \\ \mathrm{H} & 2.261503 & 1.917605 & 2.266533\end{array}$

motor 2 a - (P)-unstable- $E$

$\begin{array}{lrrr}\mathrm{C} & 2.414070 & 0.455763 & 0.405019 \\ \mathrm{C} & 3.570719 & -0.217817 & 0.240200 \\ \mathrm{C} & 1.174534 & 0.024967 & -0.298893 \\ \mathrm{C} & 2.477312 & -1.790584 & -1.332309 \\ \mathrm{C} & 1.282411 & -1.190786 & -1.151595 \\ \mathrm{C} & 0.004235 & 0.742068 & -0.201314 \\ \mathrm{C} & -1.369725 & 0.221522 & -0.155193 \\ \mathrm{C} & -0.120228 & 2.273416 & -0.176637 \\ \mathrm{C} & -2.296008 & 1.247082 & -0.398640 \\ \mathrm{H} & -0.073372 & 2.650010 & 0.861473 \\ \mathrm{C} & -1.561890 & 2.520793 & -0.697751 \\ \mathrm{H} & -1.535871 & 2.699185 & -1.787394 \\ \mathrm{C} & -1.836897 & -1.014608 & 0.346404 \\ \mathrm{C} & -3.218837 & -1.231829 & 0.438228 \\ \mathrm{C} & -4.119482 & -0.218968 & 0.098965 \\ \mathrm{C} & -3.668417 & 1.039168 & -0.300364 \\ \mathrm{H} & -2.036578 & 3.400955 & -0.239717 \\ \mathrm{H} & -5.191282 & -0.414078 & 0.185686 \\ \mathrm{H} & -4.374193 & 1.846860 & -0.507550 \\ \mathrm{H} & -3.603528 & -2.185885 & 0.799269 \\ \mathrm{C} & 0.145510 & -1.612368 & -2.052838 \\ \mathrm{H} & -0.655127 & -2.139134 & -1.520298 \\ \mathrm{H} & -0.308797 & -0.740244 & -2.548063 \\ \mathrm{H} & 0.518790 & -2.287809 & -2.835660 \\ \mathrm{C} & 2.346619 & 1.448674 & 1.553965 \\ \mathrm{H} & 1.952439 & 2.423139 & 1.240938 \\ \mathrm{C} & 0.897763 & 3.013085 & -1.042878 \\ \mathrm{H} & 0.697458 & 4.096314 & -1.027100 \\ \mathrm{H} & 1.936816 & 2.862839 & -0.721465 \\ \mathrm{H} & 0.825549 & 2.671397 & -2.087838 \\ \mathrm{H} & 2.552002 & -2.593827 & -2.072620 \\ \mathrm{O} & -0.916735 & -1.908055 & 0.751847 \\ \mathrm{C} & -1.327190 & -3.152889 & 1.260558 \\ \mathrm{H} & -1.942453 & -3.041422 & 2.169426 \\ \mathrm{H} & -1.889479 & -3.736396 & 0.512046 \\ \mathrm{H} & -0.411141 & -3.698928 & 1.518532\end{array}$




$\begin{array}{llll}\mathrm{C} & 3.746506 & -1.382864 & -0.668552 \\ \mathrm{H} & 4.178816 & -2.228970 & -0.100107 \\ \mathrm{H} & 4.510434 & -1.163789 & -1.440098 \\ \mathrm{H} & 4.430260 & 0.054052 & 0.862233 \\ \mathrm{H} & 3.376995 & 1.642638 & 1.889951 \\ \mathrm{C} & 1.528471 & 0.943671 & 2.744218 \\ \mathrm{H} & 0.477981 & 0.758563 & 2.473650 \\ \mathrm{H} & 1.941715 & 0.000872 & 3.136376 \\ \mathrm{H} & 1.539582 & 1.683116 & 3.560002\end{array}$

motor 2a - syn-(M)-stable- $E$

$\begin{array}{llll}\text { C } & 2.419563 & 1.906553 & -1.486813\end{array}$

$\begin{array}{llll}\text { C } & 1.271658 & 0.209205 & -0.170855\end{array}$

$\begin{array}{llll}\text { C } & 3.693351 & 0.003746 & -0.569085\end{array}$

$\begin{array}{llll}\text { C } & 2.618986 & -0.371029 & 0.144810\end{array}$

$\begin{array}{llll}\text { C } & 0.155007 & -0.568444 & -0.077339\end{array}$

$\begin{array}{llll}\text { C } & -1.282891 & -0.239603 & -0.259543\end{array}$

$\begin{array}{llll}\text { C } & 0.176707 & -2.098103 & -0.202006\end{array}$

$\begin{array}{llll}\text { C } & -1.864161 & -1.221395 & -1.075205\end{array}$

$\mathrm{H} \quad 1.169160-2.458938 \quad-0.502633$

C $\quad-0.834674 \quad-2.284151 \quad-1.366380$

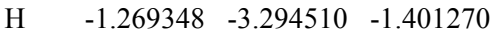

$\begin{array}{llll}\text { C } & -2.111408 & 0.689468 & 0.393783\end{array}$

$\begin{array}{llll}\text { C } & -3.469631 & 0.742885 & 0.043068\end{array}$

$\begin{array}{llll}\text { C } & -4.000937 & -0.171412 & -0.870266\end{array}$

$\begin{array}{llll}\text { C } & -3.213279 & -1.185927 & -1.415268\end{array}$

$\mathrm{H} \quad-0.335340 \quad-2.097616 \quad-2.332399$

$\mathrm{H} \quad \begin{array}{rrrr}-5.063750 & -0.111007 & -1.117807\end{array}$

$\mathrm{H} \quad-3.649483 \quad-1.947000 \quad-2.066315$

$\begin{array}{llll}\mathrm{H} & -4.130498 & 1.476799 & 0.504987\end{array}$

$\begin{array}{llll}\text { C } & 1.329436 & 1.586175 & -0.762572\end{array}$

$\begin{array}{llll}\text { C } & 2.797047 & -1.253587 & 1.364933\end{array}$

$\begin{array}{llll}\mathrm{H} & 2.307753 & -2.228561 & 1.230232\end{array}$

$\begin{array}{llll}\text { C } & 0.206705 & 2.583223 & -0.674297\end{array}$

$\begin{array}{llll}\mathrm{H} & -0.023641 & 2.832742 & 0.368598\end{array}$

$\mathrm{H} \quad 0.485963 \quad 3.506521 \quad-1.201355$

$\begin{array}{llll}\mathrm{H} & -0.718615 & 2.204829 & -1.132004\end{array}$

$\mathrm{H} \quad 2.443176 \quad 2.872521 \quad-2.001520$

$\begin{array}{llll}\text { C } & -0.293777 & -2.851298 & 1.045118\end{array}$

$\mathrm{H} \quad-0.272054 \quad-3.937830 \quad 0.867361$

$\begin{array}{llll}\mathrm{H} & 0.339422 & -2.638705 & 1.918208\end{array}$

$\mathrm{H} \quad-1.326825 \quad-2.575384 \quad 1.310229$

$\begin{array}{llll}\mathrm{O} & -1.561554 & 1.438100 & 1.367205\end{array}$

$\begin{array}{llll}\text { C } & -2.333050 & 2.433462 & 1.994551\end{array}$

$\begin{array}{llll}\mathrm{H} & -2.691701 & 3.186220 & 1.272328\end{array}$

$\begin{array}{llll}\mathrm{H} & -3.197642 & 2.004219 & 2.528423\end{array}$

$\begin{array}{llll}\mathrm{H} & -1.676351 & 2.923824 & 2.723930\end{array}$

$\begin{array}{llll}\mathrm{H} & 4.678861 & -0.401428 & -0.316247\end{array}$

$\begin{array}{llll}\mathrm{H} & 3.871315 & -1.472300 & 1.468272\end{array}$

$\begin{array}{llll}\text { C } & 2.287997 & -0.615809 & 2.657547\end{array}$

$\begin{array}{llll}\mathrm{H} & 2.475804 & -1.277787 & 3.517024\end{array}$

$\begin{array}{llll}\mathrm{H} & 2.793579 & 0.343556 & 2.851553\end{array}$

$\begin{array}{llll}\mathrm{H} & 1.206255 & -0.417719 & 2.614740\end{array}$

$\begin{array}{llll}\text { C } & 3.609959 & 1.016578 & -1.663528\end{array}$

$\begin{array}{llll}\mathrm{H} & 4.533660 & 1.618432 & -1.691245\end{array}$

$\begin{array}{llll}\mathrm{H} & 3.567078 & 0.521097 & -2.654649\end{array}$

motor 2a - anti-(M)-stable- $E$

$\begin{array}{llll}\text { C } & -1.773818 & 2.523813 & 0.197198\end{array}$

$\begin{array}{llll}\text { C } & -1.179822 & 0.155695 & 0.151678\end{array}$

$\begin{array}{llll}\text { C } & -3.301284 & 0.859596 & -0.787720\end{array}$

$\begin{array}{llll}\text { C } & -2.595458 & -0.151740 & -0.249874\end{array}$

$\begin{array}{llll}\text { C } & -0.129967 & -0.691114 & -0.038002\end{array}$

$\begin{array}{llll}\text { C } & 1.327436 & -0.410061 & 0.048795\end{array}$ 


\begin{tabular}{rrrr}
$\mathrm{C}$ & -0.207237 & -2.182447 & -0.377226 \\
$\mathrm{C}$ & 2.026166 & -1.608318 & 0.261351 \\
$\mathrm{H}$ & -1.089751 & -2.649040 & 0.068148 \\
$\mathrm{C}$ & 1.062427 & -2.761760 & 0.284053 \\
$\mathrm{H}$ & 1.453657 & -3.646990 & -0.239851 \\
$\mathrm{C}$ & 2.062965 & 0.771959 & -0.183108 \\
$\mathrm{C}$ & 3.458947 & 0.744964 & -0.046872 \\
$\mathrm{C}$ & 4.119267 & -0.450115 & 0.245131 \\
$\mathrm{C}$ & 3.413101 & -1.644405 & 0.379941 \\
$\mathrm{H}$ & 0.851158 & -3.063204 & 1.324374 \\
$\mathrm{H}$ & 5.208412 & -0.444163 & 0.336271 \\
$\mathrm{H}$ & 3.934352 & -2.587942 & 0.556852 \\
$\mathrm{H}$ & 4.042146 & 1.653578 & -0.198594 \\
$\mathrm{C}$ & -1.030766 & 1.534463 & 0.714873 \\
$\mathrm{C}$ & -3.263677 & -1.490937 & -0.023973 \\
$\mathrm{H}$ & -2.821303 & -2.273883 & -0.657831 \\
$\mathrm{C}$ & -0.193210 & 1.742863 & 1.948504 \\
$\mathrm{H}$ & 0.800882 & 1.282443 & 1.890847 \\
$\mathrm{H}$ & -0.064295 & 2.814726 & 2.159090 \\
$\mathrm{H}$ & -0.696120 & 1.286563 & 2.818280 \\
$\mathrm{H}$ & -1.695607 & 3.538868 & 0.600327 \\
$\mathrm{C}$ & -0.229778 & -2.424652 & -1.888396 \\
$\mathrm{H}$ & -0.272567 & -3.502637 & -2.110361 \\
$\mathrm{H}$ & -1.105149 & -1.945206 & -2.352849 \\
$\mathrm{H}$ & 0.672520 & -2.013380 & -2.369177 \\
$\mathrm{O}$ & 1.380808 & 1.863594 & -0.560916 \\
$\mathrm{C}$ & 2.022069 & 3.112051 & -0.618852 \\
$\mathrm{H}$ & 2.491978 & 3.373849 & 0.344233 \\
$\mathrm{H}$ & 2.786643 & 3.144347 & -1.413386 \\
$\mathrm{H}$ & 1.243618 & 3.851094 & -0.847759 \\
$\mathrm{C}$ & -2.763634 & 2.257499 & -0.898109 \\
$\mathrm{H}$ & -3.588814 & 2.985627 & -0.859360 \\
$\mathrm{H}$ & -2.291188 & 2.404549 & -1.890611 \\
$\mathrm{H}$ & -4.324825 & 0.677431 & -1.131708 \\
$\mathrm{C}$ & -3.246550 & -1.931238 & 1.439425 \\
$\mathrm{H}$ & -3.767463 & -1.197487 & 2.074406 \\
$\mathrm{H}$ & -3.748319 & -2.903303 & 1.563244 \\
$\mathrm{H}$ & -2.220624 & -2.031294 & 1.827615 \\
$\mathrm{H}$ & -4.308860 & -1.399991 & -0.357764 \\
& & & \\
\hline
\end{tabular}

motor 2a - TS4

$\begin{array}{rrrr}\mathrm{C} & -2.528805 & 0.097516 & 0.016446 \\ \mathrm{C} & -3.052619 & 0.420524 & 1.208725 \\ \mathrm{C} & -1.074684 & 0.463742 & -0.137357 \\ \mathrm{C} & -1.364645 & 2.205622 & 1.460876 \\ \mathrm{C} & -0.847342 & 1.893038 & 0.261795 \\ \mathrm{C} & -0.139843 & -0.512402 & -0.252836 \\ \mathrm{C} & 1.330388 & -0.494457 & 0.008677 \\ \mathrm{C} & -0.495403 & -2.002010 & -0.292076 \\ \mathrm{C} & 1.674347 & -1.696149 & 0.651776 \\ \mathrm{H} & -1.534457 & -2.170774 & 0.016985 \\ \mathrm{C} & 0.474943 & -2.599925 & 0.745010 \\ \mathrm{H} & 0.723768 & -3.653933 & 0.548597 \\ \mathrm{C} & 2.352032 & 0.432314 & -0.272745 \\ \mathrm{C} & 3.652881 & 0.182970 & 0.192628 \\ \mathrm{C} & 3.948571 & -0.992831 & 0.883084 \\ \mathrm{C} & 2.965661 & -1.955173 & 1.102602 \\ \mathrm{H} & 0.036117 & -2.546214 & 1.755877 \\ \mathrm{H} & 4.971772 & -1.162926 & 1.227226 \\ \mathrm{H} & 3.200066 & -2.894469 & 1.608711 \\ \mathrm{H} & 4.450824 & 0.899427 & -0.001840 \\ \mathrm{C} & -0.313242 & 2.942973 & -0.685137 \\ \mathrm{H} & 0.036014 & 2.502910 & -1.624169 \\ \mathrm{H} & -1.115091 & 3.661199 & -0.920801 \\ \mathrm{H} & 0.518870 & 3.513010 & -0.246561\end{array}$




$\begin{array}{lrrr}\mathrm{C} & -3.292644 & -0.494874 & -1.141755 \\ \mathrm{H} & -2.746220 & -1.359528 & -1.547105 \\ \mathrm{C} & -0.274703 & -2.613047 & -1.678927 \\ \mathrm{H} & -0.529613 & -3.684469 & -1.676225 \\ \mathrm{H} & -0.892249 & -2.116119 & -2.442227 \\ \mathrm{H} & 0.778178 & -2.517129 & -1.990017 \\ \mathrm{H} & -1.283347 & 3.224239 & 1.856007 \\ \mathrm{O} & 2.039078 & 1.498087 & -1.026139 \\ \mathrm{C} & 3.006264 & 2.484974 & -1.283985 \\ \mathrm{H} & 3.375422 & 2.948946 & -0.353856 \\ \mathrm{H} & 3.861665 & 2.081041 & -1.851045 \\ \mathrm{H} & 2.513771 & 3.253873 & -1.892685 \\ \mathrm{C} & -2.216616 & 1.201692 & 2.199993 \\ \mathrm{H} & -1.579641 & 0.505625 & 2.781334 \\ \mathrm{H} & -2.857796 & 1.707584 & 2.936157 \\ \mathrm{H} & -4.090791 & 0.190662 & 1.463533 \\ \mathrm{H} & -3.276755 & 0.253508 & -1.954561 \\ \mathrm{C} & -4.730177 & -0.898111 & -0.846638 \\ \mathrm{H} & -4.778738 & -1.662577 & -0.055183 \\ \mathrm{H} & -5.337344 & -0.038403 & -0.523218 \\ \mathrm{H} & -5.203239 & -1.318500 & -1.746752\end{array}$

motor 2a - TS5

$\begin{array}{llll}\text { C } & -0.039296 & -2.619167 & -0.549746\end{array}$

$\begin{array}{llll}\text { C } & -1.163754 & -1.639222 & -0.743391\end{array}$

$\begin{array}{llll}\text { C } & -2.184715 & -1.979159 & -1.553813\end{array}$

$\begin{array}{llll}\text { C } & -1.180175 & -0.264266 & -0.146427\end{array}$

$\begin{array}{llll}\text { C } & -3.552962 & -0.080739 & -0.769415\end{array}$

$\begin{array}{llll}\text { C } & -2.556435 & 0.294992 & 0.047974\end{array}$

$\begin{array}{llll}\text { C } & -2.855620 & 1.191223 & 1.238887\end{array}$

$\begin{array}{llll}\text { C } & -0.097171 & 0.549858 & 0.015528\end{array}$

$\begin{array}{llll}\text { C } & 1.355170 & 0.279603 & -0.161676\end{array}$

$\begin{array}{llll}\text { C } & -0.177240 & 2.080049 & -0.097243\end{array}$

$\begin{array}{llll}\mathrm{C} & 1.888601 & 1.283300 & -0.983906\end{array}$

H $\quad-1.187374 \quad 2.407698 \quad-0.376719$

$\begin{array}{llll}\text { C } & 0.813821 & 2.301991 & -1.272770\end{array}$

$\begin{array}{llll}\mathrm{H} & 1.207263 & 3.328754 & -1.317756\end{array}$

$\begin{array}{llll}\text { C } & 2.225967 & -0.619373 & 0.477803\end{array}$

$\begin{array}{llll}\text { C } & 3.582145 & -0.616626 & 0.114220\end{array}$

$\begin{array}{lllll}\text { C } & 4.068505 & 0.320967 & -0.799943\end{array}$

$\begin{array}{llll}\mathrm{C} & 3.234802 & 1.302892 & -1.336158\end{array}$

$\mathrm{H} \quad \begin{array}{llll}\mathrm{H} & 0.314187 & 2.088158 & -2.232942\end{array}$

$\mathrm{H} \quad \begin{array}{lllll} & 5.130784 & 0.304914 & -1.056488\end{array}$

$\begin{array}{llll}\mathrm{H} & 3.633041 & 2.081284 & -1.991074\end{array}$

$\begin{array}{llll}\text { C } & 0.288989 & 2.852254 & 1.139972\end{array}$

$\begin{array}{llll}\mathrm{H} & -0.331078 & 2.638121 & 2.021967\end{array}$

$\begin{array}{llll}\mathrm{H} & 1.330418 & 2.602469 & 1.398012\end{array}$

$\begin{array}{llll}\mathrm{H} & 0.241517 & 3.936173 & 0.951924\end{array}$

$\mathrm{H} \quad 4.275953 \quad-1.325593 \quad 0.566578$

$\mathrm{H} \quad-4.556440 \quad 0.334992 \quad-0.625902$

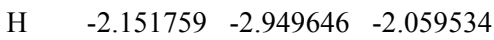

$\begin{array}{llll}\mathrm{H} & 0.128422 & -2.832243 & 0.513209\end{array}$

$\begin{array}{llll}\mathrm{H} & -1.923161 & 1.466107 & 1.743777\end{array}$

$\begin{array}{llll}\mathrm{H} & -3.315521 & 2.132890 & 0.895772\end{array}$

$\begin{array}{llll}\text { O } & 1.716059 & -1.398525 & 1.448859\end{array}$

$\begin{array}{llll}\text { C } & 2.526460 & -2.381547 & 2.045377\end{array}$

$\mathrm{H} \quad 1.895796 \quad-2.906437 \quad 2.773834$

$\begin{array}{llll}\mathrm{H} & 2.897390 & -3.109643 & 1.304053\end{array}$

$\mathrm{H} \quad 3.385001 \quad-1.937228 \quad 2.576673$

H $\quad 0.909267 \quad-2.242925 \quad-0.959808$

$\begin{array}{llll}\text { C } & -3.773771 & 0.521430 & 2.262124\end{array}$

$\begin{array}{llll}\mathrm{H} & -3.953764 & 1.186733 & 3.121300\end{array}$

$\begin{array}{llll}\mathrm{H} & -4.750662 & 0.268091 & 1.822419\end{array}$

$\mathrm{H} \quad-3.326262 \quad-0.410171 \quad 2.643481$

$\mathrm{H} \quad \begin{array}{llll}-0.274333 & -3.561590 & -1.064529\end{array}$ 


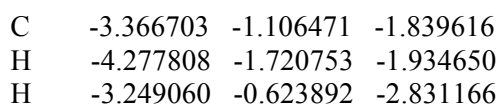




$\begin{array}{rrrr}\mathrm{C} & 1.745629 & -1.032317 & -0.513431 \\ \mathrm{C} & 3.114845 & -1.330174 & -0.491958 \\ \mathrm{C} & 4.051898 & -0.334223 & -0.204418 \\ \mathrm{C} & 3.646562 & 0.978906 & 0.034881 \\ \mathrm{H} & 2.113200 & 3.370667 & -0.444388 \\ \mathrm{H} & 5.114637 & -0.589239 & -0.202277 \\ \mathrm{H} & 4.382972 & 1.769005 & 0.201000 \\ \mathrm{H} & 3.462815 & -2.336384 & -0.727674 \\ \mathrm{C} & -0.485085 & -1.474635 & 1.780984 \\ \mathrm{H} & -0.841272 & -2.436057 & 2.183922 \\ \mathrm{H} & 0.522794 & -1.665715 & 1.397703 \\ \mathrm{C} & -2.435654 & 1.751872 & -1.832150 \\ \mathrm{H} & -1.609973 & 1.609965 & -2.547612 \\ \mathrm{H} & -3.376239 & 1.774667 & -2.400726 \\ \mathrm{H} & -2.304964 & 2.742242 & -1.376541 \\ \mathrm{C} & -0.789215 & 3.094803 & 0.770740 \\ \mathrm{H} & -0.626330 & 4.184095 & 0.733519 \\ \mathrm{H} & -1.854474 & 2.909713 & 0.577302 \\ \mathrm{H} & -0.583421 & 2.758177 & 1.799523 \\ \mathrm{O} & 0.799327 & -1.911801 & -0.894938 \\ \mathrm{C} & 1.155982 & -3.237962 & -1.190265 \\ \mathrm{H} & 1.844369 & -3.297618 & -2.050557 \\ \mathrm{H} & 1.619031 & -3.739588 & -0.323301 \\ \mathrm{H} & 0.225537 & -3.760955 & -1.445456 \\ \mathrm{H} & -4.451825 & 0.060701 & -1.391366 \\ \mathrm{C} & -0.395844 & -0.460558 & 2.922726 \\ \mathrm{H} & -1.382474 & -0.287959 & 3.381146 \\ \mathrm{H} & 0.284311 & -0.825490 & 3.707697 \\ \mathrm{H} & -0.010557 & 0.508887 & 2.573374 \\ \mathrm{H} & -2.883546 & -2.515846 & 1.298577 \\ \mathrm{O} & -3.686966 & -1.283157 & -0.089626\end{array}$

motor $2 \mathrm{~b}$ - syn-(M)-stable- $Z$

$\begin{array}{lrrr}\mathrm{C} & 2.860786 & 1.287833 & -1.216450 \\ \mathrm{C} & 1.349164 & -0.171394 & 0.004952 \\ \mathrm{C} & 3.780077 & -0.494442 & -0.106018 \\ \mathrm{C} & 2.608508 & -0.828821 & 0.463544 \\ \mathrm{C} & 0.146672 & -0.815421 & 0.103826 \\ \mathrm{C} & -1.239683 & -0.365097 & -0.189535 \\ \mathrm{C} & 0.021470 & -2.347149 & 0.112252 \\ \mathrm{C} & -1.897621 & -1.372354 & -0.913547 \\ \mathrm{H} & 0.980200 & -2.825673 & -0.125178 \\ \mathrm{C} & -0.973078 & -2.552532 & -1.060033 \\ \mathrm{H} & -1.503498 & -3.515484 & -1.010432 \\ \mathrm{C} & -1.994485 & 0.714748 & 0.305020 \\ \mathrm{C} & -3.332398 & 0.850775 & -0.097477 \\ \mathrm{C} & -3.929936 & -0.113321 & -0.912368 \\ \mathrm{C} & -3.227140 & -1.252011 & -1.305926 \\ \mathrm{H} & -0.437076 & -2.511998 & -2.023786 \\ \mathrm{H} & -4.975876 & 0.014373 & -1.202344 \\ \mathrm{H} & -3.715871 & -2.042710 & -1.880023 \\ \mathrm{H} & -3.927595 & 1.696860 & 0.246689 \\ \mathrm{C} & 1.633788 & 1.117217 & -0.682204 \\ \mathrm{C} & 2.643329 & -1.758563 & 1.650038 \\ \mathrm{H} & 2.294577 & -2.775706 & 1.425839 \\ \mathrm{C} & 0.668150 & 2.261648 & -0.897921 \\ \mathrm{H} & 0.875307 & 2.713034 & -1.881168 \\ \mathrm{H} & -0.361590 & 1.898171 & -0.947360 \\ \mathrm{H} & 3.124231 & 2.144268 & -1.840647 \\ \mathrm{C} & -0.546012 & -2.937513 & 1.405816 \\ \mathrm{H} & -0.651986 & -4.030546 & 1.319486 \\ \mathrm{H} & 0.099111 & -2.724981 & 2.269354 \\ \mathrm{H} & -1.542209 & -2.520082 & 1.623619 \\ \mathrm{O} & -1.394594 & 1.545298 & 1.179354 \\ \mathrm{C} & -2.123289 & 2.618040 & 1.723314\end{array}$




$\begin{array}{rrrr}\mathrm{H} & -2.449644 & 3.330380 & 0.947147 \\ \mathrm{H} & -3.007298 & 2.267180 & 2.282634 \\ \mathrm{H} & -1.449720 & 3.135246 & 2.417546 \\ \mathrm{H} & 4.741454 & -0.929305 & 0.174668 \\ \mathrm{H} & 3.666534 & -1.844227 & 2.043573 \\ \mathrm{H} & 2.002918 & -1.364930 & 2.454506 \\ \mathrm{C} & 0.810270 & 3.337961 & 0.178917 \\ \mathrm{H} & 1.797237 & 3.822896 & 0.110916 \\ \mathrm{H} & 0.041290 & 4.118515 & 0.068724 \\ \mathrm{H} & 0.715566 & 2.904149 & 1.184011 \\ \mathrm{O} & 3.911157 & 0.450070 & -1.061227\end{array}$

motor $2 \mathrm{~b}$ - anti-(M)-stable- $Z$

$\begin{array}{llll}\mathrm{C} & 2.551440 & -1.806075 & 0.057\end{array}$

$\begin{array}{llll}\text { C } & 1.284550 & 0.279503 & 0.067858\end{array}$

$\begin{array}{llll}\text { C } & 3.603852 & 0.118249 & -0.620959\end{array}$

$\begin{array}{llll}\text { C } & 2.594260 & 0.918085 & -0.221150\end{array}$

$\begin{array}{llll}\text { C } & 0.068323 & 0.893528 & -0.015114\end{array}$

$\begin{array}{lllll}\mathrm{C} & -1.268351 & 0.259550 & -0.018896\end{array}$

$\begin{array}{llll}\text { C } & -0.232563 & 2.396618 & -0.019842\end{array}$

$\begin{array}{llll}\text { C } & -2.224643 & 1.164800 & 0.465310\end{array}$

$\begin{array}{llll}\mathrm{H} & 0.549708 & 2.969060 & 0.488305\end{array}$

$\begin{array}{llll}\text { C } & -1.547352 & 2.468658 & 0.794487\end{array}$

$\begin{array}{llll}\mathrm{H} & -2.163117 & 3.344046 & 0.537020\end{array}$

$\begin{array}{llll}\text { C } & -1.699366 & -0.965494 & -0.565754\end{array}$

$\begin{array}{llll}\text { C } & -3.056506 & -1.310422 & -0.477069\end{array}$

$\begin{array}{llll}\text { C } & -3.975947 & -0.420380 & 0.083496\end{array}$

$\begin{array}{llll}\text { C } & -3.574735 & 0.835748 & 0.540671\end{array}$

$\begin{array}{llll}\mathrm{H} & -1.328030 & 2.526557 & 1.874686\end{array}$

$\mathrm{H} \quad-5.029222 \quad-0.708348 \quad 0.131766$

$\begin{array}{llll}\mathrm{H} & -4.304958 & 1.548915 & 0.930338\end{array}$

$\mathrm{H} \quad-3.410742 \quad-2.263573 \quad-0.871182$

$\begin{array}{llll}\text { C } & 1.457050 & -1.143350 & 0.471551\end{array}$

$\begin{array}{llll}\mathrm{H} & 4.574678 & 0.492650 & -0.952040\end{array}$

$\begin{array}{llll}\text { C } & 2.872654 & 2.395920 & -0.153566\end{array}$

$\begin{array}{llll}\mathrm{H} & 2.271281 & 2.975474 & -0.867423\end{array}$

$\mathrm{H} \quad 2.670178 \quad 2.793648 \quad 0.852590$

$\begin{array}{llll}\mathrm{H} & 3.931511 & 2.590995 & -0.376397\end{array}$

$\begin{array}{llll}\text { C } & 0.586678 & -1.803581 & 1.517324\end{array}$

$\begin{array}{llll}\mathrm{H} & -0.468927 & -1.831305 & 1.222513\end{array}$

$\begin{array}{llll}\text { C } & -0.430357 & 2.957758 & -1.431605\end{array}$

$\mathrm{H} \quad-1.270744 \quad 2.458795 \quad-1.940867$

$\mathrm{H} \quad-0.648023 \quad 4.037118 \quad-1.396394$

$\begin{array}{llll}\mathrm{H} & 0.465812 & 2.809898 & -2.052582\end{array}$

$\begin{array}{llll}\text { O } & -0.772748 & -1.725711 & -1.176774\end{array}$

$\begin{array}{llll}\text { C } & -1.099575 & -3.028001 & -1.590613\end{array}$

$\mathrm{H} \quad-0.175546 \quad-3.471169-1.983375$

$\mathrm{H} \quad-1.461352 \quad-3.644823 \quad-0.750212$

$\mathrm{H} \quad-1.859852 \quad-3.027136 \quad-2.389956$

$\begin{array}{llll}\mathrm{H} & 0.905531 & -2.853537 & 1.615978\end{array}$

$\begin{array}{llll}\text { C } & 0.692286 & -1.113849 & 2.878137\end{array}$

$\begin{array}{llll}\mathrm{H} & 0.074378 & -1.634088 & 3.626148\end{array}$

$\mathrm{H} \quad \begin{array}{llll}1.731807 & -1.103543 & 3.242184\end{array}$

$\begin{array}{llll}\mathrm{H} & 0.342966 & -0.070931 & 2.825295\end{array}$

$\mathrm{H} \quad 2.762486-2.851372 \quad 0.294395$

$\begin{array}{llll}\text { O } & 3.551498 & -1.233154 & -0.652520\end{array}$

motor $2 b-$ TS1

$\begin{array}{rrrr}\mathrm{C} & 2.401909 & -1.210193 & 0.051386 \\ \mathrm{C} & 3.105760 & -1.131147 & -1.090681 \\ \mathrm{C} & 1.205788 & -0.305527 & 0.092745 \\ \mathrm{C} & 2.361902 & 0.995920 & -1.527546 \\ \mathrm{C} & 1.625928 & 1.040682 & -0.402228 \\ \mathrm{C} & -0.047224 & -0.805229 & 0.253517\end{array}$ 


\begin{tabular}{rrrr}
$\mathrm{C}$ & -1.405274 & -0.247389 & -0.031472 \\
$\mathrm{C}$ & -0.288469 & -2.301329 & 0.476481 \\
$\mathrm{C}$ & -2.225783 & -1.299306 & -0.481758 \\
$\mathrm{H}$ & 0.583387 & -2.889565 & 0.165545 \\
$\mathrm{C}$ & -1.481626 & -2.603783 & -0.445012 \\
$\mathrm{H}$ & -2.107091 & -3.435256 & -0.085671 \\
$\mathrm{C}$ & -1.981844 & 1.035610 & 0.077981 \\
$\mathrm{C}$ & -3.305283 & 1.239487 & -0.343422 \\
$\mathrm{C}$ & -4.071353 & 0.181391 & -0.831133 \\
$\mathrm{C}$ & -3.541493 & -1.104237 & -0.891472 \\
$\mathrm{H}$ & -1.131821 & -2.867315 & -1.457682 \\
$\mathrm{H}$ & -5.101469 & 0.369600 & -1.143511 \\
$\mathrm{H}$ & -4.143163 & -1.945881 & -1.242144 \\
$\mathrm{H}$ & -3.755945 & 2.228979 & -0.270508 \\
$\mathrm{C}$ & 1.516996 & 2.335435 & 0.378796 \\
$\mathrm{H}$ & 0.824741 & 2.188775 & 1.211979 \\
$\mathrm{H}$ & 1.078964 & 3.125190 & -0.253066 \\
$\mathrm{C}$ & 2.863037 & -2.063511 & 1.200148 \\
$\mathrm{H}$ & 3.062328 & -1.428210 & 2.078826 \\
$\mathrm{C}$ & -0.616638 & -2.614620 & 1.939420 \\
$\mathrm{H}$ & -0.779311 & -3.694573 & 2.081339 \\
$\mathrm{H}$ & 0.196010 & -2.299183 & 2.610857 \\
$\mathrm{H}$ & -1.532893 & -2.090708 & 2.256821 \\
$\mathrm{H}$ & 2.710980 & 1.870604 & -2.082923 \\
$\mathrm{O}$ & -1.245892 & 2.011952 & 0.630633 \\
$\mathrm{C}$ & -1.766995 & 3.311459 & 0.756419 \\
$\mathrm{H}$ & -2.008279 & 3.752713 & -0.225042 \\
$\mathrm{H}$ & -2.665154 & 3.332497 & 1.396567 \\
$\mathrm{H}$ & -0.983397 & 3.914441 & 1.232966 \\
$\mathrm{H}$ & 3.958970 & -1.762300 & -1.350760 \\
$\mathrm{H}$ & 3.797855 & -2.585421 & 0.947264 \\
$\mathrm{H}$ & 2.127235 & -2.816663 & 1.511955 \\
$\mathrm{C}$ & 2.869893 & 2.802450 & 0.919706 \\
$\mathrm{H}$ & 3.320144 & 2.036992 & 1.572078 \\
$\mathrm{H}$ & 3.584993 & 3.010839 & 0.108843 \\
$\mathrm{H}$ & 2.755193 & 3.724142 & 1.512695 \\
$\mathrm{O}$ & 2.857433 & -0.171537 & -2.029093 \\
& & & \\
\hline
\end{tabular}

motor 2b-TS2

$\begin{array}{llll}\text { C } & -0.656657 & -2.204197 & -0.976029\end{array}$

$\begin{array}{llll}\text { C } & -1.645034 & -1.096427 & -0.671353\end{array}$

$\begin{array}{llll}\text { C } & -2.895412 & -1.309003 & -1.131670\end{array}$

$\begin{array}{llll}\text { C } & -1.350459 & 0.202863 & -0.010521\end{array}$

$\begin{array}{llll}\text { C } & -3.793077 & 0.488425 & -0.029714\end{array}$

$\begin{array}{llll}\text { C } & -2.599272 & 0.872384 & 0.457569\end{array}$

$\begin{array}{llll}\text { C } & -2.602014 & 1.868582 & 1.588741\end{array}$

$\begin{array}{llll}\text { C } & -0.139285 & 0.836265 & 0.069272\end{array}$

$\begin{array}{llll}\text { C } & 1.241154 & 0.352062 & -0.190462\end{array}$

$\begin{array}{llll}\text { C } & 0.017007 & 2.365590 & 0.032624\end{array}$

$\begin{array}{llll}\mathrm{C} & 1.933845 & 1.324605 & -0.928571\end{array}$

$\begin{array}{llll}\mathrm{H} & -0.928007 & 2.856608 & -0.232717\end{array}$

$\begin{array}{llll}\text { C } & 1.032065 & 2.514099 & -1.131449\end{array}$

$\begin{array}{llll}\mathrm{H} & 1.577031 & 3.469877 & -1.107587\end{array}$

$\begin{array}{llll}\text { C } & 1.961066 & -0.729179 & 0.350867\end{array}$

$\begin{array}{llll}\text { C } & 3.306666 & -0.899237 & -0.010218\end{array}$

$\begin{array}{llll}\text { C } & 3.942318 & 0.031062 & -0.835874\end{array}$

$\begin{array}{llll}\text { C } & 3.270491 & 1.168808 & -1.282989\end{array}$

$\mathrm{H} \quad \begin{array}{llll}0.509355 & 2.447992 & -2.101084\end{array}$

$\mathrm{H} \quad 4.993187 \quad-0.122660 \quad-1.093549$

$\begin{array}{llll}\mathrm{H} & 3.788378 & 1.931930 & -1.868715\end{array}$

$\begin{array}{llll}\text { C } & 0.584262 & 2.986745 & 1.312286\end{array}$

$\begin{array}{llll}\mathrm{H} & -0.074917 & 2.822237 & 2.175242\end{array}$

$\begin{array}{llll}\mathrm{H} & 1.566976 & 2.552375 & 1.556303\end{array}$

$\begin{array}{llll}\mathrm{H} & 0.719536 & 4.072895 & 1.188326\end{array}$

$\begin{array}{llll}\mathrm{H} & 3.877077 & -1.745643 & 0.372868\end{array}$ 


$\begin{array}{lrrr}\mathrm{H} & -4.745102 & 0.922950 & 0.281397 \\ \mathrm{H} & -3.165022 & -2.177287 & -1.736452 \\ \mathrm{H} & 0.363759 & -1.814284 & -1.003870 \\ \mathrm{H} & -3.614116 & 1.976442 & 2.004671 \\ \mathrm{H} & -1.939706 & 1.520189 & 2.396343 \\ \mathrm{H} & -2.260806 & 2.870952 & 1.296644 \\ \mathrm{O} & 1.318489 & -1.528457 & 1.224011 \\ \mathrm{C} & 2.016419 & -2.590351 & 1.826726 \\ \mathrm{H} & 1.314859 & -3.078370 & 2.514559 \\ \mathrm{H} & 2.360835 & -3.330348 & 1.085042 \\ \mathrm{H} & 2.884796 & -2.230108 & 2.404238 \\ \mathrm{H} & -0.857734 & -2.581777 & -1.991314 \\ \mathrm{C} & -0.764142 & -3.367244 & 0.010593 \\ \mathrm{H} & -1.736828 & -3.874470 & -0.092630 \\ \mathrm{H} & 0.025851 & -4.114141 & -0.165115 \\ \mathrm{H} & -0.678230 & -3.013788 & 1.047049 \\ \mathrm{O} & -3.961091 & -0.503627 & -0.927588\end{array}$

motor $2 b-$ TS3

$\begin{array}{lrrr}\mathrm{C} & 2.367292 & -0.992538 & -0.440060 \\ \mathrm{C} & 3.222135 & -1.038888 & 0.592334 \\ \mathrm{C} & 1.116793 & -0.209723 & -0.185072 \\ \mathrm{C} & 2.427074 & 0.938691 & 1.422100 \\ \mathrm{C} & 1.557356 & 1.099546 & 0.404578 \\ \mathrm{C} & -0.104636 & -0.813542 & -0.251151 \\ \mathrm{C} & -1.499897 & -0.305530 & -0.037233 \\ \mathrm{C} & -0.260646 & -2.340897 & -0.501446 \\ \mathrm{C} & -2.382749 & -1.388223 & 0.125345 \\ \mathrm{H} & -0.274114 & -2.500792 & -1.595236 \\ \mathrm{C} & -1.658198 & -2.687484 & 0.041256 \\ \mathrm{H} & -1.572925 & -3.129018 & 1.049694 \\ \mathrm{C} & -2.079129 & 0.987100 & -0.041543 \\ \mathrm{C} & -3.453547 & 1.143322 & 0.195950 \\ \mathrm{C} & -4.280699 & 0.040271 & 0.396955 \\ \mathrm{C} & -3.749544 & -1.242324 & 0.347392 \\ \mathrm{H} & -2.184667 & -3.425209 & -0.582988 \\ \mathrm{H} & -5.347551 & 0.195651 & 0.574409 \\ \mathrm{H} & -4.383037 & -2.123256 & 0.473342 \\ \mathrm{H} & -3.891668 & 2.140492 & 0.209530 \\ \mathrm{O} & -1.293392 & 2.037327 & -0.313770 \\ \mathrm{C} & -1.851749 & 3.321350 & -0.445196 \\ \mathrm{H} & -2.627832 & 3.348189 & -1.227975 \\ \mathrm{H} & -2.279347 & 3.683260 & 0.504662 \\ \mathrm{H} & -1.033390 & 3.990799 & -0.738812 \\ \mathrm{H} & 4.141943 & -1.626786 & 0.640143 \\ \mathrm{C} & 1.359094 & 2.487937 & -0.179793 \\ \mathrm{H} & 0.759806 & 2.393438 & -1.090405 \\ \mathrm{H} & 0.773239 & 3.117661 & 0.506975 \\ \mathrm{C} & 2.677433 & -1.582866 & -1.784939 \\ \mathrm{H} & 2.913518 & -0.775748 & -2.498122 \\ \mathrm{H} & 3.550936 & -2.250462 & -1.737280 \\ \mathrm{H} & 1.832326 & -2.142694 & -2.210669 \\ \mathrm{C} & 0.739079 & -3.320157 & 0.123525 \\ \mathrm{H} & 1.708739 & -3.374366 & -0.379407 \\ \mathrm{H} & 0.914677 & -3.080367 & 1.184004 \\ \mathrm{H} & 0.303093 & -4.331149 & 0.078708 \\ \mathrm{C} & 2.674583 & 3.191708 & -0.517322 \\ \mathrm{H} & 2.480222 & 4.156749 & -1.012736 \\ \mathrm{H} & 3.276741 & 3.397761 & 0.380592 \\ \mathrm{H} & 3.289768 & 2.580965 & -1.197445 \\ \mathrm{H} & 2.815589 & 1.748975 & 2.044332 \\ \mathrm{O} & 3.032600 & -0.252403 & 1.692596\end{array}$

motor $2 \mathrm{~b}$ - $(P)$-unstable- $E$ 


$\begin{array}{lrrr}\mathrm{C} & 2.429429 & 0.408456 & 0.338660 \\ \mathrm{C} & 3.531830 & -0.349918 & 0.162013 \\ \mathrm{C} & 1.160522 & 0.008961 & -0.302386 \\ \mathrm{C} & 2.461018 & -1.828789 & -1.244633 \\ \mathrm{C} & 1.262718 & -1.223779 & -1.109463 \\ \mathrm{C} & 0.002321 & 0.747009 & -0.198777 \\ \mathrm{C} & -1.374301 & 0.245036 & -0.131298 \\ \mathrm{C} & -0.087565 & 2.279910 & -0.186278 \\ \mathrm{C} & -2.293068 & 1.285236 & -0.344772 \\ \mathrm{H} & 0.039193 & 2.676893 & 0.838641 \\ \mathrm{C} & -1.550112 & 2.559309 & -0.630194 \\ \mathrm{H} & -1.574197 & 2.777914 & -1.712663 \\ \mathrm{C} & -1.853921 & -0.991857 & 0.356032 \\ \mathrm{C} & -3.237230 & -1.200571 & 0.446354 \\ \mathrm{C} & -4.131253 & -0.173412 & 0.132151 \\ \mathrm{C} & -3.666614 & 1.088680 & -0.240030 \\ \mathrm{H} & -1.993671 & 3.428059 & -0.121228 \\ \mathrm{H} & -5.204606 & -0.359724 & 0.218408 \\ \mathrm{H} & -4.364851 & 1.908625 & -0.425047 \\ \mathrm{H} & -3.628005 & -2.158131 & 0.791769 \\ \mathrm{C} & 0.160936 & -1.679244 & -2.027796 \\ \mathrm{H} & -0.669827 & -2.162992 & -1.500869 \\ \mathrm{H} & -0.254741 & -0.814988 & -2.569986 \\ \mathrm{H} & 0.547410 & -2.393683 & -2.768942 \\ \mathrm{C} & 2.495777 & 1.444049 & 1.442133 \\ \mathrm{H} & 2.133853 & 2.423511 & 1.103742 \\ \mathrm{C} & 0.895994 & 2.978057 & -1.125328 \\ \mathrm{H} & 0.741821 & 4.068881 & -1.102932 \\ \mathrm{H} & 1.948645 & 2.786804 & -0.876782 \\ \mathrm{H} & 0.738948 & 2.635995 & -2.161127 \\ \mathrm{H} & 2.640418 & -2.671468 & -1.914343 \\ \mathrm{O} & -0.936987 & -1.894661 & 0.759477 \\ \mathrm{C} & -1.356564 & -3.147562 & 1.237043 \\ \mathrm{H} & -1.984563 & -3.054637 & 2.139577 \\ \mathrm{H} & -1.910445 & -3.714841 & 0.469443 \\ \mathrm{H} & -0.445797 & -3.702009 & 1.496687 \\ \mathrm{H} & 4.475925 & -0.173771 & 0.681101 \\ \mathrm{H} & 3.552938 & 1.595089 & 1.711431 \\ \mathrm{C} & 1.714964 & 1.025290 & 2.689428 \\ \mathrm{H} & 0.650257 & 0.860710 & 2.465804 \\ \mathrm{H} & 2.116406 & 0.089232 & 3.108625 \\ \mathrm{H} & 1.780576 & 1.802339 & 3.466536 \\ \mathrm{O} & 3.596365 & -1.442461 & -0.623850\end{array}$

motor 2b - syn-(M)-stable- $E$

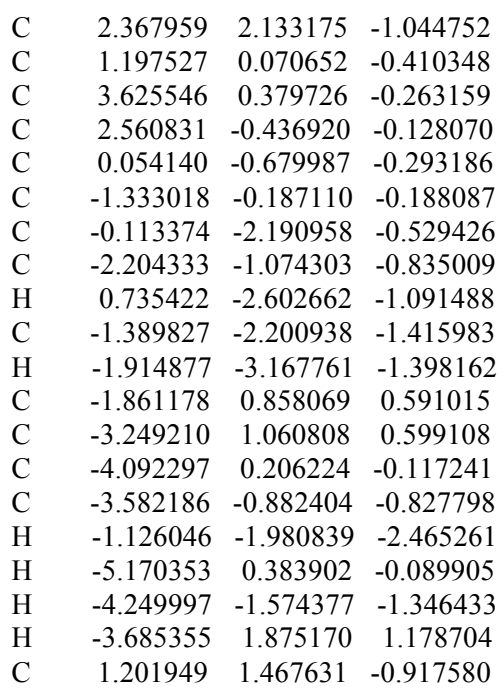




$\begin{array}{lrrr}\mathrm{C} & 2.842571 & -1.745691 & 0.579424 \\ \mathrm{H} & 2.257590 & -2.569576 & 0.157325 \\ \mathrm{C} & 0.002430 & 2.122148 & -1.553156 \\ \mathrm{H} & -0.732636 & 2.479565 & -0.823155 \\ \mathrm{H} & 0.318363 & 2.986051 & -2.155434 \\ \mathrm{H} & -0.512487 & 1.417322 & -2.223453 \\ \mathrm{H} & 2.446610 & 3.126197 & -1.490585 \\ \mathrm{C} & -0.361777 & -3.031929 & 0.727114 \\ \mathrm{H} & -0.568367 & -4.079059 & 0.454664 \\ \mathrm{H} & 0.495058 & -3.029619 & 1.412517 \\ \mathrm{H} & -1.233579 & -2.652783 & 1.284322 \\ \mathrm{O} & -0.987911 & 1.591873 & 1.311633 \\ \mathrm{C} & -1.464659 & 2.657258 & 2.095104 \\ \mathrm{H} & -1.958040 & 3.429425 & 1.480024 \\ \mathrm{H} & -2.168944 & 2.312314 & 2.871145 \\ \mathrm{H} & -0.589051 & 3.102099 & 2.584292 \\ \mathrm{H} & 4.641665 & 0.086431 & 0.007877 \\ \mathrm{H} & 3.896770 & -2.014192 & 0.409231 \\ \mathrm{C} & 2.594887 & -1.637646 & 2.086452 \\ \mathrm{H} & 2.726793 & -2.611659 & 2.582813 \\ \mathrm{H} & 3.300778 & -0.925237 & 2.541379 \\ \mathrm{H} & 1.577449 & -1.278818 & 2.304020 \\ \mathrm{O} & 3.580081 & 1.653286 & -0.700503\end{array}$

motor $2 \mathrm{~b}$ - anti-(M)-stable- $E$

$\begin{array}{lrrr}\mathrm{C} & -1.868728 & 2.486693 & 0.262674 \\ \mathrm{C} & -1.156546 & 0.162464 & 0.132288 \\ \mathrm{C} & -3.300456 & 0.950482 & -0.665911 \\ \mathrm{C} & -2.563290 & -0.107698 & -0.271493 \\ \mathrm{C} & -0.107126 & -0.696892 & -0.016209 \\ \mathrm{C} & 1.341227 & -0.399340 & 0.075879 \\ \mathrm{C} & -0.163374 & -2.203779 & -0.286418 \\ \mathrm{C} & 2.051214 & -1.574748 & 0.366032 \\ \mathrm{H} & -1.059029 & -2.661692 & 0.144573 \\ \mathrm{C} & 1.089535 & -2.729295 & 0.452414 \\ \mathrm{H} & 1.495209 & -3.650756 & 0.007630 \\ \mathrm{C} & 2.061161 & 0.772086 & -0.235896 \\ \mathrm{C} & 3.457091 & 0.773129 & -0.095775 \\ \mathrm{C} & 4.130537 & -0.392733 & 0.275303 \\ \mathrm{C} & 3.437832 & -1.585281 & 0.487709 \\ \mathrm{H} & 0.849665 & -2.949735 & 1.507065 \\ \mathrm{H} & 5.219339 & -0.368372 & 0.367352 \\ \mathrm{H} & 3.971836 & -2.508411 & 0.725052 \\ \mathrm{H} & 4.029591 & 1.677037 & -0.306960 \\ \mathrm{C} & -1.025855 & 1.533899 & 0.695523 \\ \mathrm{C} & -3.239009 & -1.458742 & -0.257092 \\ \mathrm{H} & -2.697708 & -2.180100 & -0.885205 \\ \mathrm{C} & -0.124132 & 1.836457 & 1.860805 \\ \mathrm{H} & 0.907341 & 1.488876 & 1.732602 \\ \mathrm{H} & -0.098310 & 2.917405 & 2.063387 \\ \mathrm{H} & -0.515401 & 1.337032 & 2.763193 \\ \mathrm{H} & -1.843038 & 3.529122 & 0.587504 \\ \mathrm{C} & -0.110867 & -2.525070 & -1.782953 \\ \mathrm{H} & -0.141990 & -3.612995 & -1.952806 \\ \mathrm{H} & -0.959864 & -2.071438 & -2.316952 \\ \mathrm{H} & 0.813852 & -2.135428 & -2.238363 \\ \mathrm{O} & 1.359140 & 1.823778 & -0.691651 \\ \mathrm{C} & 1.980692 & 3.074877 & -0.836007 \\ \mathrm{H} & 2.440622 & 3.413463 & 0.108177 \\ \mathrm{H} & 2.749944 & 3.064364 & -1.626700 \\ \mathrm{H} & 1.192254 & 3.783359 & -1.120750 \\ \mathrm{H} & -4.309411 & 0.864173 & -1.075071 \\ \mathrm{C} & -3.411730 & -2.018145 & 1.155460 \\ \mathrm{H} & -4.051845 & -1.358252 & 1.761555 \\ \mathrm{H} & -3.878266 & -3.014758 & 1.128490\end{array}$




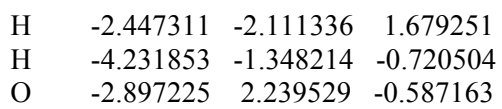

motor $2 b-$ TS4

$\begin{array}{lrrc}\mathrm{C} & -2.503171 & -0.015269 & 0.083898 \\ \mathrm{C} & -3.019223 & 0.501576 & 1.213078 \\ \mathrm{C} & -1.067543 & 0.368787 & -0.137220 \\ \mathrm{C} & -1.539986 & 2.260013 & 1.217298 \\ \mathrm{C} & -0.946988 & 1.840446 & 0.086453 \\ \mathrm{C} & -0.081679 & -0.563735 & -0.196709 \\ \mathrm{C} & 1.397507 & -0.489617 & 0.016262 \\ \mathrm{C} & -0.399160 & -2.062411 & -0.220898 \\ \mathrm{C} & 1.828059 & -1.717196 & 0.556012 \\ \mathrm{H} & -1.391733 & -2.254176 & 0.204299 \\ \mathrm{C} & 0.683633 & -2.681865 & 0.674827 \\ \mathrm{H} & 0.960479 & -3.703900 & 0.373791 \\ \mathrm{C} & 2.370692 & 0.514831 & -0.180685 \\ \mathrm{C} & 3.699234 & 0.281000 & 0.206907 \\ \mathrm{C} & 4.080629 & -0.939602 & 0.762347 \\ \mathrm{C} & 3.146360 & -1.955559 & 0.934510 \\ \mathrm{H} & 0.343609 & -2.731039 & 1.723199 \\ \mathrm{H} & 5.124401 & -1.092782 & 1.047258 \\ \mathrm{H} & 3.434619 & -2.921672 & 1.354960 \\ \mathrm{H} & 4.452869 & 1.054790 & 0.063254 \\ \mathrm{C} & -0.489596 & 2.832174 & -0.955101 \\ \mathrm{H} & 0.108060 & 2.353935 & -1.734962 \\ \mathrm{H} & -1.371093 & 3.290306 & -1.432994 \\ \mathrm{H} & 0.103113 & 3.646806 & -0.512983 \\ \mathrm{C} & -3.324572 & -0.804048 & -0.908862 \\ \mathrm{H} & -2.874855 & -1.791418 & -1.085788 \\ \mathrm{C} & -0.337669 & -2.631626 & -1.642320 \\ \mathrm{H} & -0.585906 & -3.704818 & -1.642317 \\ \mathrm{H} & -1.039810 & -2.117026 & -2.314701 \\ \mathrm{H} & 0.672435 & -2.518830 & -2.068564 \\ \mathrm{H} & -1.528021 & 3.286604 & 1.593373 \\ \mathrm{O} & 1.990620 & 1.658536 & -0.770529 \\ \mathrm{C} & 2.926222 & 2.683855 & -0.992602 \\ \mathrm{H} & 3.344082 & 3.068641 & -0.047288 \\ \mathrm{H} & 3.749804 & 2.352478 & -1.647044 \\ \mathrm{H} & 2.385820 & 3.495511 & -1.496217 \\ \mathrm{H} & -4.009121 & 0.290508 & 1.620603 \\ \mathrm{H} & -3.248866 & -0.278449 & -1.877080 \\ \mathrm{C} & -4.792713 & -0.987404 & -0.546584 \\ \mathrm{H} & -4.912364 & -1.554619 & 0.389872 \\ \mathrm{H} & -5.305817 & -0.020752 & -0.424044 \\ \mathrm{H} & -5.313142 & -1.544730 & -1.339664 \\ \mathrm{O} & -2.350466 & 1.440030 & 1.949200\end{array}$

\section{motor $2 b-$ TS5}

$\begin{array}{lllll}\text { C } & & 0.095651 & -1.771730 & -1.904993\end{array}$

$\begin{array}{llll}\text { C } & -0.901208 & -1.493210 & -0.812106\end{array}$

$\begin{array}{lllll}\text { C } & -1.765198 & -2.466632 & -0.476588\end{array}$

$\begin{array}{llll}\text { C } & -1.080507 & -0.129145 & -0.241187\end{array}$

$\begin{array}{llll}\text { C } & -3.227762 & -0.982551 & 0.483586\end{array}$

$\begin{array}{llll}\text { C } & -2.491929 & 0.102681 & 0.162300\end{array}$

$\begin{array}{llll}\text { C } & -3.161763 & 1.454968 & 0.356314\end{array}$

$\begin{array}{llll}\text { C } & -0.035830 & 0.747343 & -0.115796\end{array}$

$\begin{array}{llll}\text { C } & 1.404928 & 0.411945 & -0.109939\end{array}$

$\begin{array}{llll}\text { C } & -0.065001 & 2.277574 & 0.003385\end{array}$

$\begin{array}{llll}\text { C } & 2.166255 & 1.536927 & -0.461425\end{array}$

$\begin{array}{llll}\mathrm{H} & -0.912652 & 2.712143 & -0.537273\end{array}$

$\begin{array}{llll}\text { C } & 1.243483 & 2.698487 & -0.709552\end{array}$

H $\quad 1.648151 \quad 3.649072-0.329496$ 


$\begin{array}{rrrr}\mathrm{C} & 2.071232 & -0.741447 & 0.353827 \\ \mathrm{C} & 3.472140 & -0.790272 & 0.296737 \\ \mathrm{C} & 4.201017 & 0.318444 & -0.139919 \\ \mathrm{C} & 3.556912 & 1.503009 & -0.499568 \\ \mathrm{H} & 1.066693 & 2.826018 & -1.791438 \\ \mathrm{H} & 5.292015 & 0.258163 & -0.165279 \\ \mathrm{H} & 4.130757 & 2.386860 & -0.788306 \\ \mathrm{C} & -0.092533 & 2.757870 & 1.456422 \\ \mathrm{H} & -0.989782 & 2.394333 & 1.978567 \\ \mathrm{H} & 0.787782 & 2.387066 & 2.005929 \\ \mathrm{H} & -0.087465 & 3.858266 & 1.509516 \\ \mathrm{H} & 4.004660 & -1.684690 & 0.621833 \\ \mathrm{H} & -4.226899 & -0.923926 & 0.918622 \\ \mathrm{H} & -1.712326 & -3.492580 & -0.846640 \\ \mathrm{H} & 1.143785 & -1.717447 & -1.586925 \\ \mathrm{H} & -3.264744 & 1.661009 & 1.435218 \\ \mathrm{H} & -2.522418 & 2.247343 & -0.036078 \\ \mathrm{O} & 1.312361 & -1.728778 & 0.862380 \\ \mathrm{C} & 1.892837 & -2.970109 & 1.169163 \\ \mathrm{H} & 1.074209 & -3.627165 & 1.490101 \\ \mathrm{H} & 2.385063 & -3.419007 & 0.289452 \\ \mathrm{H} & 2.626611 & -2.892323 & 1.989591 \\ \mathrm{H} & -0.025210 & -1.032475 & -2.713796 \\ \mathrm{C} & -4.527385 & 1.581567 & -0.319574 \\ \mathrm{H} & -4.933867 & 2.594988 & -0.177317 \\ \mathrm{H} & -4.447855 & 1.397842 & -1.402668 \\ \mathrm{H} & -5.265881 & 0.873527 & 0.085500 \\ \mathrm{H} & -0.071605 & -2.770180 & -2.334819 \\ \mathrm{O} & -2.841244 & -2.266366 & 0.317518\end{array}$

motor 2b - TS6

$\begin{array}{rrrr}\mathrm{C} & 2.430397 & 0.067322 & -0.202464 \\ \mathrm{C} & 3.172665 & 0.439956 & 0.851646 \\ \mathrm{C} & 0.976096 & 0.420529 & -0.103504 \\ \mathrm{C} & 1.725836 & 2.138225 & 1.351232 \\ \mathrm{C} & 0.925724 & 1.863935 & 0.302619 \\ \mathrm{C} & 0.021382 & -0.554464 & -0.134922 \\ \mathrm{C} & -1.476870 & -0.521019 & -0.045267 \\ \mathrm{C} & 0.400745 & -2.062866 & -0.187336 \\ \mathrm{C} & -1.972218 & -1.822123 & 0.158539 \\ \mathrm{H} & 0.602710 & -2.324107 & -1.241202 \\ \mathrm{C} & -0.866384 & -2.816411 & 0.249023 \\ \mathrm{H} & -0.767511 & -3.156193 & 1.294602 \\ \mathrm{C} & -2.444207 & 0.507864 & -0.171792 \\ \mathrm{C} & -3.808841 & 0.212982 & -0.027054 \\ \mathrm{C} & -4.247091 & -1.088682 & 0.205930 \\ \mathrm{C} & -3.325447 & -2.123491 & 0.289860 \\ \mathrm{H} & -1.055647 & -3.715883 & -0.356360 \\ \mathrm{H} & -5.317094 & -1.283790 & 0.310358 \\ \mathrm{H} & -3.644145 & -3.155298 & 0.454321 \\ \mathrm{H} & -4.547653 & 1.008779 & -0.110026 \\ \mathrm{O} & -2.028474 & 1.749268 & -0.457968 \\ \mathrm{C} & -2.969935 & 2.774942 & -0.659690 \\ \mathrm{H} & -3.674060 & 2.528685 & -1.471388 \\ \mathrm{H} & -3.535829 & 2.996779 & 0.260392 \\ \mathrm{H} & -2.404309 & 3.669480 & -0.948682 \\ \mathrm{H} & 1.792927 & 3.114673 & 1.838967 \\ \mathrm{C} & 2.998485 & -0.551239 & -1.455411 \\ \mathrm{H} & 2.843188 & 0.178743 & -2.269452 \\ \mathrm{H} & 2.405611 & -1.431572 & -1.747931 \\ \mathrm{C} & 0.318348 & 3.023608 & -0.455339 \\ \mathrm{H} & -0.152534 & 2.687625 & -1.383446 \\ \mathrm{H} & -0.439012 & 3.557114 & 0.134990 \\ \mathrm{H} & 1.106068 & 3.747188 & -0.715168 \\ \mathrm{C} & 1.568330 & -2.558056 & 0.674175\end{array}$




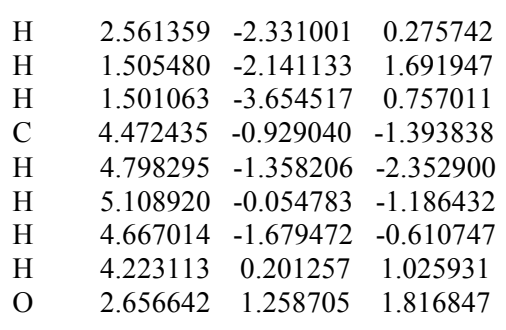

motor 2c - (P)-unstable- $Z$

$\begin{array}{lllll}\text { C } & -3.345247 & 0.503371 & -0.920580\end{array}$

$\begin{array}{lllll}\mathrm{C} & -0.985984 & 0.401904 & -0.114715\end{array}$

S $\quad \begin{array}{lllll}-3.917386 & -0.950692 & -0.170168\end{array}$

$\begin{array}{lllll}\text { C } & -2.509781 & -1.392798 & 0.741922\end{array}$

$\begin{array}{llll}\text { C } & -1.302214 & -0.783940 & 0.724312\end{array}$

$\begin{array}{lllll}\text { C } & 0.303055 & 0.901551 & -0.186429\end{array}$

$\begin{array}{lllll}\text { C } & 1.550721 & 0.129707 & -0.183075\end{array}$

$\begin{array}{lllll}\text { C } & 0.702021 & 2.389305 & -0.190832\end{array}$

$\begin{array}{llll}\text { C } & 2.662518 & 0.970001 & -0.016337\end{array}$

H $\quad 0.590619 \quad 2.833601 \quad-1.192666$

$\begin{array}{llll}\text { C } & 2.211953 & 2.389479 & 0.166888\end{array}$

$\begin{array}{llll}\mathrm{H} & 2.355580 & 2.701409 & 1.215948\end{array}$

$\begin{array}{lllll}\text { C } & & 1.771560 & -1.225611 & -0.526607\end{array}$

$\begin{array}{llll}\text { C } & 3.081469 & -1.721203 & -0.555774\end{array}$

$\begin{array}{lllll}\text { C } & 4.164505 & -0.872405 & -0.311656\end{array}$

$\begin{array}{lllll}\text { C } & 3.966593 & 0.484797 & -0.059786\end{array}$

$\mathrm{H} \quad 2.796164 \quad 3.087819 \quad-0.451062$

H $\quad \begin{array}{llll}5.176836 & -1.282284 & -0.348249\end{array}$

$\begin{array}{llll}\mathrm{H} & 4.815640 & 1.156901 & 0.085221\end{array}$

$\begin{array}{lllll}\mathrm{H} & 3.267058 & -2.769430 & -0.791897\end{array}$

$\begin{array}{lllll}\text { C } & -2.103799 & 1.042390 & -0.847590\end{array}$

$\begin{array}{lllll}\text { C } & -0.369960 & -1.199730 & 1.852674\end{array}$

H $\quad-0.693888 \quad-2.185181 \quad 2.221090$

H $\quad 0.663191 \quad-1.329353 \quad 1.516187$

$\begin{array}{lllll}\text { C } & -1.847154 & 2.183749 & -1.806940\end{array}$

$\mathrm{H} \quad-1.015480 \quad 1.925047 \quad-2.480772$

$\mathrm{H} \quad \begin{array}{llll}-2.736273 & 2.368064 & -2.424614\end{array}$

H $\quad-1.591406 \quad 3.130864 \quad-1.315432$

$\begin{array}{lllll}\mathrm{H} & -4.096177 & 0.948579 & -1.578734\end{array}$

$\begin{array}{llll}\text { C } & -0.086805 & 3.228359 & 0.817402\end{array}$

$\begin{array}{lllll}\mathrm{H} & 0.220959 & 4.285148 & 0.766070\end{array}$

H $\quad-1.172849 \quad 3.186619 \quad 0.658797$

H $\quad 0.104822 \quad 2.872500 \quad 1.841968$

$\begin{array}{lllll}\mathrm{O} & 0.690518 & -1.965122 & -0.829968\end{array}$

$\begin{array}{lllll}\text { C } & 0.820795 & -3.350947 & -1.021184\end{array}$

H $\quad 1.437023 \quad-3.590210 \quad-1.904392$

$\mathrm{H} \quad \begin{array}{llll}1.253837 & -3.843955 & -0.134296\end{array}$

H $\quad-0.192768 \quad-3.739939 \quad-1.182047$

H $\quad-2.706665 \quad-2.205229 \quad 1.447495$

$\begin{array}{llll}\text { C } & -0.381127 & -0.205824 & 3.015012\end{array}$

$\mathrm{H} \quad-1.390864 \quad-0.098565 \quad 3.441715$

H $\quad 0.292938 \quad-0.544231 \quad 3.817046$

$\begin{array}{llll}\mathrm{H} & -0.042983 & 0.790952 & 2.694811\end{array}$

motor 2c - syn-(M)-stable- $Z$

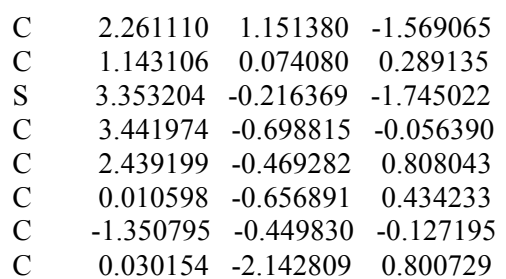




$\begin{array}{lrrr}\mathrm{C} & -1.765933 & -1.662819 & -0.699604 \\ \mathrm{H} & 1.057873 & -2.529451 & 0.831474 \\ \mathrm{C} & -0.735572 & -2.727892 & -0.414711 \\ \mathrm{H} & -1.190127 & -3.709120 & -0.210796 \\ \mathrm{C} & -2.268397 & 0.608500 & -0.023843 \\ \mathrm{C} & -3.503694 & 0.497742 & -0.682472 \\ \mathrm{C} & -3.844951 & -0.679573 & -1.350732 \\ \mathrm{C} & -2.995252 & -1.786455 & -1.339197 \\ \mathrm{H} & -0.047062 & -2.843566 & -1.268941 \\ \mathrm{H} & -4.816059 & -0.741703 & -1.848052 \\ \mathrm{H} & -3.298251 & -2.731254 & -1.796018 \\ \mathrm{H} & -4.222161 & 1.317090 & -0.651627 \\ \mathrm{C} & 1.337722 & 1.268254 & -0.594342 \\ \mathrm{C} & 2.605098 & -0.684262 & 2.287460 \\ \mathrm{H} & 1.847273 & -1.371065 & 2.691216 \\ \mathrm{C} & 0.622938 & 2.584585 & -0.408272 \\ \mathrm{H} & 1.023856 & 3.299904 & -1.142581 \\ \mathrm{H} & -0.444711 & 2.465507 & -0.630019 \\ \mathrm{H} & 2.432862 & 1.951877 & -2.294877 \\ \mathrm{C} & -0.677376 & -2.471478 & 2.116778 \\ \mathrm{H} & -0.654920 & -3.555997 & 2.305522 \\ \mathrm{H} & -0.198350 & -1.969148 & 2.970476 \\ \mathrm{H} & -1.732555 & -2.155607 & 2.094440 \\ \mathrm{O} & -1.935677 & 1.647496 & 0.764669 \\ \mathrm{C} & -2.819250 & 2.734418 & 0.897630 \\ \mathrm{H} & -2.991212 & 3.242888 & -0.066091 \\ \mathrm{H} & -3.788495 & 2.421361 & 1.320723 \\ \mathrm{H} & -2.343548 & 3.439070 & 1.590917 \\ \mathrm{H} & 4.397724 & -1.134325 & 0.249682 \\ \mathrm{H} & 3.599951 & -1.087381 & 2.525215 \\ \mathrm{H} & 2.484064 & 0.272212 & 2.821999 \\ \mathrm{C} & 0.779900 & 3.147502 & 1.001607 \\ \mathrm{H} & 1.840572 & 3.319923 & 1.245002 \\ \mathrm{H} & 0.251231 & 4.108218 & 1.099281 \\ \mathrm{H} & 0.362717 & 2.454377 & 1.747299\end{array}$

motor $2 \mathrm{c}$ - anti-(M)-stable- $Z$

$\begin{array}{lrrr}\mathrm{C} & 2.278321 & 1.252900 & 0.047144 \\ \mathrm{C} & 3.323471 & 0.717314 & -0.616512 \\ \mathrm{C} & 1.033146 & 0.438457 & 0.207090 \\ \mathrm{~S} & 3.414697 & -0.941203 & -1.176124 \\ \mathrm{C} & 2.302669 & -1.663858 & -0.030319 \\ \mathrm{C} & 1.312921 & -0.981453 & 0.571547 \\ \mathrm{C} & -0.221833 & 0.935420 & 0.033141 \\ \mathrm{C} & -1.519494 & 0.217368 & -0.028186 \\ \mathrm{C} & -0.587550 & 2.414050 & -0.119202 \\ \mathrm{C} & -2.566167 & 1.112107 & 0.244776 \\ \mathrm{H} & 0.077777 & 3.052184 & 0.467700 \\ \mathrm{C} & -2.016130 & 2.494491 & 0.460405 \\ \mathrm{H} & -2.630979 & 3.269449 & -0.022324 \\ \mathrm{C} & -1.838796 & -1.100042 & -0.421926 \\ \mathrm{C} & -3.177092 & -1.518311 & -0.387389 \\ \mathrm{C} & -4.189341 & -0.623456 & -0.034841 \\ \mathrm{C} & -3.898187 & 0.706874 & 0.262293 \\ \mathrm{H} & -1.982974 & 2.728532 & 1.538312 \\ \mathrm{H} & -5.224799 & -0.973095 & -0.025544 \\ \mathrm{H} & -4.695046 & 1.418332 & 0.490329 \\ \mathrm{H} & -3.439956 & -2.539797 & -0.663303 \\ \mathrm{O} & -0.833323 & -1.878382 & -0.848229 \\ \mathrm{C} & -1.052473 & -3.242924 & -1.100109 \\ \mathrm{H} & -1.458539 & -3.760664 & -0.214807 \\ \mathrm{H} & -1.734279 & -3.398671 & -1.953329 \\ \mathrm{H} & -0.073925 & -3.674181 & -1.347367 \\ \mathrm{H} & 4.232626 & 1.295483 & -0.806868 \\ \mathrm{C} & 0.590227 & -1.593435 & 1.751105\end{array}$




$\begin{array}{lrrr}\mathrm{H} & -0.491996 & -1.416879 & 1.678062 \\ \mathrm{C} & 2.435011 & 2.641239 & 0.623899 \\ \mathrm{H} & 1.955108 & 2.718299 & 1.610806 \\ \mathrm{H} & 3.500025 & 2.882624 & 0.747655 \\ \mathrm{H} & 1.996605 & 3.416537 & -0.022634 \\ \mathrm{C} & -0.527048 & 2.858868 & -1.582472 \\ \mathrm{H} & 0.486404 & 2.727970 & -1.992363 \\ \mathrm{H} & -1.217534 & 2.267204 & -2.205113 \\ \mathrm{H} & -0.801345 & 3.920722 & -1.682582 \\ \mathrm{H} & 0.736534 & -2.684961 & 1.730945 \\ \mathrm{C} & 1.090213 & -1.031454 & 3.083004 \\ \mathrm{H} & 0.554328 & -1.491550 & 3.927712 \\ \mathrm{H} & 2.166321 & -1.222849 & 3.217642 \\ \mathrm{H} & 0.931203 & 0.056982 & 3.141625 \\ \mathrm{H} & 2.514675 & -2.710294 & 0.208577\end{array}$

motor 2c - TS1

$\begin{array}{llll}\text { C } & 2.330397 & -0.923212 & 0.756035\end{array}$

$\begin{array}{llll}\text { C } & 3.258068 & -1.105804 & -0.199114\end{array}$

$\begin{array}{llll}\text { C } & 1.093352 & -0.155423 & 0.399784\end{array}$

$\begin{array}{llll}\text { C } & 2.272945 & 1.130115 & -1.268233\end{array}$

$\begin{array}{llll}\text { C } & 1.420164 & 1.167005 & -0.225658\end{array}$

$\begin{array}{llll}\text { C } & -0.109110 & -0.779672 & 0.420403\end{array}$

$\begin{array}{llll}\text { C } & -1.437574 & -0.351422 & -0.097950\end{array}$

$\begin{array}{llll}\text { C } & -0.249890 & -2.294463 & 0.582537\end{array}$

$\begin{array}{lllll}\text { C } & -1.994172 & -1.432887 & -0.800675\end{array}$

$\mathrm{H} \quad 0.728416 \quad-2.790587 \quad 0.531863$

$\begin{array}{llll}\text { C } & -1.097276 & -2.637699 & -0.666563\end{array}$

$\mathrm{H} \quad-1.661551 \quad-3.576664 \quad-0.562806$

$\begin{array}{llll}\text { C } & -2.213402 & 0.802951 & 0.103939\end{array}$

$\begin{array}{llll}\text { C } & -3.449301 & 0.913263 & -0.554084\end{array}$

$\begin{array}{llll}\text { C } & -3.932365 & -0.138719 & -1.333613\end{array}$

C $\quad-3.224774 \quad-1.335833 \quad-1.442789$

$\mathrm{H} \quad-0.443130 \quad-2.733049-1.549912$

$\mathrm{H} \quad \begin{array}{llll}-4.900856 & -0.028257 & -1.827806\end{array}$

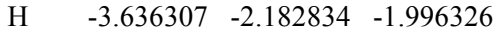

$\begin{array}{llll}\mathrm{H} & -4.057411 & 1.810212 & -0.435268\end{array}$

$\begin{array}{llll}\text { C } & 0.912927 & 2.490383 & 0.314501\end{array}$

$\begin{array}{llll}\mathrm{H} & 0.613344 & 2.335409 & 1.359785\end{array}$

$\begin{array}{llll}\mathrm{H} & -0.012678 & 2.765413 & -0.212355\end{array}$

$\begin{array}{llll}\text { C } & 2.529866 & -1.416098 & 2.162576\end{array}$

$\begin{array}{lllll}\mathrm{H} & 1.728806 & -2.100837 & 2.476748\end{array}$

$\begin{array}{llll}\text { C } & -0.963149 & -2.704447 & 1.872987\end{array}$

$\begin{array}{llll}\mathrm{H} & -1.061097 & -3.799665 & 1.930749\end{array}$

$\begin{array}{llll}\mathrm{H} & -0.412965 & -2.365033 & 2.763271\end{array}$

$\mathrm{H} \quad \begin{array}{llll}-1.975171 & -2.272427 & 1.925696\end{array}$

$\mathrm{H} \quad 2.546226 \quad 2.024224-1.834756$

$\begin{array}{llll}\text { O } & -1.759177 & 1.715129 & 0.979694\end{array}$

$\begin{array}{llll}\text { C } & -2.459243 & 2.921210 & 1.159630\end{array}$

$\mathrm{H} \quad-2.579703 \quad 3.471436 \quad 0.211355$

$\begin{array}{llll}\mathrm{H} & -3.453481 & 2.752997 & 1.607401\end{array}$

$\mathrm{H} \quad-1.861050 \quad 3.530594 \quad 1.848908$

$\begin{array}{llll}\mathrm{H} & 4.168526 & -1.690601 & -0.038529\end{array}$

$\begin{array}{llll}\mathrm{H} & 2.517482 & -0.569248 & 2.867909\end{array}$

$\begin{array}{llll}\text { S } & 3.146004 & -0.314353 & -1.766859\end{array}$

$\begin{array}{llll}\mathrm{C} & 1.901806 & 3.649003 & 0.227521\end{array}$

$\begin{array}{llll}\mathrm{H} & 2.133603 & 3.921960 & -0.813178\end{array}$

$\begin{array}{llll}\mathrm{H} & 1.477945 & 4.543984 & 0.708840\end{array}$

$\begin{array}{llll}\mathrm{H} & 2.850824 & 3.409196 & 0.732526\end{array}$

$\begin{array}{llll}\mathrm{H} & 3.491947 & -1.937127 & 2.270679\end{array}$

motor $2 \mathrm{c}-\mathrm{TS2}$

$\begin{array}{lllll}\text { C } & -0.403969 & -2.004433 & -0.764608\end{array}$

$\begin{array}{llll}\text { C } & -1.479558 & -1.018298 & -0.347381\end{array}$ 


$\begin{array}{rrrr}\mathrm{C} & -2.761616 & -1.404153 & -0.542936 \\ \mathrm{C} & -1.117062 & 0.372523 & 0.062204 \\ \mathrm{~S} & -4.216546 & -0.488855 & -0.306344 \\ \mathrm{C} & -3.547254 & 0.940955 & 0.403469 \\ \mathrm{C} & -2.236925 & 1.243486 & 0.531976 \\ \mathrm{C} & -1.935063 & 2.467651 & 1.365894 \\ \mathrm{C} & 0.153865 & 0.893847 & -0.035897 \\ \mathrm{C} & 1.468679 & 0.217360 & -0.145409 \\ \mathrm{C} & 0.482178 & 2.339285 & -0.455674 \\ \mathrm{C} & 2.251571 & 0.884290 & -1.098837 \\ \mathrm{H} & -0.409877 & 2.858462 & -0.829514 \\ \mathrm{C} & 1.462843 & 2.053491 & -1.627720 \\ \mathrm{H} & 2.093296 & 2.919274 & -1.880289 \\ \mathrm{C} & 2.063030 & -0.761816 & 0.670496 \\ \mathrm{C} & 3.383711 & -1.151891 & 0.404546 \\ \mathrm{C} & 4.112460 & -0.535065 & -0.616124 \\ \mathrm{C} & 3.563338 & 0.506421 & -1.365555 \\ \mathrm{H} & 0.899487 & 1.767922 & -2.532809 \\ \mathrm{H} & 5.141828 & -0.854630 & -0.796932 \\ \mathrm{H} & 4.157025 & 1.028474 & -2.119469 \\ \mathrm{C} & 1.194708 & 3.202655 & 0.591597 \\ \mathrm{H} & 0.585910 & 3.369752 & 1.488671 \\ \mathrm{H} & 2.137053 & 2.731212 & 0.912740 \\ \mathrm{H} & 1.443541 & 4.186733 & 0.164362 \\ \mathrm{H} & 3.862877 & -1.924691 & 1.006252 \\ \mathrm{H} & -4.309030 & 1.602670 & 0.825013 \\ \mathrm{H} & -2.985153 & -2.391218 & -0.949311 \\ \mathrm{H} & 0.202580 & -2.240938 & 0.117371 \\ \mathrm{H} & -2.833598 & 2.794108 & 1.907048 \\ \mathrm{H} & -1.154738 & 2.237722 & 2.106695 \\ \mathrm{H} & -1.585388 & 3.323894 & 0.774292 \\ \mathrm{O} & 1.325981 & -1.252494 & 1.688687 \\ \mathrm{C} & 1.886507 & -2.216081 & 2.547804 \\ \mathrm{H} & 1.119309 & -2.450697 & 3.296040 \\ \mathrm{H} & 2.151957 & -3.141352 & 2.008783 \\ \mathrm{H} & 2.781394 & -1.829207 & 3.063822 \\ \mathrm{H} & 0.269612 & -1.486269 & -1.463413 \\ \mathrm{C} & -0.851490 & -3.310474 & -1.406942 \\ \mathrm{H} & -1.413526 & -3.147628 & -2.340099 \\ \mathrm{H} & 0.036343 & -3.909780 & -1.659182 \\ \mathrm{H} & -1.473723 & -3.920684 & -0.733492 \\ & & & \end{array}$

motor $2 c-$ TS3

$\begin{array}{lrrr}\text { C } & -2.335569 & -0.526678 & 0.798569 \\ \mathrm{C} & -3.209597 & -0.767133 & -0.192226 \\ \mathrm{C} & -0.982368 & -0.021098 & 0.409120 \\ \mathrm{~S} & -2.819114 & -0.341036 & -1.859543 \\ \mathrm{C} & -1.853505 & 1.096723 & -1.525950 \\ \mathrm{C} & -1.132327 & 1.239765 & -0.395945 \\ \mathrm{C} & 0.132255 & -0.783104 & 0.530068 \\ \mathrm{C} & 1.526153 & -0.468516 & 0.095099 \\ \mathrm{C} & 0.187880 & -2.288910 & 0.866816 \\ \mathrm{C} & 2.134168 & -1.641100 & -0.377109 \\ \mathrm{H} & 0.663477 & -2.380978 & 1.861261 \\ \mathrm{C} & 1.209960 & -2.801998 & -0.174250 \\ \mathrm{H} & 0.682262 & -3.045272 & -1.113964 \\ \mathrm{C} & 2.306292 & 0.701995 & 0.179268 \\ \mathrm{C} & 3.598051 & 0.704359 & -0.370730 \\ \mathrm{C} & 4.137369 & -0.458069 & -0.923142 \\ \mathrm{C} & 3.422093 & -1.653274 & -0.905484 \\ \mathrm{H} & 1.732411 & -3.712185 & 0.155794 \\ \mathrm{H} & 5.147288 & -0.427676 & -1.339597 \\ \mathrm{H} & 3.863023 & -2.578757 & -1.282537 \\ \mathrm{H} & 4.205142 & 1.609180 & -0.341630 \\ \mathrm{O} & 1.798697 & 1.746405 & 0.851574\end{array}$




$\begin{array}{cccc}\mathrm{C} & 2.517843 & 2.952493 & 0.925020 \\ \mathrm{H} & 3.481906 & 2.822055 & 1.444606 \\ \mathrm{H} & 2.699668 & 3.384038 & -0.073750 \\ \mathrm{H} & 1.898486 & 3.648511 & 1.505006 \\ \mathrm{H} & -4.209449 & -1.180796 & -0.031079 \\ \mathrm{C} & -0.736389 & 2.623528 & 0.091057 \\ \mathrm{H} & -0.353743 & 2.528995 & 1.113236 \\ \mathrm{C} & -2.685846 & -0.636654 & 2.255902 \\ \mathrm{H} & -2.794189 & 0.370161 & 2.693173 \\ \mathrm{H} & -3.633726 & -1.174066 & 2.405715 \\ \mathrm{H} & -1.891566 & -1.142429 & 2.825683 \\ \mathrm{C} & -1.055696 & -3.169114 & 0.840861 \\ \mathrm{H} & -1.772942 & -2.948061 & 1.639241 \\ \mathrm{H} & -1.575829 & -3.097673 & -0.124776 \\ \mathrm{H} & -0.738006 & -4.215886 & 0.973294 \\ \mathrm{H} & -2.014483 & 1.917393 & -2.231084 \\ \mathrm{H} & 0.101373 & 3.009700 & -0.510242 \\ \mathrm{C} & -1.885433 & 3.631452 & 0.068253 \\ \mathrm{H} & -1.562693 & 4.591456 & 0.501366 \\ \mathrm{H} & -2.239528 & 3.832893 & -0.954094 \\ \mathrm{H} & -2.744624 & 3.266732 & 0.653282\end{array}$

motor 2c - (P)-unstable- $E$

$\begin{array}{lrrr}\mathrm{C} & 3.383646 & 0.297370 & 0.421859 \\ \mathrm{C} & 0.971999 & 0.195326 & -0.239090 \\ \mathrm{~S} & 3.945468 & -1.014526 & -0.561426 \\ \mathrm{C} & 2.445964 & -1.537798 & -1.261096 \\ \mathrm{C} & 1.214522 & -1.006608 & -1.072719 \\ \mathrm{C} & -0.272204 & 0.794056 & -0.186852 \\ \mathrm{C} & -1.585700 & 0.140645 & -0.165642 \\ \mathrm{C} & -0.552795 & 2.303814 & -0.197508 \\ \mathrm{C} & -2.600466 & 1.057704 & -0.480760 \\ \mathrm{H} & -0.616304 & 2.698324 & 0.834289 \\ \mathrm{C} & -1.979295 & 2.384778 & -0.805816 \\ \mathrm{H} & -1.905069 & 2.511673 & -1.900821 \\ \mathrm{C} & -1.944325 & -1.123825 & 0.351831 \\ \mathrm{C} & -3.299618 & -1.478657 & 0.402003 \\ \mathrm{C} & -4.284586 & -0.571966 & 0.001338 \\ \mathrm{C} & -3.947185 & 0.714257 & -0.422674 \\ \mathrm{H} & -2.560326 & 3.235724 & -0.420909 \\ \mathrm{H} & -5.333966 & -0.872097 & 0.057172 \\ \mathrm{H} & -4.721924 & 1.439033 & -0.683185 \\ \mathrm{H} & -3.599679 & -2.457182 & 0.777872 \\ \mathrm{C} & 2.117190 & 0.767104 & 0.510852 \\ \mathrm{C} & 0.136190 & -1.534848 & -1.989773 \\ \mathrm{H} & -0.638142 & -2.102268 & -1.461157 \\ \mathrm{H} & -0.362594 & -0.701845 & -2.509389 \\ \mathrm{C} & 1.866499 & 1.718600 & 1.671741 \\ \mathrm{H} & 1.320108 & 2.616966 & 1.363074 \\ \mathrm{H} & 2.840246 & 2.076335 & 2.039330 \\ \mathrm{H} & 4.164581 & 0.692977 & 1.077530 \\ \mathrm{C} & 0.431029 & 3.143371 & -1.008971 \\ \mathrm{H} & 0.107911 & 4.196338 & -1.029265 \\ \mathrm{H} & 1.455589 & 3.119331 & -0.613289 \\ \mathrm{H} & 0.472662 & 2.781789 & -2.048848 \\ \mathrm{O} & -0.949400 & -1.908258 & 0.810504 \\ \mathrm{C} & -1.246074 & -3.186296 & 1.315066 \\ \mathrm{H} & -1.903967 & -3.136692 & 2.199452 \\ \mathrm{H} & -1.717180 & -3.827966 & 0.551089 \\ \mathrm{H} & -0.289166 & -3.633838 & 1.611245 \\ \mathrm{H} & 2.590147 & -2.343704 & -1.985926 \\ \mathrm{H} & 0.572089 & -2.197551 & -2.749675 \\ \mathrm{C} & 1.115357 & 1.050377 & 2.824969 \\ \mathrm{H} & 1.677790 & 0.187022 & 3.213475 \\ \mathrm{H} & 0.969667 & 1.762306 & 3.651897\end{array}$


motor 2c - syn-(M)-stable- $E$

\begin{tabular}{|c|c|c|c|}
\hline $\mathrm{C}$ & -2.175215 & -1.624050 & -1 \\
\hline$C$ & -1.073047 & -0.138754 & 0.072955 \\
\hline & -3.395766 & -0.428088 & -1.910597 \\
\hline & -3.431273 & 0.433198 & -0.383979 \\
\hline 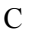 & -2.388165 & 0.462802 & 0.463501 \\
\hline & 0.056395 & 0.610208 & 0.16 \\
\hline 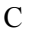 & 1.458640 & 0.297 & -0.2 \\
\hline C & 0.038462 & 2.139518 & 0.232087 \\
\hline C & 1.952920 & 1.369207 & -0.9 \\
\hline I & -0.979248 & 2.530624 & 0.096229 \\
\hline C & 0.920469 & 2.470285 & -1.002 \\
\hline $\mathrm{H}$ & 1.373426 & 3.47 & -0.9 \\
\hline 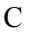 & 2.340363 & -0.710145 & 0.2 \\
\hline C & 3.639734 & -0.735589 & -0.3 \\
\hline $\mathrm{C}$ & 4.070 & 0.279141 & -1.17 \\
\hline $\mathrm{C}$ & 3.2 & 1.36 & -1.4 \\
\hline $\mathrm{H}$ & 0.31 & 2.416463 & -1.9 \\
\hline $\mathrm{H}$ & 5.08 & 0.239714 & -1.5 \\
\hline $\mathrm{H}$ & 3.615246 & 2.193 & -2.090511 \\
\hline $\mathrm{H}$ & 4.3 & -1.5 & -0.0 \\
\hline $\mathrm{C}$ & -1.207844 & -1.477025 & -0.5 \\
\hline $\mathrm{C}$ & -2.56 & 1.002254 & \\
\hline $\mathrm{H}$ & -1.854062 & 1.825051 & 2.0 \\
\hline $\mathrm{C}$ & -0.321317 & -2.656509 & -0.2 \\
\hline $\mathrm{H}$ & -0.21 & -2.810286 & 0.7 \\
\hline $\mathrm{H}$ & -0.745827 & -3.568158 & \\
\hline $\mathrm{H}$ & 0.686553 & -2.517973 & -0.701862 \\
\hline $\mathrm{H}$ & -2.27 & -2.555202 & -2.0 \\
\hline $\mathrm{C}$ & 0.645617 & 2.719764 & 1.51 \\
\hline $\mathrm{H}$ & 0.622843 & 3.820351 & 1.4 \\
\hline $\mathrm{H}$ & 0.098733 & 2.391478 & 2.407686 \\
\hline $\mathrm{H}$ & 1.695023 & 2.407 & 1.6 \\
\hline $\mathrm{O}$ & 1.905915 & -1.556988 & 1.163364 \\
\hline $\mathrm{C}$ & 2.712600 & -2.644208 & 1.543086 \\
\hline $\mathrm{H}$ & 2.950176 & -3.298000 & 0.686718 \\
\hline $\mathrm{H}$ & 3.652952 & -2.309783 & 2.01278 \\
\hline $\mathrm{H}$ & 2.138181 & -3.220006 & 2.279665 \\
\hline $\mathrm{H}$ & -4.395551 & 0.887575 & -0.13781 \\
\hline $\mathrm{H}$ & -3.569967 & 1.431161 & 1.96232 \\
\hline $\mathrm{C}$ & -2.351339 & -0.070612 & 2.93552 \\
\hline $\mathrm{H}$ & -2.476364 & 0.355253 & 3.94290 \\
\hline $\mathrm{H}$ & -3.076118 & -0.891985 & 2.820 \\
\hline & -1339416 & -0.501136 & 287 \\
\hline
\end{tabular}

motor 2c - anti-(M)-stable- $E$

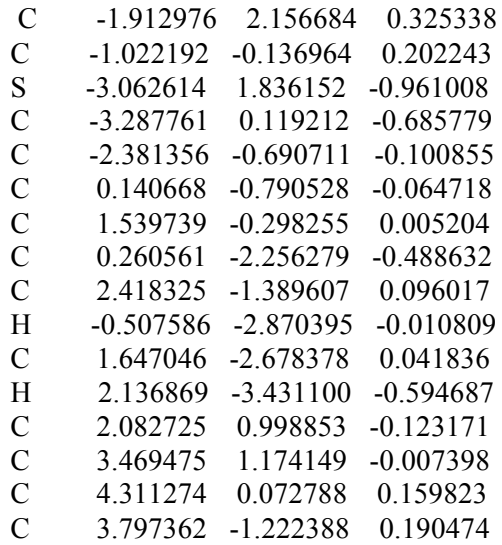




$\begin{array}{lrrr}\mathrm{H} & 1.557722 & -3.114960 & 1.051228 \\ \mathrm{H} & 5.388859 & 0.237148 & 0.237092 \\ \mathrm{H} & 4.460508 & -2.086634 & 0.272215 \\ \mathrm{H} & 3.904522 & 2.171125 & -0.080156 \\ \mathrm{C} & -1.082415 & 1.221292 & 0.817593 \\ \mathrm{C} & -2.817311 & -2.089951 & 0.290275 \\ \mathrm{C} & -0.312302 & 1.479023 & 2.085415 \\ \mathrm{H} & 0.732920 & 1.147767 & 2.023152 \\ \mathrm{H} & -0.325429 & 2.546639 & 2.347947 \\ \mathrm{H} & -0.772872 & 0.920426 & 2.917586 \\ \mathrm{H} & -1.967549 & 3.166311 & 0.742940 \\ \mathrm{C} & 0.137579 & -2.418896 & -2.005285 \\ \mathrm{H} & 0.224840 & -3.478512 & -2.292867 \\ \mathrm{H} & -0.834036 & -2.045159 & -2.364149 \\ \mathrm{H} & 0.927518 & -1.857686 & -2.530150 \\ \mathrm{O} & 1.233923 & 2.001774 & -0.391124 \\ \mathrm{C} & 1.681599 & 3.333300 & -0.372501 \\ \mathrm{H} & 2.138248 & 3.596408 & 0.596610 \\ \mathrm{H} & 2.406489 & 3.534246 & -1.179502 \\ \mathrm{H} & 0.794557 & 3.959867 & -0.531558 \\ \mathrm{H} & -4.275680 & -0.248964 & -0.978496 \\ \mathrm{H} & -2.346979 & -2.844450 & -0.358964 \\ \mathrm{C} & -2.538132 & -2.418622 & 1.755180 \\ \mathrm{H} & -2.894629 & -3.430148 & 2.001992 \\ \mathrm{H} & -1.462361 & -2.375059 & 1.986116 \\ \mathrm{H} & -3.048656 & -1.705633 & 2.421501 \\ \mathrm{H} & -3.898303 & -2.172188 & 0.101710\end{array}$

motor $2 c$ - TS4

$\begin{array}{lrrr}\mathrm{C} & 2.387960 & 0.077683 & -0.341954 \\ \mathrm{C} & 3.142687 & -0.134197 & 0.749788 \\ \mathrm{C} & 0.956998 & -0.367456 & -0.280921 \\ \mathrm{C} & 1.475219 & -2.163462 & 1.237872 \\ \mathrm{C} & 0.842442 & -1.810071 & 0.102495 \\ \mathrm{C} & -0.027919 & 0.561466 & -0.273011 \\ \mathrm{C} & -1.467727 & 0.436524 & 0.078367 \\ \mathrm{C} & 0.245989 & 2.064170 & -0.193027 \\ \mathrm{C} & -1.808552 & 1.526101 & 0.896007 \\ \mathrm{H} & 1.306238 & 2.262487 & 0.013347 \\ \mathrm{C} & -0.630100 & 2.458247 & 1.018925 \\ \mathrm{H} & -0.926169 & 3.518247 & 1.014222 \\ \mathrm{C} & -2.473464 & -0.458018 & -0.325077 \\ \mathrm{C} & -3.759490 & -0.325160 & 0.222817 \\ \mathrm{C} & -4.048917 & 0.717723 & 1.104473 \\ \mathrm{C} & -3.084470 & 1.670043 & 1.432775 \\ \mathrm{H} & -0.084276 & 2.264820 & 1.958138 \\ \mathrm{H} & -5.059714 & 0.801185 & 1.511605 \\ \mathrm{H} & -3.328985 & 2.517038 & 2.077796 \\ \mathrm{H} & -4.550721 & -1.020663 & -0.057690 \\ \mathrm{C} & 0.184285 & -2.867423 & -0.753252 \\ \mathrm{H} & 0.042420 & -2.510809 & -1.780542 \\ \mathrm{H} & 0.805489 & -3.774863 & -0.779701 \\ \mathrm{H} & -0.803967 & -3.148388 & -0.364217 \\ \mathrm{C} & 2.917665 & 0.682903 & -1.620105 \\ \mathrm{H} & 2.214905 & 1.457758 & -1.962977 \\ \mathrm{C} & -0.176940 & 2.822248 & -1.453561 \\ \mathrm{H} & 0.019381 & 3.899593 & -1.339358 \\ \mathrm{H} & 0.368502 & 2.468260 & -2.341164 \\ \mathrm{H} & -1.253160 & 2.693359 & -1.651827 \\ \mathrm{H} & 1.437041 & -3.183841 & 1.632010 \\ \mathrm{O} & -2.158839 & -1.360201 & -1.269002 \\ \mathrm{C} & -3.126315 & -2.281705 & -1.706706 \\ \mathrm{H} & -3.493355 & -2.916617 & -0.882923 \\ \mathrm{H} & -3.983747 & -1.776901 & -2.183178 \\ \mathrm{H} & -2.635040 & -2.919112 & -2.452837\end{array}$




$\begin{array}{lrrr}\mathrm{H} & 4.179125 & 0.198372 & 0.845337 \\ \mathrm{H} & 2.873654 & -0.101555 & -2.396382 \\ \mathrm{C} & 4.323826 & 1.259349 & -1.551797 \\ \mathrm{H} & 4.392947 & 2.062307 & -0.801028 \\ \mathrm{H} & 5.069164 & 0.490764 & -1.294564 \\ \mathrm{H} & 4.609525 & 1.685961 & -2.524686 \\ \mathrm{~S} & 2.552025 & -1.081223 & 2.115499\end{array}$

motor 2c - TS5

$\begin{array}{llll}\text { C } & 0.153939 & -2.399776 & -0.798206\end{array}$

$\begin{array}{llll}\text { C } & -1.073404 & -1.553238 & -0.566173\end{array}$

$\begin{array}{llll}\text { C } & -2.239929 & -2.130520 & -0.938365\end{array}$

$\begin{array}{llll}\text { C } & -0.989979 & -0.122329 & -0.160937\end{array}$

$\begin{array}{llll}\mathrm{S} & -3.830005 & -1.433063 & -0.942124\end{array}$

$\begin{array}{llll}\text { C } & -3.496842 & 0.048633 & -0.106427\end{array}$

$\begin{array}{llll}\text { C } & -2.280293 & 0.542681 & 0.205859\end{array}$

$\begin{array}{llll}\text { C } & -2.228030 & 1.758796 & 1.118068\end{array}$

$\begin{array}{llll}\text { C } & 0.176028 & 0.606118 & -0.183157\end{array}$

$\begin{array}{llll}\text { C } & 1.587958 & 0.155480 & -0.219512\end{array}$

$\begin{array}{llll}\text { C } & 0.278780 & 2.082852 & -0.605262\end{array}$

$\begin{array}{llll}\text { C } & 2.292837 & 0.932247 & -1.150134\end{array}$

$\begin{array}{llll}\mathrm{H} & -0.668760 & 2.443712 & -1.027292\end{array}$

$\begin{array}{llll}\text { C } & 1.349676 & 1.957241 & -1.725813\end{array}$

$\begin{array}{llll}\mathrm{H} & 1.843947 & 2.913441 & -1.953786\end{array}$

$\begin{array}{llll}\text { C } & 2.297525 & -0.700217 & 0.641707\end{array}$

$\begin{array}{llll}\text { C } & 3.673539 & -0.876289 & 0.434608\end{array}$

$\begin{array}{llll}\text { C } & 4.335436 & -0.164668 & -0.569926\end{array}$

$\begin{array}{llll}\text { C } & 3.658378 & 0.767248 & -1.358140\end{array}$

$\begin{array}{llll}\mathrm{H} & 0.884070 & 1.584023 & -2.653964\end{array}$

$\begin{array}{llll}\mathrm{H} & 5.408790 & -0.318581 & -0.706047\end{array}$

$\begin{array}{llll}\mathrm{H} & 4.192626 & 1.370415 & -2.095879\end{array}$

$\begin{array}{llll}\text { C } & 0.780749 & 3.056629 & 0.466012\end{array}$

$\begin{array}{llll}\mathrm{H} & 0.099794 & 3.126523 & 1.323882\end{array}$

$\begin{array}{llll}\mathrm{H} & 1.766817 & 2.747424 & 0.847595\end{array}$

$\begin{array}{llll}\mathrm{H} & 0.890249 & 4.065870 & 0.038892\end{array}$

$\begin{array}{llll}\mathrm{H} & 4.246519 & -1.551264 & 1.070844\end{array}$

$\begin{array}{llll}\mathrm{H} & -4.410416 & 0.551847 & 0.215636\end{array}$

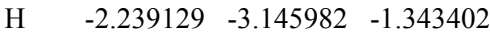

$\begin{array}{llll}\mathrm{H} & 0.694988 & -2.593110 & 0.134310\end{array}$

$\begin{array}{llll}\mathrm{H} & -1.397362 & 1.592262 & 1.822107\end{array}$

$\begin{array}{llll}\mathrm{H} & -1.947625 & 2.652072 & 0.541241\end{array}$

$\begin{array}{llll}\text { O } & 1.609828 & -1.280878 & 1.646559\end{array}$

$\begin{array}{llll}\text { C } & 2.283613 & -2.125126 & 2.548112\end{array}$

$\begin{array}{llll}\mathrm{H} & 1.536316 & -2.460152 & 3.278243\end{array}$

$\begin{array}{llll}\mathrm{H} & 2.707634 & -3.009732 & 2.043405\end{array}$

$\begin{array}{llll}\mathrm{H} & 3.089588 & -1.593786 & 3.082054\end{array}$

$\begin{array}{lllll}\mathrm{H} & 0.847314 & -1.907616 & -1.494889\end{array}$

$\begin{array}{llll}\text { C } & -3.489322 & 2.085417 & 1.908398\end{array}$

$\begin{array}{llll}\mathrm{H} & -3.285329 & 2.918997 & 2.597181\end{array}$

$\begin{array}{llll}\mathrm{H} & -4.322573 & 2.400827 & 1.261730\end{array}$

$\begin{array}{llll}\mathrm{H} & -3.829554 & 1.228143 & 2.510338\end{array}$

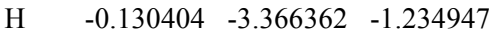

motor 2c - TS6

$\begin{array}{llll}\text { C } & -2.314192 & -0.112315 & -0.386476\end{array}$

$\begin{array}{llll}\text { C } & -3.043600 & -0.121054 & 0.741583\end{array}$

$\begin{array}{llll}\text { C } & -0.848180 & -0.377156 & -0.236041\end{array}$

$\begin{array}{llll}\mathrm{S} & -2.317710 & -0.614202 & 2.277033\end{array}$

$\begin{array}{llll}\text { C } & -1.203666 & -1.825904 & 1.641384\end{array}$

$\begin{array}{llll}\text { C } & -0.658132 & -1.717567 & 0.413609\end{array}$

$\begin{array}{llll}\text { C } & 0.080349 & 0.598601 & -0.387973\end{array}$

$\begin{array}{llll}\text { C } & 1.550325 & 0.536547 & -0.127671\end{array}$

$\begin{array}{llll}\text { C } & -0.189767 & 2.102277 & -0.615861\end{array}$

$\begin{array}{llll}\text { C } & 1.990762 & 1.787656 & 0.330567\end{array}$ 


$\begin{array}{lrrr}\mathrm{H} & 0.110014 & 2.321793 & -1.658091 \\ \mathrm{C} & 0.855817 & 2.762679 & 0.308315 \\ \mathrm{H} & 0.426309 & 2.879377 & 1.319550 \\ \mathrm{C} & 2.512347 & -0.472725 & -0.335097 \\ \mathrm{C} & 3.841838 & -0.244281 & 0.053397 \\ \mathrm{C} & 4.228576 & 0.988013 & 0.581202 \\ \mathrm{C} & 3.310123 & 2.027173 & 0.704430 \\ \mathrm{H} & 1.157055 & 3.762757 & -0.037629 \\ \mathrm{H} & 5.272643 & 1.138740 & 0.866656 \\ \mathrm{H} & 3.616608 & 3.010134 & 1.069147 \\ \mathrm{H} & 4.592869 & -1.022602 & -0.080563 \\ \mathrm{O} & 2.121115 & -1.593735 & -0.959075 \\ \mathrm{C} & 3.049055 & -2.623014 & -1.196660 \\ \mathrm{H} & 3.875461 & -2.284963 & -1.844152 \\ \mathrm{H} & 3.465152 & -3.026099 & -0.258168 \\ \mathrm{H} & 2.504498 & -3.423018 & -1.714194 \\ \mathrm{H} & -1.135911 & -2.745018 & 2.232283 \\ \mathrm{C} & -2.883186 & 0.031483 & -1.776600 \\ \mathrm{H} & -2.758737 & -0.944146 & -2.280125 \\ \mathrm{H} & -2.245379 & 0.724411 & -2.350076 \\ \mathrm{C} & -0.160449 & -2.956315 & -0.299229 \\ \mathrm{H} & 0.097189 & -2.740453 & -1.340907 \\ \mathrm{H} & 0.726445 & -3.384479 & 0.189386 \\ \mathrm{H} & -0.946864 & -3.726443 & -0.290644 \\ \mathrm{C} & -1.557285 & 2.727677 & -0.366284 \\ \mathrm{H} & -2.321633 & 2.431546 & -1.093077 \\ \mathrm{H} & -1.926324 & 2.497692 & 0.643104 \\ \mathrm{H} & -1.450575 & 3.821788 & -0.442146 \\ \mathrm{H} & -4.112613 & 0.101381 & 0.785843 \\ \mathrm{C} & -4.339411 & 0.463920 & -1.853533 \\ \mathrm{H} & -4.658405 & 0.558553 & -2.902053 \\ \mathrm{H} & -5.004867 & -0.266609 & -1.367852 \\ \mathrm{H} & -4.496431 & 1.438590 & -1.364601\end{array}$

